\title{
Die Rolle von Wissenschaft und Innovation in der Flughafenumfeldregion
}

Herausforderungen und Perspektiven

Dana Mietzner, Frank Hartmann, Klaus-Martin Melzer, Markus Vossel, Franziska Bautz, Henning Wiechers unter Mitwirkung von Martina Konieczny, Manuel Haberland und Peter Kessel

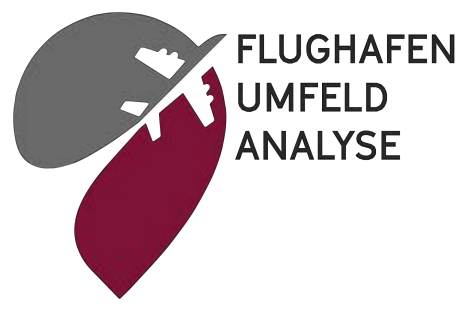

Technische Hochschule Wildau

Technologietransfer- und Weiterbildungszentrum an der Technischen Hochschule Wildau e.V.

Hochschulring 1 I 15745 Wildau

Dezember 2020

Kontakt: Markus Vossel I email: markus.vossel@th-wildau.de I 03375 - 508673 
Inhaltsverzeichnis

DARSTELLUNGSVERZEICHNIS

ABKÜRZUNGSVERZEICHNIS

1 ZIELSETZUNG

2 METHODISCHES VORGEHEN

3 ERGEBNISSE

3.1 Festlegung der Gebietskulisse der FUR 9

3.2 Charakterisierung der Städte und Gemeinden 13

3.3 Identifikation des Wissenschafts- und Technologiepotenzials 18

3.4 Ermittlung der Perspektive ausgewählter Akteur/innen $\quad 27$

3.5 Good Practice Beispiele $\quad 31$

$\begin{array}{lll}3.6 & \text { Handlungsansätze } & 35\end{array}$

4 DAS DIGITALE FORUM „DIE ROLLE VON WISSENSCHAFT UND INNOVATION IN DER FLUGHAFENUMFELDREGION - HERAUSFORDERUNGEN UND PERSPEKTIVEN“

$\begin{array}{ll}\text { LITERATURVERZEICHNIS } & 45\end{array}$

$\begin{array}{ll}\text { Anlagen } & 48\end{array}$

$\begin{array}{ll}\text { ANLAGE } 1 \text { GEMEINDESTECKBRIEFE } & 49\end{array}$

$\begin{array}{ll}\text { ANLAGE } 2 \text { GOOD PRACTICE BEISPIELE } & 68\end{array}$

$\begin{array}{ll}\text { ANLAGE } 3 \text { PROGRAMM DIGITALES FORUM } & 73\end{array}$ 


\section{Darstellungsverzeichnis}

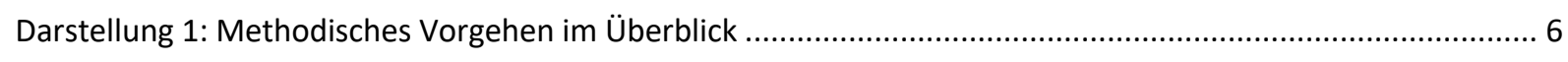

Darstellung 2: Flughafenumfeldregion im Gemeinsamen Strukturkonzept aus dem Jahr 2007 ........................... 10

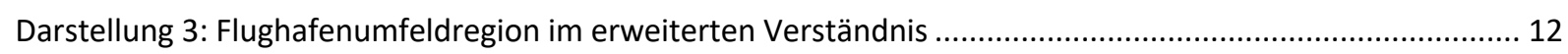

Darstellung 4: Flughafenumfeldregion im erweiterten Verständnis

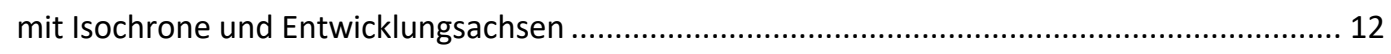

Darstellung 5: Merkmale und Indikatoren für die Charakterisierung der Städte und Gemeinden ..................... 13

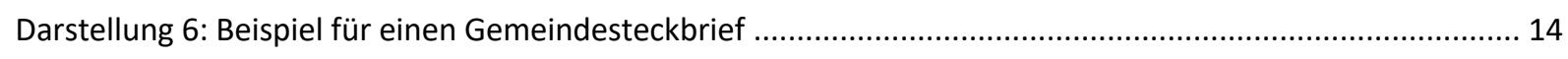

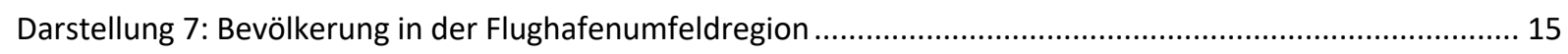

Darstellung 8: Unternehmensbesatz und Unternehmensansiedlung in der Flughafenumfeldregion ................. 15

Darstellung 9: Fachkräftebedarf in ausgewählten Städten/Gemeinden der Flughafenumfeldregion ................. 16

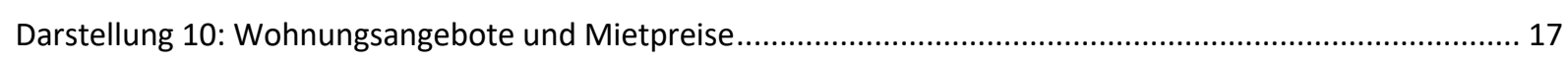

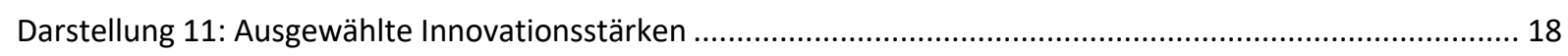

Darstellung 12: Technologiezentren im Berliner Teil der Flughafenumfeldregion (Standort Adlershof) ............. 19

Darstellung 13: Forschungseinrichtungen im Berliner Teil der Flughafenumfeldregion (Standort Adlershof) .... 19

Darstellung 14: Technologiestandorte in Berliner Teil der Flughafenumfeldregion (Oberschöneweide) ............ 20

Darstellung 15: Forschungseinrichtungen und Technologiezentren im Brandenburger Teil der Flughafenumfeldregion

Darstellung 16: Innovative, technologieorientierte Unternehmen im Brandenburger Teil der

Flughafenumfeldregion nach Clustern und Patentaktivitäten (Stand 04.09.2020) ................... 23

Darstellung 17: Innovative, technologieorientierte Unternehmen nach Clustern sowie Städten/Gemeinden ... 24

Darstellung 18: Herausforderungen für nachhaltige Flughafenregionen .......................................................... 33

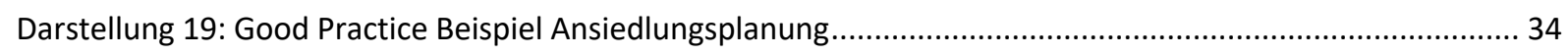

Darstellung 20: Good Practice Beispiel Innovationsstandort .................................................................... 34

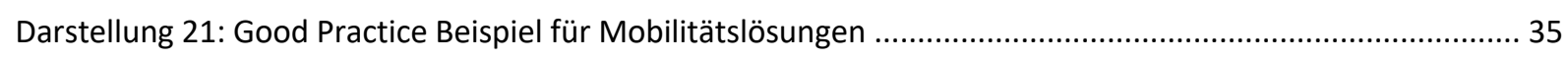

Darstellung 22: Adressierte Akteure/innen für die Operationalisierung und

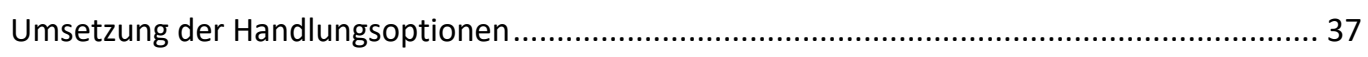

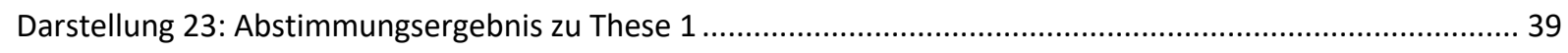

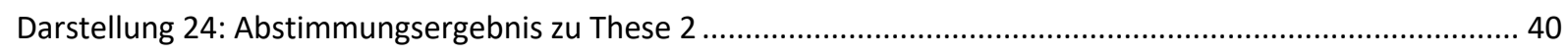

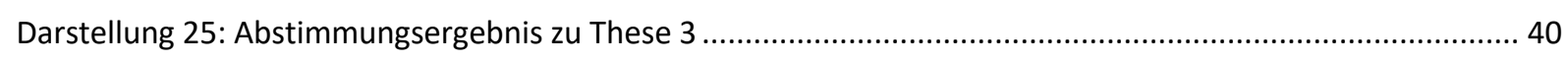

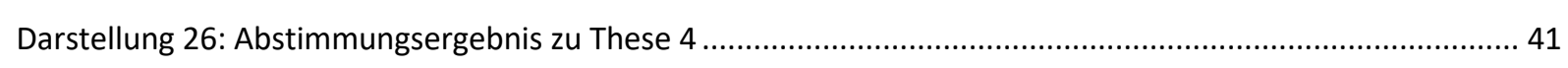

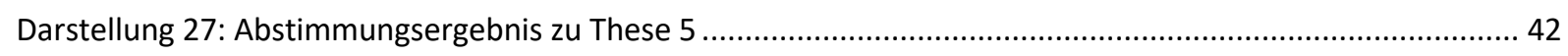

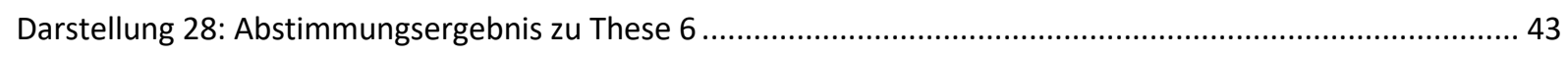

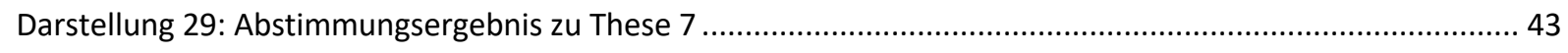




\section{Abkürzungsverzeichnis}

$\begin{array}{ll}\text { Auto ID } & \text { Automatische Identifikation und Datenerfassung } \\ \text { BER } & \text { Flughafen Berlin Brandenburg } \\ \text { BIF } & \text { Brandenburger Innovationsfachkräfte } \\ \text { EFRE } & \text { Europäischer Fonds für Regionale Entwicklung } \\ \text { ESF } & \text { Europäischer Sozialfonds } \\ \text { FH } & \text { Fachhochschule } \\ \text { GSK } & \text { Gemeinsames Strukturkonzept } \\ \text { IAU } & \text { île-de-France - Paris Region Urban Planning and Development Agency } \\ \text { IOT } & \text { Internet of Things } \\ \text { KAG DF } & \text { Kommunale Arbeitsgemeinschaft Dialogforum } \\ \text { KMU } & \text { Kleine und Mittlere Unternehmen } \\ \text { KW } & \text { Königs Wusterhausen } \\ \text { MWAE } & \text { Ministerium für Wirtschaft, Arbeit und Energie des Landes Brandenburg } \\ \text { MWFK } & \text { Ministerium für Wissenschaft, Forschung Kultur des Landes Brandenburg } \\ \text { RFID } & \text { Radio-Frequency Identification } \\ \text { RWK } & \text { Regionaler Wachstumskern } \\ \text { TH } & \text { Technische Hochschule } \\ \text { USP } & \text { Unique Selling Proposition } \\ \text { WFG } & \text { Wirtschaftsförderung Dahme-Spreewald } \\ & \end{array}$




\section{$1 \quad$ Zielsetzung}

Mit der Eröffnung des Flughafens BER im Oktober 2020, dem Bau der Gigafactory von Tesla in Grünheide und der Microvast-Batteriefabrik in Ludwigsfelde beginnend im Sommer 2020 sind deutlich Signale für den Beginn einer neuen Phase der Entwicklung der Flughafenumfeldregion erkennbar.

Konzeptionell begleitet wird dieser Prozess zunächst durch die Fortschreibung des Gemeinsamen Strukturkonzeptes der Flughafenregion. Sie wurde von der Kommunalen Arbeitsgemeinschaft Dialogforum Airport Berlin Brandenburg (KAG DF) im Jahr 2019 beauftragt und soll voraussichtlich im April 2021 abgeschlossen und bestätigt werden (vgl. Dialogforum-BER, 2020). Hinzu kommen die Grundlagenermittlung Verkehr Flughafenregion BER, die mit dem Bericht vom August 2019 abgeschlossen wurde, sowie der Dachmarkenprozess „Entwicklungsstrategie und Dachmarkenkonzeption für die Technologie- und Wissenschaftsregion Zeuthen - Wildau - Königs Wusterhausen“, der im Jahr 2019 begann und dessen Ergebnisse im Sommer 2020 veröffentlicht wurden. Schließlich beauftragte das Ministerium für Wissenschaft, Forschung und Kultur des Landes Brandenburg die Technische Hochschule Wildau Ende 2019 mit der Analyse der Flughafenumfeldregion BER, für die die Frage nach der Beschaffenheit und Ansätzen einer Stärkung des Wissenschafts- und Technologiepotenzials der Region leitend war. Mit der Analyse sollten die Perspektiven verschiedener Akteur/innen, insbesondere aus den Bereichen Wirtschaftspolitik und Wirtschaftsförderung sowie Wissenschaft, auf die Region erhoben, aktuelle Projekte gesichtet und der Stand von Entwicklungsvorhaben, die mit der Entwicklung der Region in Zusammenhang stehen, ermittelt werden.

Konkrete Aufgaben hierbei waren (1) die Charakterisierung der Städte und Gemeinden der Flughafenumfeldregion, (2) die Identifizierung der Wissenschafts- und Innovationspotenziale der Region, (3) die Befragung von Regionalakteur/innen zum Status quo sowie (4) die Ermittlung von Good Practices in anderen Flughafenumfeldregionen. Basierend auf den damit gewonnenen Ergebnissen waren erste Handlungsansätze abzuleiten, die auf einem Dialogforum vorgestellt, diskutiert und konkretisiert werden sollten.

Obwohl es sich bei der Flughafenumfeldregion BER um eine komplexe, Bundesländer übergreifende Region handelt, lag der Fokus der Analyse auf dem Brandenburger Teil der Region. Was den Berliner Teil betrifft, wurden vor allem die Wissenschafts- und Technologiepotenziale an den Standorten Adlershof und Oberschöneweide berücksichtigt.

Die Technische Hochschule Wildau versteht sich selbst als Akteurin der Flughafenumfeldregion, verfügt über umfangreiche Erfahrungen in der Interaktion mit Regionalakteur/innen und damit gute Vo- 
raussetzungen, um eine Analyse durchzuführen, welche die Arbeiten zum GSK und zur Verkehrssituation ergänzt. Mit der Analyse war ebenfalls beabsichtigt, sich abzeichnende neue Kooperationsbeziehungen mit ansiedlungsinteressierten Wissenschaftseinrichtungen zu vertiefen.

Die Analyse fand zum größten Teil im Jahr der Eröffnung des BER und damit unter den erschwerenden Bedingungen der Covid-19-Pandemie statt und umfasste einen Zeitraum von etwa 10 Monaten. Ihren vorläufigen Abschluss fand die Analyse mit dem digitalen Forum „Die Rolle von Wissenschaft und Innovation in der Flughafenumfeldregion - Herausforderungen und Perspektiven" am 03. Dezember 2020.

\section{Methodisches Vorgehen}

Das methodische Vorgehen beruhte auf einem qualitativen Untersuchungsansatz. Er umfasste umfangreiche Recherchen zu den Städten und Gemeinden der Flughafenumfeldregion, die Identifizierung innovativer, technologieorientierter Unternehmen durch die Analyse entsprechender Förderprogramme und Wettbewerbe, die Ermittlung von Good Practices durch die Auswertung der Aktivitäten der Sustainable Airport Areas Initiative, die in den Guidelines for Decision Makers aus dem Jahr 2018 zusammenfassend dokumentiert sind sowie die Konzipierung und Durchführung von Experteninterviews mit Regionalakteur/innen. Das methodische Vorgehen im Überblick zeigt die Darstellung 1.

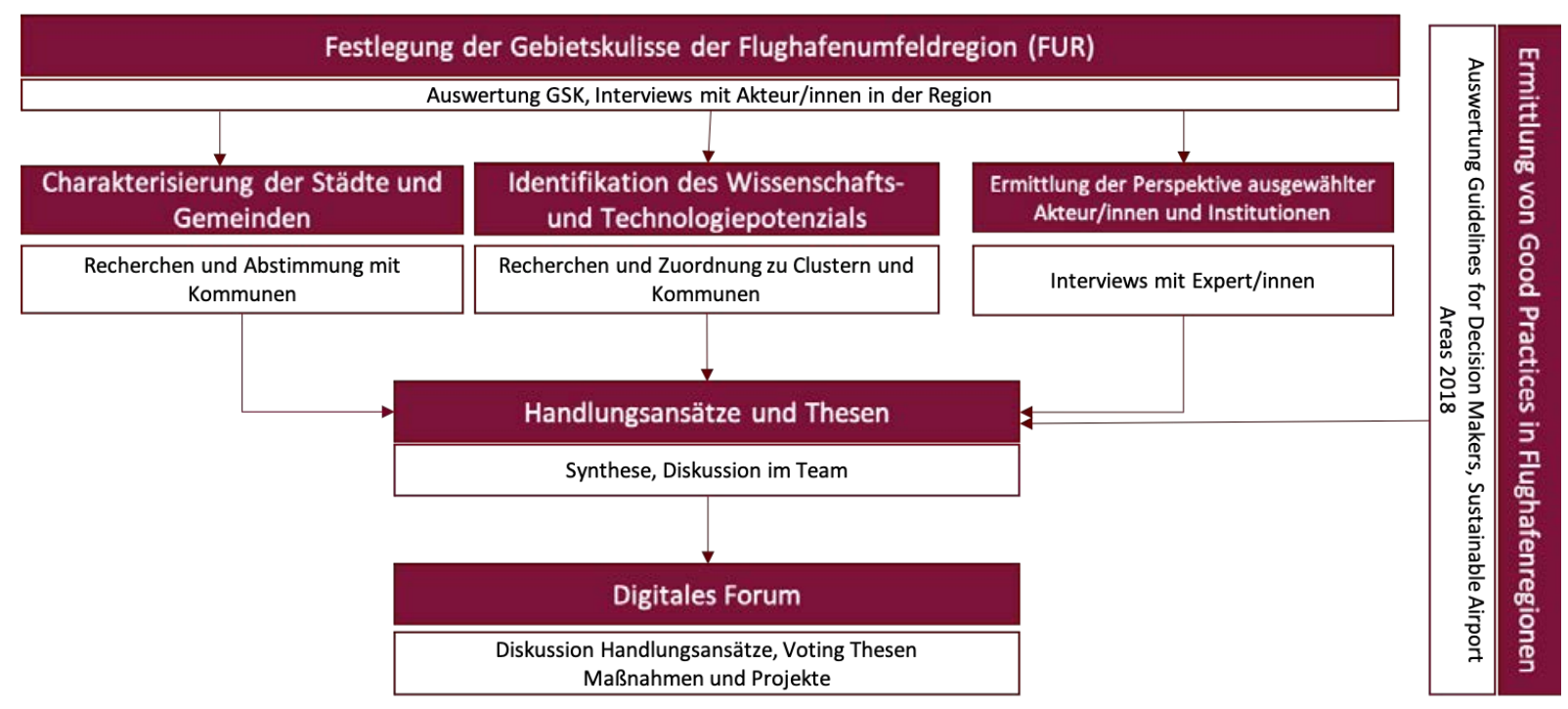

Darstellung 1: Methodisches Vorgehen im Überblick 


\section{Festlegung der Gebietskulisse der FUR}

Am Anfang der Analyse stand die Auseinandersetzung mit der Definition der Gebietskulisse der Flughafenumfeldregion. Hierfür wurde zunächst auf die Definition im Gemeinsamen Strukturkonzept aus dem Jahr 2007 zurückgegriffen (vgl. GSK, 2007:13). Erste Diskussionen im Bearbeitungsteam zeigten jedoch recht schnell, dass die Gebietskulisse der Flughafenumfeldregion aufgrund der Entwicklungen in den vergangenen Jahren einer Überarbeitung bedarf. Sie wurde daher im Sinne einer Arbeitsdefinition erweitert, die im Ergebnis der Interviews von Regionalakteur/innen als geeignet bestätigt wurde.

\section{Charakterisierung der Städte und Gemeinden}

Die Charakterisierung der Städte und Gemeinden der Flughafenumfeldregion erfolgte entsprechend folgender der Merkmale (1) Lage und Besonderheiten, (2) Wirtschaftsstruktur, (3) Fachkräftebedarf, (4) Wissenschaft und Forschung, (5) Innovationsstärke sowie (6) Leben und Wohnen. Diesen Merkmalen können insgesamt 25 Indikatoren zugeordnet werden. Je nach Kommune konnten unterschiedlich differenzierte Belege ermittelt werden. Um die Ergebnisse in geeigneter Form präsentieren zu können, wurden sie in der Form von Gemeindesteckbriefen visualisiert (vgl. Gemeindesteckbriefe der Flughafenumfeldregion im Anhang). Als Quellen für die Analyse dienten die Webseiten der Kommunen, Daten des Amtes für Statistik Berlin-Brandenburg und der Arbeitsagentur, Webportale wie Immobilienscout24.com oder Wohnungsboerse.net sowie der Förderkatalog der Bundesregierung. Nach Abschluss der Recherchen wurden die Gemeindesteckbriefe den Kommunen zur abschließenden Prüfung zur Verfügung gestellt und auf deren Hinweise teilweise präzisiert. Eine Ausnahme bildete der Steckbrief für die Gemeinde Grünheide, die aufgrund der Tesla-Ansiedlungsfortschritte später in die Analyse aufgenommen wurde.

\section{Identifikation des Wissenschafts- und Technologiepotenzials}

Am Beginn dieses Arbeitsschrittes stand die Ermittlung von Wissenschaftseinrichtungen mit deren Kompetenzen, gefolgt von der Identifizierung innovativer, technologieorientierter Unternehmen. Leitend für Letztere war eine Definition innovativer, technologieorientierter Unternehmen. Kriterien für die Auswahl der Unternehmen waren entsprechend dieser Definition die Inanspruchnahme von Forschungs- und Entwicklungsfördermaßnahmen auf Ebene des Bundes und/oder des Landes, Innovationskooperationen sowie die Nominierung bzw. das Gewinnen von Innovationspreisen. Für die Identifizierung der Unternehmen wurden verschiedene Recherchewege gewählt:

- Förderkatalog der Bundesregierung (Projekte ab 2010) 
- Übersicht zu Förderungen im Land Brandenburg mit Mitteln des ESF (Brandenburger Innovationsfachkräfte (BIF), Innovationsassistenten) (2014-2020) bzw. des EFRE, Interventionskategorie Forschungs- und Innovationsprozesse in KMU sowie Fortgeschrittene Unterstützungsdienste für KMU und KMU-Zusammenschlüsse (2014-2020)

- Übersicht zu Innovationspreisen Berlin-Brandenburg ab 2010

- Branchenverzeichnisse der Gemeinden

- Webseiten der Technologiezentren in Wildau und Teltow

- Webseiten von Wirtschaftsförderungsgesellschaften, Gemeinden, Zeitungen

- Startup Detector

Die identifizierten Unternehmen, deren Webseiten auf Aktualität geprüft wurden, wurden den Clustern der gemeinsamen Innovationsstrategie der Länder Berlin und Brandenburg sowie den Kommunen der Flughafenumfeldregion zugeordnet. Die Zuordnung zu den Kommunen und Clustern wurde visualisiert, um die Ergebnisse in geeigneter Form kommunizieren zu können. Es erfolgte auch eine Zuordnung der Cluster zu den Kompetenzen der in der Region ansässigen Wissenschaftseinrichtungen, was den Brandenburger Teil der Flughafenumfeldregion betrifft.

Das Wissenschafts- und Technologiepotenzial im Berliner Teil der Flughafenumfeldregion, insbesondere an den Standorten Adlershof und Oberschöneweide, wurde lediglich im Überblick betrachtet und dargestellt, basierend auf einer Auswertung der Webseiten des jeweiligen Standortmanagements.

\section{Ermittlung der Perspektive ausgewählter Akteur/innen und Institutionen}

Um den Status quo der Region aus der Perspektive ausgewählter Regionalakteur/innen kennenzulernen, wurden Expert/innen aus den Bereichen Wirtschaftspolitik, Wirtschaftsförderung, Wissenschaft und Wirtschaft interviewt. Hierzu wurde ein halbstandardisierter Interviewleitfaden erarbeitet, dessen Fragen sich auf die Einschätzung der räumlichen Dimension der Flughafenumfeldregion, das Zusammenspiel von Akteur/innen, die Entwicklung bei Gewerbeflächen und Ansiedlungen, Innovationsthemen sowie Hemmnisse bezogen. Es wurden, bedingt durch die Corona-Pandemie, online/telefonisch sieben Interviews mit zwölf Personen geführt, aufgezeichnet, transkribiert und entlang einem Schema ausgewertet.

\section{Ermittlung von Good Practices in Flughafenumfeldregionen}

Wie bereits weiter oben angedeutet, fokussierte die Ermittlung von Good Practices in Flughafenumfeldregionen auf die Auswertung der Ergebnisse der Arbeiten der Sustainable Airport Areas Initiative 
aus dem Jahr 2018. Über einen Zeitraum von mehreren Jahren hinweg beteiligten sich 70 Experten weltweit in drei Workshops an der Diskussion von Erfahrungen in Flughafenumfeldregionen. In den entsprechenden Guidelines werden Erfahrungen aus den Bereichen Governance, Vision \& Strategie, Regionalplanung und -entwicklung, Mobilität und Erreichbarkeit, Umwelt / Lärm, Wirtschaftliche Entwicklung, Fachkräfteentwicklung und Marketing dargestellt (vgl. IAU, 2018). Den Guidelines wurden Beispiele entnommen, die für die Entwicklung der Flughafenumfeldregion BER aus der Sicht des Bearbeitungsteams von besonderem Interesse sind.

Handlungsansätze und Thesen und digitales Forum

Die Ergebnisse der einzelnen Analyseschritte wurden zusammengefasst und erste Handlungsansätze erarbeitet. Die Synthese wurde in einer Keynote aufbereitet und auf dem Dialogforum am 03. Dezember 2020 vorgestellt. Sie bildete auch die Grundlage für die Erarbeitung von Thesen zur Entwicklung der Flughafenumfeldregion, über die auf dem digitalen Forum von den Teilnehmer/innen abgestimmt wurde.

\section{Ergebnisse}

Nachfolgend werden die Ergebnisse der einzelnen Arbeitsschritte dargestellt.

\subsection{Festlegung der Gebietskulisse der FUR}

Die Region des Flughafenumfeldes wird im Rahmen der vorliegenden Analyse im engeren und im erweiterten Sinne verstanden. Aus dem von beiden Ländern Berlin und Brandenburg erarbeiteten Gemeinsamen Strukturkonzept aus dem Jahr 2007 (vgl. Gemeinsame Landesplanungsabteilung Berlin Brandenburg, 2007) lässt sich die Region im engeren Sinne ableiten. Hierbei umfasst die Region folgende Brandenburger Städte und Gemeinden (vgl. auch Darstellung 2).

- Schönefeld

- Wildau

- Königs Wusterhausen

- Eichwalde

- Schulzendorf

- Zeuthen

- Mittenwalde 
- Blankenfelde-Mahlow

- Rangsdorf

- Großbeeren

- Ludwigsfelde

- Gosen-Neu Zittau

- Flächen in der Gemeinde Teltow

zudem in Berlin folgende Bezirke im Ganzen oder in Teilen:

- Neukölln

- Treptow-Köpenick

- Tempelhof-Schöneberg

- Rummelsburg Blockdammweg (Lichtenberg)

- Spreeufer und Revaler Viereck (Friedrichshain-Kreuzberg)
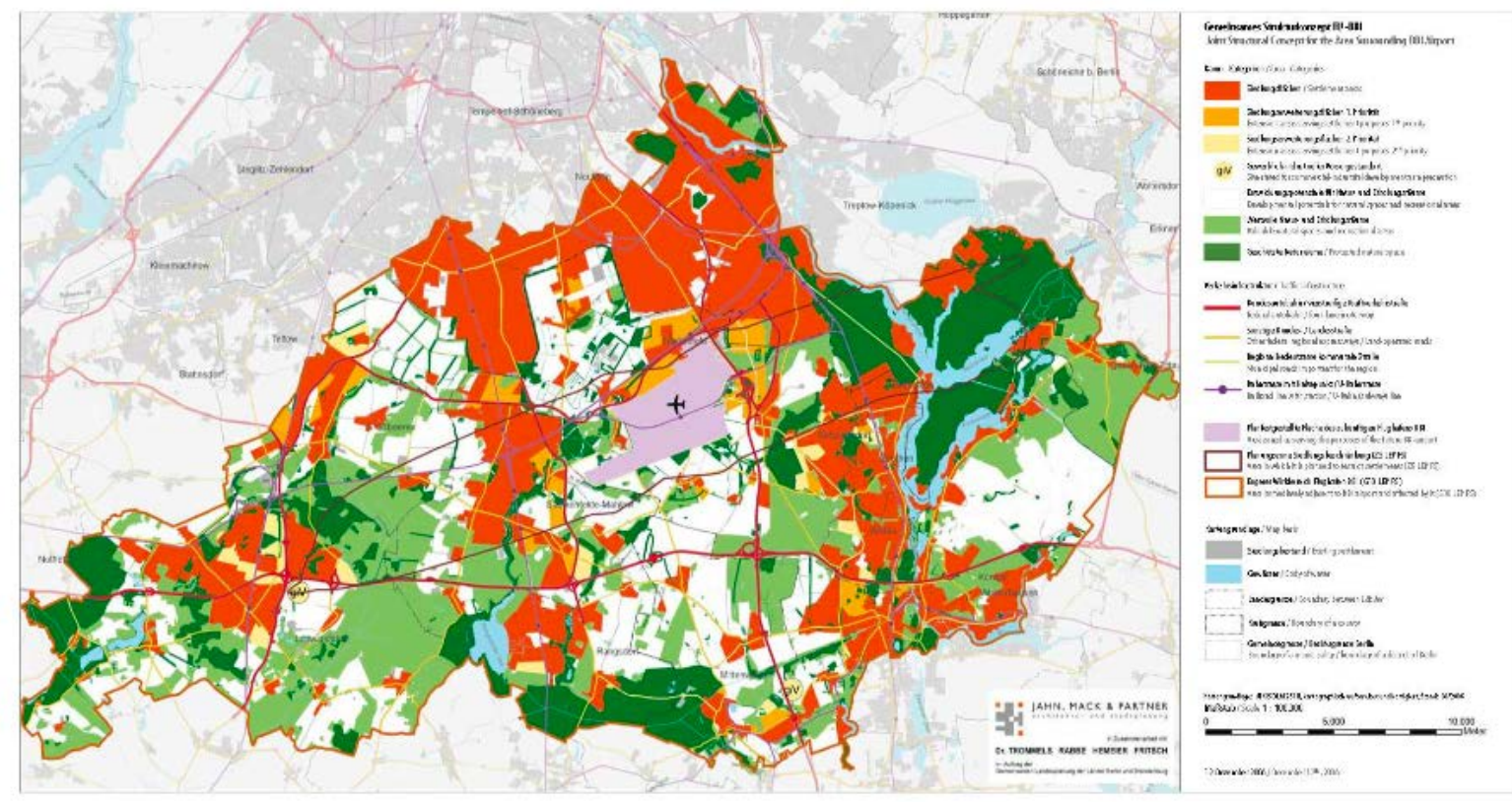

Darstellung 2: Flughafenumfeldregion im Gemeinsamen Strukturkonzept aus dem Jahr 2007 (Quelle: GSK, 2007:13)

In Anbetracht diverser Entwicklungstendenzen in den letzten Jahren und unter Berücksichtigung des Fokus "Wissenschafts- und Innovationspotenzial in der FUR“ wurde im Rahmen der vorliegenden Analyse eine Erweiterung der Flughafenumfeldregion (vgl. Darstellung 3) um Städte und Gemeinden vorgenommen. Hierbei handelt es sich zunächst um zwei Regionale Wachstumskerne. Der Regionale Wachstumskern Fürstenwalde verzeichnete in den vergangenen Jahren ein starkes Wachstum im produzierenden Gewerbe in den Clustern „Verkehr, Mobilität, Logistik“, „Energietechnik“, „Kunststoffe/Chemie“ und „Metall“. Auch die Cluster „Tourismus" und „Gesundheit" haben sich hier sehr 
positiv entwickelt (vgl. Fürstenwalde-Spree, o. J.). Ende 2020 eröffneten die Europa-Universität Viadrina und die TH Wildau ihre gemeinsame Präsenzstelle in Fürstenwalde und stärken damit den Zugang zum Wissenschaftspotenzial sowie den Wissens- und Technologietransfer in dieser Teilregion. Der Regionale Wachstumskern Luckenwalde, der hervorragend verkehrlich an Berlin angebunden ist, wird ebenfalls als zur Flughafenumfeldregion zugehörig betrachtet. Mit dem Ausbau der B 101 beträgt die Fahrzeit BER-Luckenwalde im Motorisierten Individualverkehr etwa 45 Minuten und im Schienenverkehr Berlin-Luckenwalde etwa 30 Minuten. Er kann auf eine steigende Zahl von Arbeitsplätzen verweisen und auch das Pendlersaldo ist nach wie vor positiv. Seine Wirtschaftsstruktur ist geprägt durch wettbewerbsfähige Branchen, insbesondere Gesundheit, Life Sciences, Biotechnologie, Automotive und Metall (vgl. Gruschwitz, 2018). Der RWK ist Standort des Biotechnologieparks und verfügt über eine Präsenzstelle, die gemeinsam von der TH Wildau und der FH Potsdam betrieben wird.

Weiterhin wurden der Flughafenumfeldregion Städte und Gemeinden mit starkem Einwohnerzuwachs zugeordnet. Das betrifft im Landkreis Teltow-Fläming Zossen und Bestensee (vgl. Fischer, 2019). Als Stadt mit sehr guten Bedingungen für qualitativ hochwertiges Wohnen und Arbeiten im Grünen wurde die Kreisstadt Lübben aufgenommen, deren verkehrliche Anbindung nach Berlin ebenfalls als sehr gut bewertet wird und die an einer Entwicklungsachse der Flughafenumfeldregion gelegen ist. Schließlich wurde auch noch die Gemeinde Grünheide als Standort der Gigafabrik von Tesla einbezogen.

In Anbetracht des Untersuchungskontextes, insbesondere aber aufgrund der spezifischen Entwicklungsbedingungen in den Berliner (Teil-) Bezirken wurden diese zwar berücksichtigt und zur Flughafenumfeldregion gezählt, nachfolgende Analysen erfolgten allerdings schwerpunktmäßig zu den Brandenburger Städten und Gemeinden. Gleichwohl findet sich in der Analyse ein Überblick zu den Wissenschafts- und Technologiepotenzialen an den Berliner Standorten Adlershof und Oberschöneweide. 


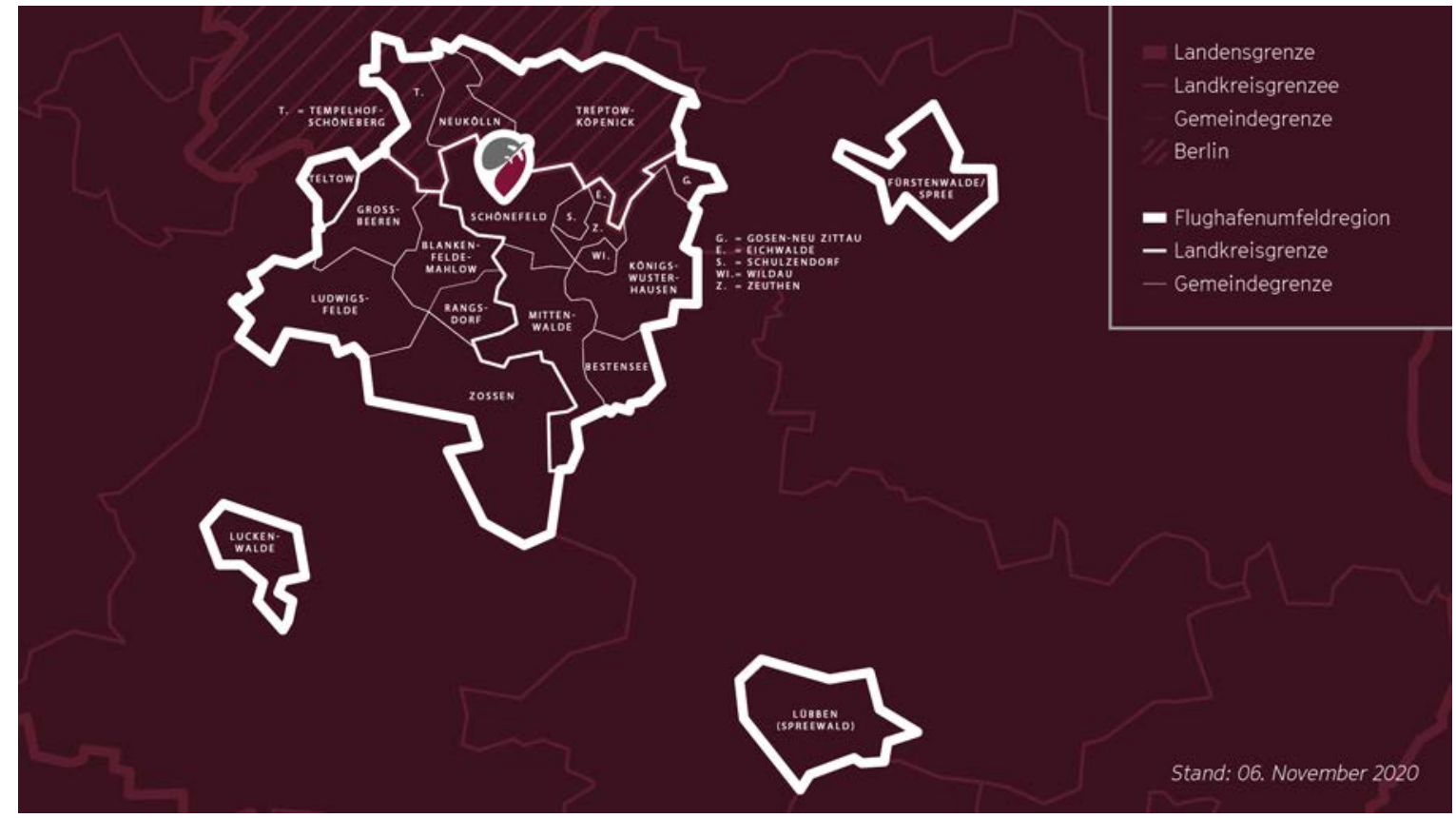

Darstellung 3: Flughafenumfeldregion im erweiterten Verständnis

Für die Erweiterung des Verständnisses der räumlichen Dimension spricht auch die Tatsache, dass sich die Region entlang von Achsen entwickelt, die Autobahnen und größeren Bundesstraßen folgen. Legt man eine 45 Minuten Isochrone zugrunde, befinden sich die weiter oben genannten Städte und Gemeinden ebenfalls in der Flughafenumfeldregion (vgl. Darstellung 4).

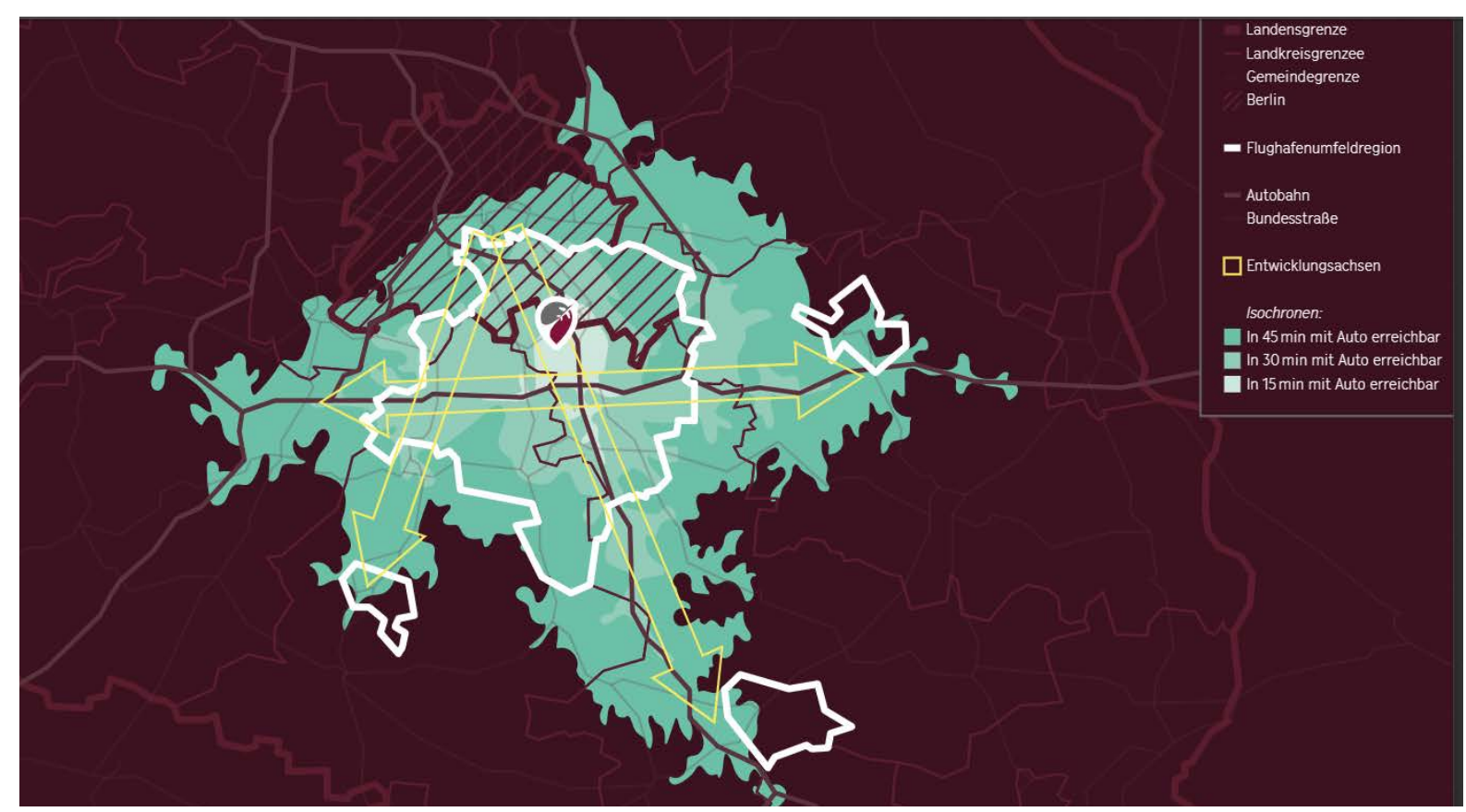

Darstellung 4: Flughafenumfeldregion im erweiterten Verständnis mit Isochrone und Entwicklungsachsen 


\subsection{Charakterisierung der Städte und Gemeinden}

Insgesamt wurden 19 Städte bzw. Gemeinden analysiert und Gemeindesteckbriefe erarbeitet. Die folgende Darstellung 5 zeigt die analysierten Merkmale und zugehörigen Indikatoren.

\begin{tabular}{|c|c|c|c|c|c|c|}
\hline $\begin{array}{l}\text { Lage und } \\
\text { Besonderheiten }\end{array}$ & Wirtschaftsstruktur & Fachkräftebedarf & $\begin{array}{l}\text { Wissenschaft und } \\
\text { Forschung }\end{array}$ & Innovationsstärke & $\begin{array}{l}\text { Leben und } \\
\text { Wohnen }\end{array}$ & $\begin{array}{l}\text { USP - Das } \\
\text { zeichnet die } \\
\text { Gemeinde aus }\end{array}$ \\
\hline $\begin{array}{l}\text { Einwohner/innen } \\
\text { - Bevölkerungs- } \\
\text { dichte } \\
\text { - } \text { Fläche } \\
\text { - Infrastruktur }\end{array}$ & $\begin{array}{l}\text { - Anzahl } \\
\text { Unternehmen } \\
\text { - Ansiedlungen in } \\
\text { den letzten } 3 \\
\text { Jahren } \\
\text { - Branchenschwer- } \\
\text { punkte } \\
\text { - Unternehmens- } \\
\text { größen } \\
\text { - Gewerbeflächen } \\
\text { - Breitbandausbau }\end{array}$ & $\begin{array}{l}\text { - Anzahl offener } \\
\text { Stellen insgesamt } \\
\text { - Branchen mit } \\
\text { überdurchschnitt- } \\
\text { lich hoher } \\
\text { Nachfrage } \\
\text { - Nachfrage nach } \\
\text { hochqualifiziertem } \\
\text { Personal }\end{array}$ & $\begin{array}{l}\text { - Anzahl } \\
\text { Forschungs- } \\
\text { einrichtungen } \\
\text { - Art der } \\
\text { Einrichtungen } \\
\text { - Inhaltliche } \\
\text { Schwerpunkte }\end{array}$ & $\begin{array}{l}\text { - Testbeds } \\
\text { - Spezifische } \\
\text { Netzwerke } \\
\text { - Öffentlich } \\
\text { geförderte } \\
\text { Projekte }\end{array}$ & $\begin{array}{l}\text { - Mietpreisent- } \\
\text { wicklung } \\
\text { - Verfügbarkeit } \\
\text { von Wohnraum } \\
\text { - Medizinische } \\
\text { Versorgung } \\
\text { - Bildungsangebot } \\
\text { - Umweltqualität } \\
\text { - Kulturangebot }\end{array}$ & \\
\hline
\end{tabular}

Darstellung 5: Merkmale und Indikatoren für die Charakterisierung der Städte und Gemeinden

Das Beispiel für einen visualisierten Gemeindesteckbrief zeigt die Darstellung 6. Alle weiteren Gemeindesteckbriefe sind in der Anlage zusammengestellt. 


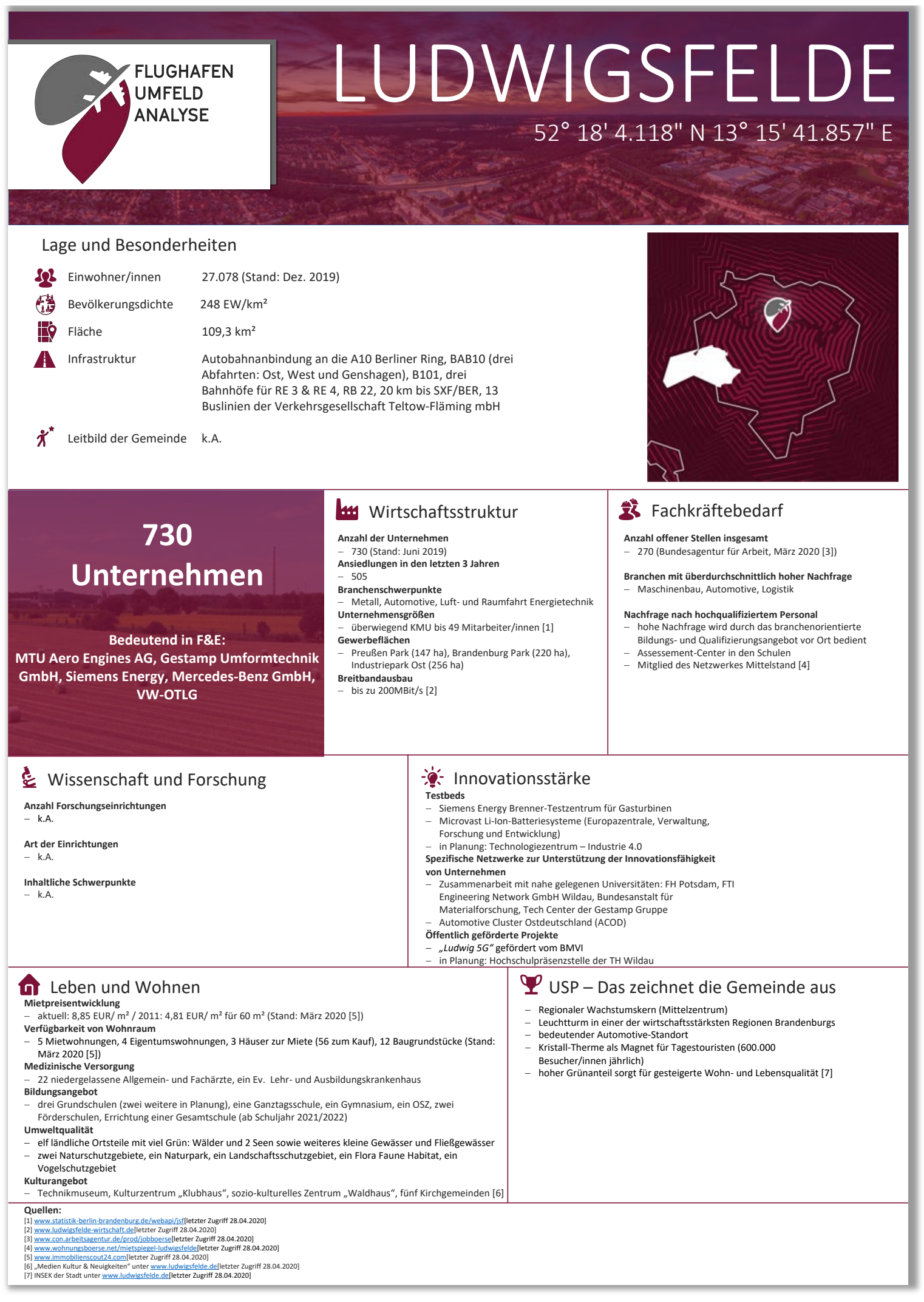

Darstellung 6: Beispiel für einen Gemeindesteckbrief 
Was die Verteilung der Bevölkerung in der Flughafenumfeldregion betrifft, gibt es sieben Städte/Gemeinden mit über 20.000 Einwohner/innen, fünf Städte/Gemeinden mit zwischen 10.000 und 20.000 Einwohner/innen sowie sieben Städte/Gemeinden mit unter 10.000 Einwohner/innen. Die Flughafenumfeldregion hatte mit Stand März 2020 insgesamt 308.802 Einwohner/innen (vgl. Darstellung 7).

\begin{tabular}{|c|c|c|c|c|c|}
\hline \multicolumn{4}{|c|}{ Anzahl Einwohner/innen } & & \\
\hline $\begin{array}{l}\text { Sieben Städte/Gemeir } \\
\text { Einwohner/innen } \\
\text { Königs Wusterhausen } \\
\text { Fürstenwalde } \\
\text { Blankenfelde-Mahlow } \\
\text { Ludwigsfelde } \\
\text { Teltow } \\
\text { Luckenwalde } \\
\text { Zossen }\end{array}$ & $\begin{array}{l}\text { len mit mehr als } 20.000 \\
37.812 \text { (RWK*) } \\
32.000 \text { (RWK) } \\
28.087 \\
26.833 \text { (RWK) } \\
26.963 \\
20.567 \text { (RWK, Kreisstadt) } \\
19.891\end{array}$ & 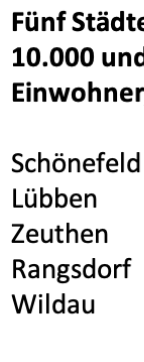 & $\begin{array}{l}\text { /Gemeinden zwischen } \\
20.000 \\
\text { /innen } \\
16.434\left(\mathrm{RWK}^{*}\right) \\
14.044 \\
11.419 \\
11.360 \\
10.429\left(\mathrm{RWK}^{*}\right)\end{array}$ & $\begin{array}{l}\text { Sieben Städte/Gen } \\
\text { unter } \mathbf{1 0 . 0 0 0 ~ E i n w} \\
\text { Mittenwalde } \\
\text { Schulzendorf } \\
\text { Großbeeren } \\
\text { Bestensee } \\
\text { Eichwalde } \\
\text { Gosen-Neu Zittau } \\
\text { Grünheide (Mark) }\end{array}$ & $\begin{array}{l}\text { en } \\
\text { r/innen } \\
9.278 \\
8.536 \\
8.607 \\
8.046 \\
6.423 \\
3.257 \\
8.800^{1}\end{array}$ \\
\hline Insgesamt: 308.786 & & & & & $\begin{array}{l}\text { März } 2020 \\
\text { Dez. } 2019\end{array}$ \\
\hline
\end{tabular}

Darstellung 7: Bevölkerung in der Flughafenumfeldregion

Im Hinblick auf den Unternehmensbesatz in den Städten und Gemeinden der Flughafenumfeldregion und die Ansiedlung/Gründung von Unternehmen in den letzten drei Jahren, fällt auf, dass es sieben Städte/Gemeinden mit über 1.000 Unternehmen gibt, angeführt von der Stadt Königs Wusterhausen mit 3.046 Unternehmen (vgl. Darstellung 8).

\begin{tabular}{|c|c|c|c|c|c|}
\hline \multicolumn{4}{|c|}{ Unternehmensbesatz/Ansiedlungen in den letzten drei Jahren } & & \\
\hline \multicolumn{4}{|c|}{ Städte/Gemeinden mit mehr als 1000 Unternehmen } & \multicolumn{2}{|c|}{$\begin{array}{l}\text { Städte/Gemeinden mit stärkstem Zuwachs in den letzte drei Jahren } \\
\text { (über 50\%) }\end{array}$} \\
\hline Königs Wusterhausen & 3.046 & Rangsdorf & 449 & & \\
\hline Schönefeld & 2.475 & Schulzendorf & 445 & Zossen & 116,0 (Werte nicht plausibel) \\
\hline Fürstenwalde & 2.271 & Mittenwalde & 445 & Ludwigsfelde & 72,7 \\
\hline Luckenwalde & 1.874 & Bestensee & 303 & Teltow & 71,4 \\
\hline Teltow & 1.293 & Eichwalde & 293 & Gosen-Neu Zittau & 64,6 \\
\hline Blankenfelde-Mahlow & 1.171 & Gosen-Neu Zittau & 212 & Bestensee & 63,8 \\
\hline Zossen & 1.149 & & & Blankenfelde-Mahlow & 62,9 \\
\hline Ludwigsfelde & 730 & & & Rangsdorf & 60,1 \\
\hline Lübben & 693 & & & Mittenwalde & 55,6 \\
\hline Wildau & 678 & & & Wildau & 52,5 \\
\hline Zeuthen & 509 & & & & \\
\hline Großbeeren & 504 & & & & \\
\hline \multicolumn{6}{|l|}{ Insgesamt: 19.253} \\
\hline \multicolumn{4}{|c|}{$\begin{array}{l}9 \text { Städte/Gemeinden mit überwiegend sehr kleinen Unternehmen bis max. } 9 \\
\text { Beschäftigten, z. B. Bestensee } \\
9 \text { Städte/Gemeinden mit überwiegend kleinen Unternehmen bis max. } 49 \\
\text { Beschäftigten, z. B. Schönefeld } \\
\text { Einige wenige Unternehmen mit mehr als } 250 \text { Beschäftigten, z. B. } \\
\text { Ludwigsfelde }\end{array}$} & \multicolumn{2}{|c|}{$\begin{array}{l}\text { Die Unternehmen sind zahlreichen unterschiedlichen Branchen } \\
\text { zugeordnet. }\end{array}$} \\
\hline
\end{tabular}

Darstellung 8: Unternehmensbesatz und Unternehmensansiedlung in der Flughafenumfeldregion (statistik Berlin Brandenburg, 2020) 
Was den Zuwachs und damit die Dynamik des Ansiedlungsgeschehens angeht, sind Ludwigsfelde und Teltow mit 72,7 bzw. 71,4 Prozent führend (vgl. Darstellung 8). Es handelt sich überwiegend um kleine Unternehmen, insbesondere in Ludwigsfelde und Blankenfelde-Mahlow sind auch einige größere Unternehmen ansässig. Grünheide mit der geplanten Gigafactory von Tesla wird der mit Abstand größte Unternehmensstandort in der Flughafenumfeldregion werden. Tesla beabsichtigt, bis zu 12000 Menschen in seiner Gigafactory zu beschäftigen (vgl. Meyer, 2020), was zu einem sehr großen Fachkräftebedarf in der Region führen wird.

Dieser stellte sich, unabhängig von der Entwicklung bei Tesla, im Februar 2020 folgendermaßen dar (vgl. Darstellung 9). Insgesamt waren zum 27.02.2020 3.078 offene Stellen in der Region vorhanden. Städte und Gemeinden mit über 200 offenen Stellen waren Teltow, Großbeeren, Königs Wusterhausen, Schönefeld, Ludwigsfelde, Wildau und Luckenwalde (vgl. Darstellung 9). Branchen mit hoher Nachfrage waren Logistik, Gesundheit- und Pflege, Automotive und Maschinenbau sowie das Handwerk. Die Nachfrage nach hochqualifizierten Personen richtete sich insbesondere auf Ingenieur/innen und Pädagog/innen und Erzieher/innen. Sie wird sich mit der Inbetriebnahme der Teslafabrik weiter verstärken und aus der Region nicht befriedigt werden können. Im Ergebnis entsteht in der Flughafenumfeldregion ein starker Wettbewerb um Ingenieur/innen, aber auch andere Fachkräfte.

\begin{tabular}{|c|c|}
\hline Fachkräftebedarf & \\
\hline 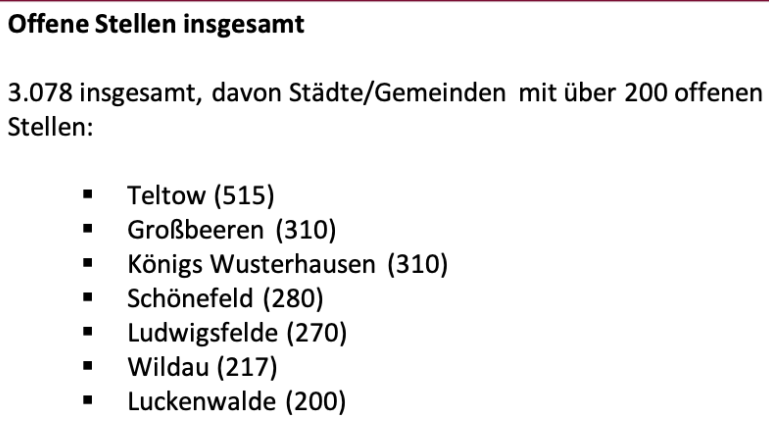 & $\begin{array}{l}\text { Branchen mit hoher Nachfrage: } \\
\text { - Logistik } \\
\text { - Gesundheits- und Pflegbranche } \\
\text { - Hatomotive und Maschinenbau } \\
\text { Nachfrage nach hochqualifizierten Personen } \\
\text { (vergleichsweise an wenigen Standorten), insbesondere } \\
\text { - Ingenieur/innen } \\
\text { - Pädagog/innen und Erzieher/innen }\end{array}$ \\
\hline
\end{tabular}

Darstellung 9: Fachkräftebedarf in ausgewählten Städten/Gemeinden der Flughafenumfeldregion (Arbeitsagentur, 2020)

Dies wird auch dazu führen, dass sich die Situation im Bereich Wohnen in der Region weiter verschärfen wird. Mit Stand März 2020 waren in der Flughafenumfeldregion in den 19 Städten/Gemeinden lediglich 234 Mietwohnungen und 869 Häuser im Angebot (vgl. Darstellung 10). Auch die Mietpreissituation deutet auf eine problematische Lage hin. Mietpreise über 10 Euro pro Quadratmeter sind keine 
Ausnahme mehr und in den meisten Kommunen der Flughafenumfeldregion liegen die Mietpreise bei über 6,52 bis zu 10,00 Euro pro Quadratmeter.

\begin{tabular}{|l|l|}
\hline Wohnangebote (Anzahl Mietwohnungen, Häuser zum Kauf) & Mietpreise \\
\hline Mietwohnungen insgesamt im Angebot: & Mietpreise über 10 Euro/m2 \\
228 davon & Zeuthen \\
41 in Fürstenwalde, & Wildau \\
36 in Schönefeld und & Teltow \\
24 in Wildau & Schönefeld \\
& Rangsdorf \\
& Blankenfelde-Mahlow \\
\hline Häuser insgesamt im Angebot: & Grünheide \\
869 davon & Mietpreise 6,52 bis unter 10 Euro/m2 \\
& Schulzendorf \\
180 in Königs Wusterhausen und & Großbeeren \\
97 in Blankenfelde-Mahlow & Mittenwalde \\
& Zossen \\
& Bestensee \\
& Gosen-Neu Zittau \\
& Fürstenwalde \\
& Luckenwalde \\
& Lübben \\
& \\
\hline
\end{tabular}

Darstellung 10: Wohnungsangebote und Mietpreise (Daten entnommen aus: Immobilienscout24, wohnungsboerse.net, 2020)

Zum Vergleich: Der durchschnittliche Mietpreis für eine $60 \mathrm{~m}^{2}$ Wohnung lag im Jahr 2020 in Berlin bei 14,20 Euro, im Land Brandenburg bei 7,21 Euro und in Deutschland bei 8,27 Euro (vgl. Wohnungsbörse, 2020).

Die Auswertung der Gemeindesteckbriefe lässt erkennen, dass die Daseinsvorsorge in der Region gut entwickelt ist. Sowohl Bildungs- als auch Kulturangebote und die medizinische Versorgung werden als ausreichend eingeschätzt. Durch die Nähe zur Metropole Berlin erhöht sich die Attraktivität des Brandenburger Teils der Flughafenumfeldregion bezüglich der Daseinsvorsorge noch zusätzlich. In den Kreisstädten Lübben und Luckenwalde sowie im Bereich der RWKs Königs Wusterhausen, Wildau, Schönefeld, Fürstenwalde, Ludwigsfelde und Luckenwalde sind gute Angebote der Daseinsvorsorge gegeben sowie attraktive Kulturangebote vorhanden. In Luckenwalde, Lübben, Ludwigsfelder, Königs Wusterhausen und Fürstenwalde gibt es Krankenhäuser und in Waltersdorf, Wildau und auch Rangsdorf große und mittlere Einkaufszentren.

In den Gemeindesteckbriefen sind auch beispielhafte Aussagen zu besonderen Innovationsstärken der Region getroffen worden (vgl. Darstellung 11). An dieser Stelle sei auf die gut mit technologieorientierten Unternehmen ausgelasteten Technologiezentren in Wildau, Teltow und Luckenwalde verwiesen. Hervorgehoben werden können weiterhin das Siemens Energy Brenner-Testzentrum für Gasturbinen und die neue Europazentrale von Microvast Li-Ion-Batteriesysteme in Ludwigsfelde sowie das XLTestbed von Rolls Royce „Big Data“ - Engine Health Monitoring bei Geschäftsflugzeugen in Blankenfelde-Mahlow. 
- mehrere gut mit technologieorientierten Unternehmen ausgelastete Technologiezentren und Netzwerk (Wildau, Teltow und Luckenwalde)

- Turbinen-Teststände bei Anecom (Wildau)

- Siemens Energy Brenner-Testzentrum für Gasturbinen (Ludwigsfelde)

- Microvast Li-lon-Batteriesysteme, Europazentrale (Verwaltung, FuE) (Ludwigsfelde)

- Automotive Cluster Ostdeutschland (ACOD) (Ludwigsfelde)

— Projekt: „Ludwig 5G“ gefördert vom BMVI (Ludwigsfelde)

_ XL-Testbed von Rolls Royce: "Big Data" - Engine Health Monitoring bei Geschäftsflugzeugen (Blankenfelde-Mahlow)

- Biotechnologiepark (Luckenwalde)

- Hochschulpräsenzstelle der TH Wildau und FH Potsdam mit Coworkingspace, Showroom und Makerspace (Luckenwalde)

Darstellung 11: Ausgewäh/te Innovationsstärken (vgl. Aussagen zu Innovationsstärken in Gemeindesteckbriefen im Anhang)

Betrachtet man die für die einzelnen Kommunen erhobenen Alleinstellungsmerkmale ergeben sich folgende „Typen“ von Städten/Gemeinden:

- Städte und Gemeinden als naturnahe Erholungs- und Tourismusstandorte mit hohem Anteil an Gewässern (z. B. Gosen-Neu Zittau, Zossen, Bestensee, Fürstenwalde)

- Standorte mit attraktiver Wohnlage (z. B. Eichwalde, Zeuthen, Lübben)

- wirtschaftlich starke Standorte mit Sitz von großen Unternehmen und gut ausgelasteten Gewerbegebieten (z. B. Ludwigsfelde, Blankenfelde-Mahlow)

- Standorte mit etablierten Technologiezentren (z. B. Teltow, Wildau, Luckenwalde)

Hinzu kommen die Gemeinde Schönefeld als Flughafenstandort, die Gemeinde Großbeeren als Standort eines bedeutenden Güterverkehrszentrums, die Gemeinde Grünheide (Mark) als Standort von Tesla und Wildau als Hochschulstadt.

\subsection{Identifikation des Wissenschafts- und Technologiepotenzials}

Mit Wissenschaftseinrichtungen in Adlershof sowie den innovativen, technologieorientierten Unternehmen in Adlershof und Oberschöneweide verfügt der Berliner Teil der Flughafenumfeldregion über ein einzigartiges Wissenschafts- und Technologiepotenzial. Es konzentriert sich im Wissenschafts- und Technologiepark Adlershof in den fünf Technologiezentren (vgl. Darstellung 12) sowie den außeruniversitären und universitären Forschungseinrichtungen (vgl. Darstellung 13). 


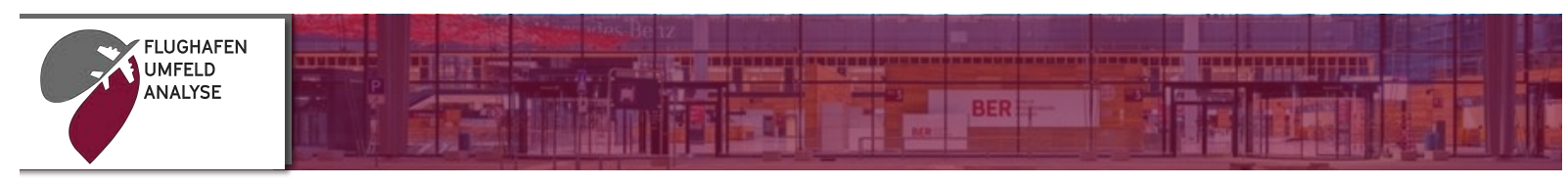

Wissenschafts- und Technologiepark Adlershof | Technologiezentren

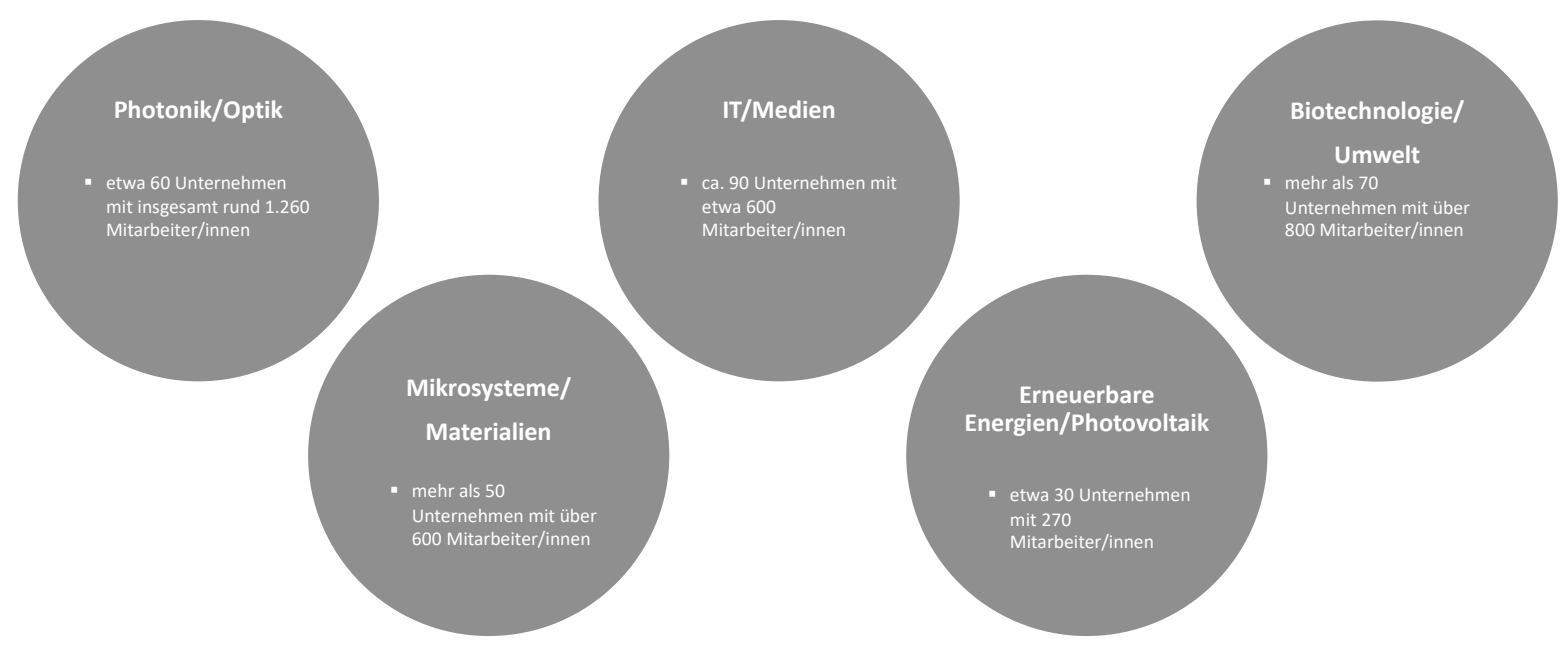

Darstellung 12: Technologiezentren im Berliner Teil der Flughafenumfeldregion (Adlershof a), b), c), d), e), 2020)

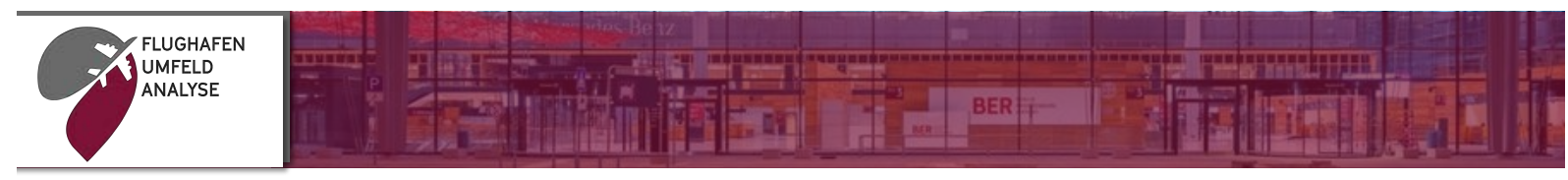

Wissenschafts- und Technologiepark Adlershof | Außeruniversitäre Forschungseinrichtungen und Campus der Humboldt-Universität

\begin{tabular}{|ll|}
\hline - & Bundesanstalt für Materialfforschung und -prüfung (Analytische Chemie, Referenzmaterialien) \\
\hline - & Brandenburgische Technische Universiät Cottbus-Senftenberg, Arbeitsgebiet Luftchemie und Luftreinhaltung \\
\hline - & Deutsches Zentrum für Luft- und Raumfahrt (Planetenforschung, Verkehrssysteme, Optische Sensoren) \\
\hline - & Ferdinand-Braun-Institut, Leibniz-Institut für Höchstfrequenztechnik \\
\hline - & Helmholtz-Zentrum Berlin für Materialien und Energie \\
\hline - & Leibniz-Institut für Kristallzüchtung \\
\hline - & Leibniz-Institut für Analytische Wissenschaften (optischen Spektroskopieverfahren für die Material- und \\
\hline - & Grenzzllächenanalytytik) \\
\hline - & Physikalisch-Irstituchnische Bundesanstalt (Photonenradiometrie) \\
\hline
\end{tabular}

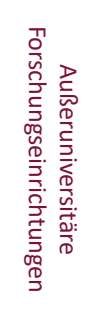

Institute für für Chemie, Geographie, Informatik, Mathematik, Physik und Psychologie mit Sitz in Adlershof
Schwerpunkte:
: Moderne Optik,
Molekulare Systeme,
Mathematische Physik und Wissenschaftliches Rechnen

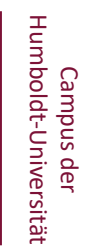

Darstellung 13: Forschungseinrichtungen im Berliner Teil der Flughafenumfeldregion (Adlershoff) 2020)

Hinzu kommen die beiden Technologiestandorte in Oberschöneweide (vgl. Darstellung 14). 


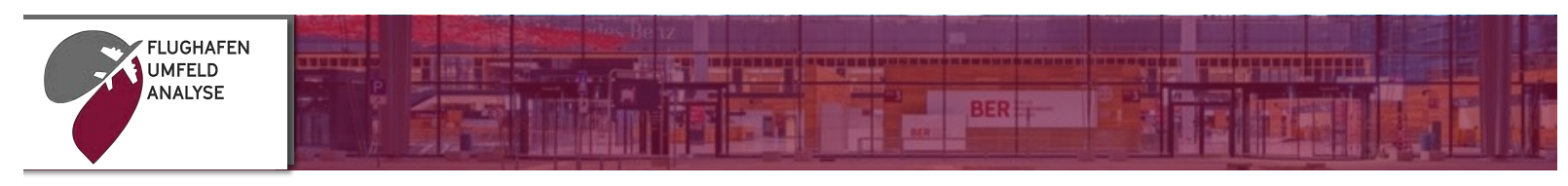

Weitere Technologiestandorte im Berliner Teil der FUR: Oberschöneweide

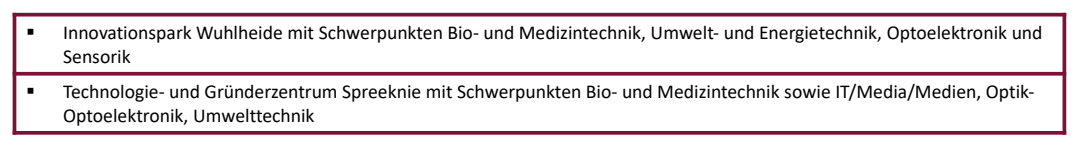

Darstellung 14: Technologiestandorte in Berliner Teil der Flughafenumfeldregion (Oberschöneweide) (Innovationspark Wuhlheide, 2020, Technologie- und Gründerzentrum Spreeknie, 2020)

Im Unterschied zum Berliner Teil der Flughafenumfeldregion gab es bislang für den Brandenburg Teil keine kompakte Übersicht zu den Wissenschafts- und Technologiepotenzialen, die hier auch räumlich weniger konzentriert sind als im Berliner Teil. Die Darstellung 15 zeigt die Forschungseinrichtungen und Technologiezentren im Brandenburger Teil der Flughafenumfeldregion mit ihren Kompetenzen, der Standort Adlershof ist lediglich angedeutet, da der Fokus der Betrachtung auf dem Brandenburg Teil der Flughafenumfeldregion liegt.

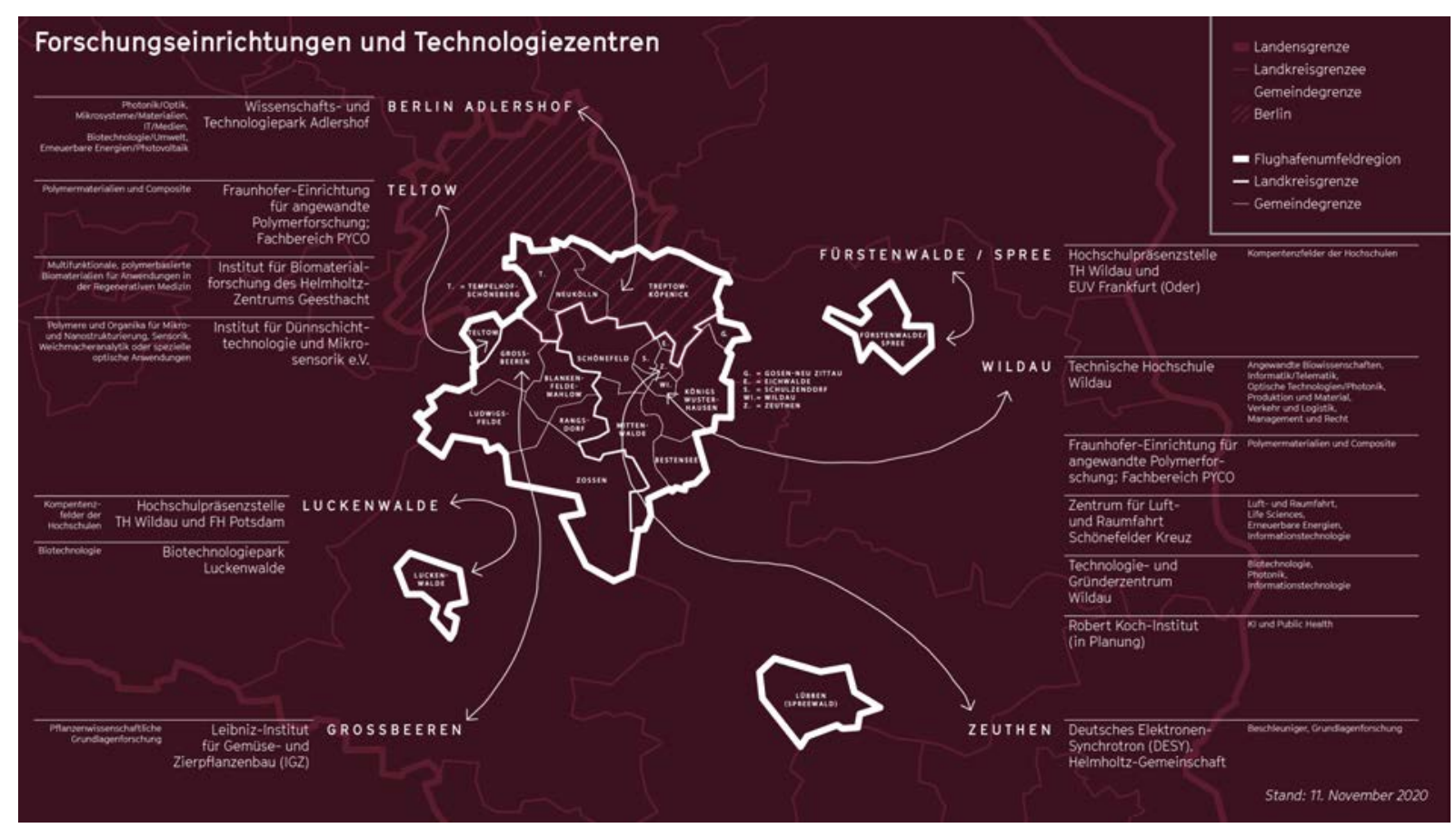

Darstellung 15: Forschungseinrichtungen und Technologiezentren im Brandenburger Teil der Flughafenumfeldregion

Um das Innovationspotenzial des Brandenburger Teils der Flughafenumfeldregion über die ansässigen Wissenschafts- und Forschungseinrichtungen hinaus zu charakterisieren und deren Passfähigkeit zu den Profilen der ansässigen Unternehmen abzuschätzen, wurden innovative, technologieorientierte Unternehmen dieser Teilregion identifiziert. 
Unter Innovationspotenzial in der Region werden hierbei Ressourcen und Kompetenzen verstanden, die in der Anzahl und Ausrichtung der Wissenschaftseinrichtungen sowie der innovativen, technologieorientierten Unternehmen in einem ersten Schritt sichtbar gemacht werden können. Eine tiefergehende Bewertung des Potenzials würde die Bewertung der Ressourcen und Kompetenzen quantitativ und qualitativ sowie vergleichend mit anderen Regionen erfordern.

Unter innovativen, technologieorientierten Unternehmen werden solche Unternehmen verstanden, deren Leistungsangebote auf neuen technologischen Ideen und Forschungsergebnissen basieren und in denen die Entwicklung und Vermarktung neuer Technologien wesentlicher Bestandteil der Unternehmensstrategie ist (vgl. Wagner und Schultz, 2011:30). Sie bilden einen Teil der innovativen Unternehmen / Innovatoren, also derjenigen Unternehmen, die innerhalb eines zurückliegenden Zeitraumes von drei Jahren zumindest eine Produkt- oder Prozessinnovation eingeführt haben (vgl. ZEW, 2019:14), wobei bei dieser Analyse vor allem nach Unternehmen mit Produktinnovationen gesucht wurde. Voraussetzung, um als Unternehmen in diese Auswahl aufgenommen zu werden, ist demzufolge die eigene Durchführung von Forschung und/oder Entwicklung bzw. die Beteiligung daran. Kriterien hierfür waren die Inanspruchnahme von Forschungs- und Entwicklungsfördermaßnahmen auf Ebene des Bundes und des Landes, Innovationskooperationen mit Wissenschaftseinrichtungen sowie die Nominierung bzw. das Gewinnen von Innovationspreisen (siehe methodisches Vorgehen in Kapitel 2).

Nachdem die innovativen, technologieorientierten Unternehmen per Recherchen identifiziert wurden, erfolgte deren Zuordnung zu den Clustern der gemeinsamen Innovationsstrategie der Länder BerlinBrandenburg. Hierzu zählen die Cluster (vgl. WFBB, o. J.):

- Verkehr, Mobilität, Logistik (Automotive, Logistik, Luftfahrttechnik, Schienenverkehrstechnik, Verkehrstelematik)

- Energietechnik

- Ernährungswirtschaft

- Gesundheitswirtschaft (Biotechnologie/Pharma, eHealth/Telemedizin, Kliniken, Medizintechnik, Rehabilitation)

- Kunststoff und Chemie

- Medien, IKT, Kreativwirtschaft (IKT - Telekommunikation, Softwareentwicklung; Digitale Medien, Games; Film, TV; Geoinformationswirtschaft; Sicherheit mit IT, IT-Sicherheit)

- Metall (Metallerzeugung, Metallver- und bearbeitung, Maschinenbau)

- Optik und Photonik

Zusätzlich wurden die Kategorien „Mess- und Automatisierungstechnik, Chips, Sensoren“ sowie „Umwelttechnik" gebildet, um eine umfassendere Zuordnung der Unternehmen zu ermöglichen, da sich 
einige Unternehmen nicht den obigen Clustern zuordnen lassen. Unternehmen aus dem Bereich der Bioverfahrenstechnik wurden dem Cluster Gesundheitswirtschaft zugeordnet. Das Ergebnis der Analyse ist in der Darstellung 16 dokumentiert.

Ermittelt wurde darüber hinaus, ob diese Unternehmen auf dem Gebiet der Patentierung aktiv sind. Hierfür wurde die Online-Patentdatenbank PatBase, entwickelt von Minesoft in Zusammenarbeit mit der RWS Group, genutzt (vgl. PatBase, o. J.). Die Datenbank ermöglicht den Zugang zu mehr als 100 Millionen Publikationen über Patentanmeldungen, Patenterteilungen, Gebrauchsmuster und Designs aus mehr als 100 Patentämtern (vgl. PatBase, o. J.).

Gesucht wurde nach Patentanmeldungen, ein Vorgehen, das bei Analysen zum Innovationspotenzial von Unternehmen und Regionen häufig angewendet wird (vgl. z. B. Bittelmeyer et al., 2007; Harhoff, 2010; Deutsches Patent- und Markenamt, o. J.; Henn und Werner, 2016). Patentanmeldungen deuten darauf hin, dass ein ökonomisches Verwertungsinteresse an einer Erfindung besteht, unabhängig davon, ob die Erfindung zu einem Produkt oder ökonomischen Erfolg geführt wird (vgl. Schibany und Dachs, 2003:5). Sie geben insofern Hinweise auf Innovationsaktivitäten und technologische Entwicklungen.

Die Suche nach Patentanmeldungen der bereits identifizierten innovativen, technologieorientierten Unternehmen erfolgte unter Verwendung des aktuellen Unternehmensnamens. Gesucht wurde damit der/die Patentanmelder/in und von ihm/ihr angemeldete Erfindungen (Anzahl Patenfamilien), wobei die Anzahl von Anmeldungen in vielen Fällen höher als die Zahl der Erfindungen ist, da für diese mehrere Anmeldungen getätigt werden können. Zu beachten ist auch, dass Patentanmeldungen, die unter einem früheren Unternehmensnamen oder von einem verwandten Unternehmen angemeldet wurden, nicht berücksichtigt sind. Für ausgewählte große Unternehmen mit Standorten in der Flughafenumfeldregion wurde recherchiert, ob deren Tochterunternehmen oder Niederlassungen möglicherweise an den Patentanmeldungen beteiligt sind. Prinzipiell ist eine eindeutige Zuordnung der Patentanmeldungen von Unternehmen zu einzelnen Standorten nicht möglich. Der Grund dafür besteht darin, dass Anmeldungssitz und Erfindungsort nicht übereinstimmen müssen (vgl. Henn und Werner, 2016:9). Die Suche erfolgte für den Zeitraum 2010-2019. Letzter Stand der Suche in der Datenbank PatBase ist der 04.09.2020. Im Zeitraum 2010-2019 haben 36 Unternehmen aus der Flughafenumfeldregion mindestens eine Erfindung zum Patent angemeldet. Das entspricht einem Anteil von rund 32 Prozent der innovativen, technologieorientierten Unternehmen der Region (siehe Darstellung 16). Hierbei ist zu berücksichtigen, dass es große Unternehmen mit sehr vielen Patentanmeldungen geben kann, die ihren Sitz außerhalb der Region haben, aber auch an ihrem Standort in der Flughafenumfeldregion Patentaktivitäten entfalten. Das trifft beispielsweise auf die Rolls-Royce Deutschland Ltd \& Co KG zu. In einer Pressemitteilung aus dem Jahr 2017 wird darauf verwiesen, dass das Unternehmen an 
seinem Standort in Dahlewitz jährlich über 60 Patente anmeldet (vgl. Fischer, 2017). Insgesamt werden für das Unternehmen im Zeitraum 2010-2019 in der Datenbank PatBase 849 Patentanmeldungen ausgewiesen. Da ein erheblicher Anteil dieser Patentanmeldungen dem Standort in der Flughafenumfeldregion zugeordnet werden kann, wird das Unternehmen als patentierendes Unternehmen für die Region gezählt. Gleichwohl kann die Anzahl der Patentanmeldungen von Rolls-Royce Deutschland nicht allein dem Standort in Dahlewitz zugeordnet werden.

Nicht gezählt werden hingegen Unternehmen, die zwar über sehr viele Patentanmeldungen verfügen, es aber keine Hinweise auf Patentaktivitäten in der Flughafenumfeldregion gibt, wie z. B. bei der Schaeffler Technologies AG \& Co. KG. Für dieses Unternehmen weist die Datenbank PatBase insgesamt 15.841 Patenanmeldungen im Zeitraum 2010-2019 aus.

Einzelne Unternehmen der Region tauchen in der Patentstatistik nicht auf, vermutlich da sie unter dem Namen der Mutterfirma patentieren. Das trifft in der Region auf die Firma MTU maintanance BerlinBrandenburg zu. Auch für die Mercedes-Benz Ludwigsfelde GmbH konnten mit diesem Vorgehen keine Patentanmeldungen identifiziert werden.

Die folgende Darstellung 16 zeigt die Zuordnung der innovativen, technologieorientierten Unternehmen zu den Clustern der Wirtschaftsförderung Brandenburg (leicht modifiziert) sowie die Anzahl von Unternehmen mit Patentanmeldungen im Zeitraum 2010-2019 im Überblick.

\begin{tabular}{|c|c|c|}
\hline Cluster (in Anlehnung an WFBB) & $\begin{array}{c}\text { Anzahl } \\
\text { Unternehmen }\end{array}$ & $\begin{array}{l}\text { Unternehmen mit } \\
\text { Patentanmeldungen } \\
\text { (2010-2019) }\end{array}$ \\
\hline $\begin{array}{l}\text { Verkehr, Mobilität, Logistik (Automotive, Logistik, Luftfahrttechnik, } \\
\text { Schienenverkehrstechnik, Verkehrstelematik) }\end{array}$ & 12 & 3 \\
\hline Energietechnik & 7 & 1 \\
\hline $\begin{array}{l}\text { Gesundheitswirtschaft (Biotechnologie/Pharma, eHealth/Telemedizin, Kliniken, } \\
\text { Medizintechnik, Rehabilitation) }\end{array}$ & 27 & 14 \\
\hline Kunststoff und Chemie & 11 & 3 \\
\hline $\begin{array}{l}\text { IKT, Medien, Kreativwirtschaft (IKT - Telekommunikation, Softwareentwicklung; } \\
\text { Digitale Medien, Games; Film, TV; Geoinformationswirtschaft; Sicherheit mit IT, IT- } \\
\text { Sicherheit) }\end{array}$ & 17 & 3 \\
\hline Metall (Metallerzeugung, Metallver- und -bearbeitung, Maschinenbau) & 13 & 4 \\
\hline Optik und Photonik & 8 & 2 \\
\hline Mess- und Automatisierungstechnik, Chips, Sensoren & 7 & 3 \\
\hline Umwelttechnik & 3 & 0 \\
\hline Sonstige & 7 & 3 \\
\hline Gesamt & 112 & 36 \\
\hline
\end{tabular}

Darstellung 16: Innovative, technologieorientierte Unternehmen im Brandenburger Teil der Flughafenumfeldregion nach Clustern und Patentaktivitäten (Stand 04.09.2020)

Die Analyse zeigt mit 27 Unternehmen einen Schwerpunkt im Cluster Gesundheitswirtschaft. Auch die Cluster Medien, IKT, Kreativwirtschaft (17), Metall (13), Verkehr, Mobilität Logistik (12) und Kunststoff und Chemie (11) sind mit mehr als 10 Unternehmen vertreten. 
Die Zuordnung der innovativen, technologieorientierten Unternehmen zu den Städten/Gemeinden der Flughafenumfeldregion ergibt folgendes Bild (vgl. Darstellung 17).

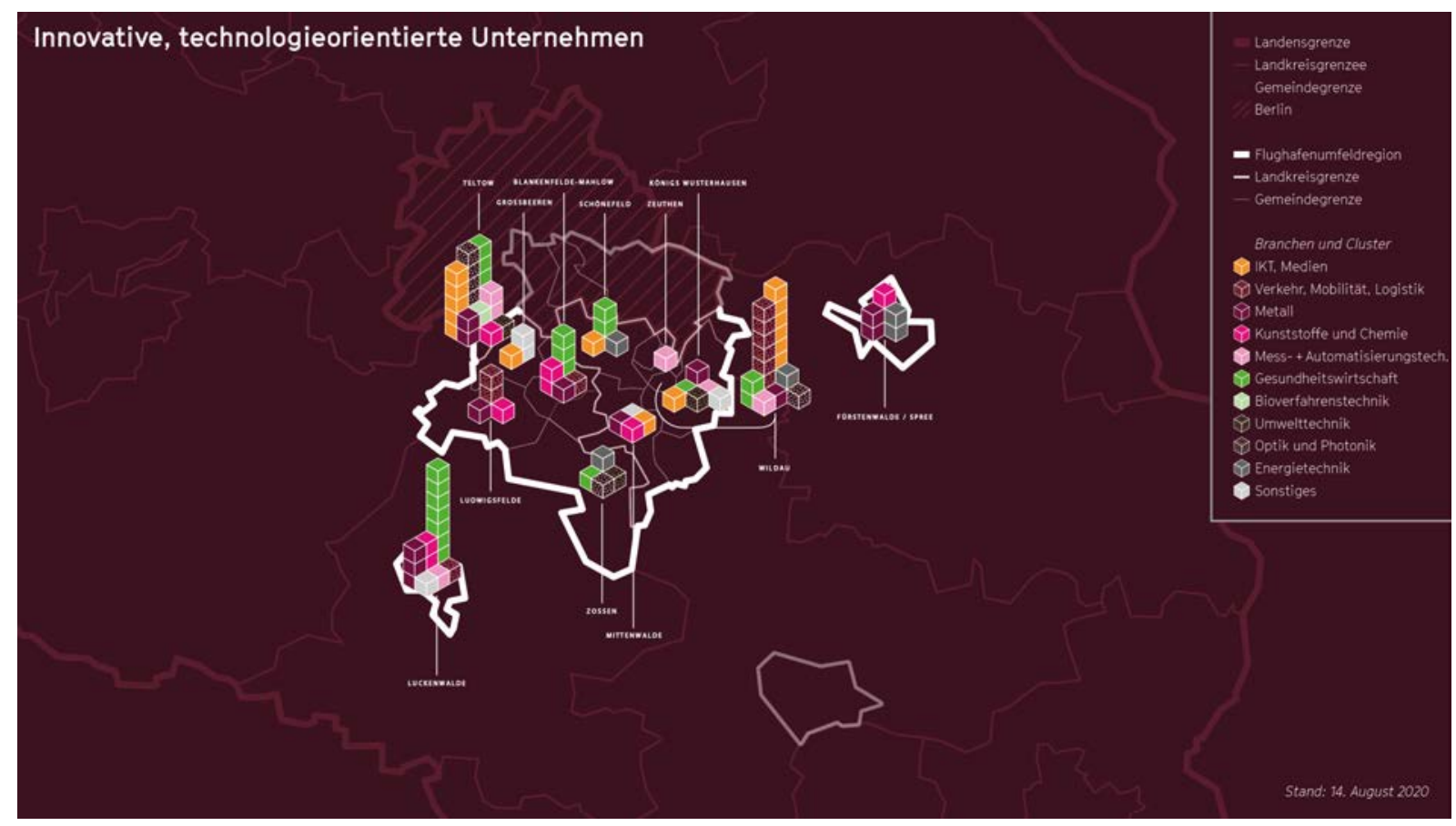

Darstellung 17: Innovative, technologieorientierte Unternehmen nach Clustern sowie Städten/Gemeinden

Die meisten innovativen, technologieorientierten Unternehmen sind in Teltow, Wildau und Luckenwalde, auch ein Beleg dafür, dass die in diesen Städten etablierten Technologiezentren ihre Funktion sehr gut erfüllen. Unternehmen aus dem Cluster Gesundheitswirtschaft haben ihren Sitz schwerpunktmäßig in Teltow, Wildau und Luckenwalde. In Luckenwalde vor allem wegen des Biotechnologieparks. Unternehmen aus dem Cluster Verkehr, Mobilität, Logistik sind überwiegend in Wildau ansässig, viele dem Cluster Optik und Photonik zuzuordnende Unternehmen haben ihren Sitz in Teltow.

Die Verteilung der Unternehmen nach der Anzahl sagt allerdings noch nichts über deren wirtschaftliche Stärke (nach Umsatz und Beschäftigten) und/oder regionale Wirkung (z. B. hinsichtlich Steuern, Arbeitskräftebindung oder Kaufkraft) aus. Dies ist vor allem für die Standorte Ludwigsfelde und Blankenfelde-Mahlow wichtig, in denen einige große, technologieorientierte Unternehmen eine Niederlassung haben. Darüber hinaus ist eine hohe Dynamik im Bereich der technologieorientierten Unternehmen in der Flughafenumfeldregion zu verzeichnen, die im Zusammenhang mit der Umstellung der Automobilindustrie auf Elektromobilität steht und sowohl zu Ansiedlungen geführt hat, wie beispielsweise Tesla in Grünheide (vgl. Tesla, o. J.) und Microvast in Ludwigsfelde (vgl. Ludwigsfelde, o. J.), aber auch mit Arbeitsplatzabbau und Unternehmensverkauf/-verlagerung, wie im Luckenwalder SchaefflerWerk, verbunden sein kann (vgl. rbb, 2020). Auch die Covid-19-Pandemie hat zum Teil erhebliche Auswirkungen auf die Wirtschaft in der Flughafenumfeldregion. So ist der Flughafen BER unmittelbar 
selbst und wahrscheinlich über Jahre von der Pandemie betroffen, indirekt sind es auch die technologieorientierten Unternehmen, die in den Wertschöpfungsketten der Luftfahrtindustrie aktiv sind (vgl. MTU, 2020; Wangemann und Gellner, 2020).

Räumliche Nähe von Forschungseinrichtungen und innovativen Unternehmen spielt in Metropolregionen eine Rolle für die Innovativität von Unternehmen und Regionen. Diese These ist in der Literatur zur Raumforschung, zur Wissensökonomie oder Innovationsforschung verbreitet (vgl. z. B. Porter, 1998; Ache, 2000; Cooke 2001), wenn auch nicht unumstritten (vgl. Musil und Eder, 2017:95). In ihrer Untersuchung aus dem Jahr 2017 gehen Musil und Eder davon aus, dass die räumliche Nähe eine unterschiedliche Bedeutung für verschiedene Forschungsbereiche und Branchen hat (vgl. Musil und Eder, 2017:96). Die Forschungspolitik sollte daher fach- und branchenspezifische Standortanforderungen, beispielsweise bei Ansiedlungen, stärker berücksichtigen (vgl. Musli und Eder, 2017:93). Neben der Fachspezifik spielen nach Musil und Eder der Anteil und die Bedeutung impliziten Wissens in der Forschung eine Rolle für räumliche Nähe in Innovationsprozessen. Sie ist vor allem dann wichtig, wenn es sich um anwendungsorientierte Forschung handelt, wo der Anteil impliziten Wissens vergleichsweise hoch ist (vgl. Musil und Eder, 2017:96f).

Stimmt man diesen Thesen zu, ergibt sich für die Flughafenumfeldregion die Frage, ob die innovativen, technologieorientierten Unternehmen der Region auf ein zu ihren Anforderungen passendes wissenschaftliches Umfeld treffen und wie dieses ggf. gestärkt werden kann. Hierbei ist gleichwohl zu berücksichtigen, dass sich Forschungsinhalte und -methoden, auch anwendungsorientierter Forschung, nicht allein an den Anforderungen von Unternehmen ausrichten und schon gar nicht allein an den Anforderungen von Unternehmen aus der Region.

Ordnet man die für den Brandenburger Teil der Flughafenumfeldregion identifizierten Kompetenzen im Bereich der Forschung den Clustern der Wirtschaftsförderung zu, zeigt sich auf den ersten Blick folgendes: Bezogen auf das Cluster Gesundheitswirtschaft (27 Unternehmen) verfügt die TH Wildau mit dem Forschungsfeld angewandte Biowissenschaften, zu dem die Bereiche Bioprozesstechnik, Biosystemtechnik, Molekulare Biotechnologie und Funktionelle Genomik, High Performance Computing in Life Sciences, Diagnostische Bioinformatik und Mikrosystemtechnik/ Systemintegration gehören, über vielfältige Kompetenzen und Ressourcen. Hinzu kommen die Kompetenzen des Instituts für Biomaterialforschung (Helmholtz-Zentrum Geesthacht/Teltow) im Bereich multifunktionale, polymerbasierte Biomaterialien für Anwendungen in der Regenerativen Medizin. Mit der Ansiedlung des Bereichs Künstliche Intelligenz und Public Health des Robert Koch Instituts im Zentrum für Zukunftstechnologien in Wildau würde sich das Kompetenzspektrum noch erweitern. Die Arbeit des Life Sciences Scouts der TH Wildau im Rahmen des Projektes „Innovation Hub 13 - fast track to transfer“ unterstützt das Zustandekommen von Kooperationen und Projekten in diesem Cluster. 
Der Cluster IKT, Medien, Kreativwirtschaft (17 Unternehmen) trifft an der TH Wildau auf das Forschungsfeld Informatik/Telematik mit den folgenden Kompetenzfeldern:

- Business Intelligence

- Decision Support Systeme

- Gebäudetelematik

- Mobile Informations- und Assistenzsysteme

- Softwareentwicklung / Software Engineering

- Telematik

- Verkehrstelematik

- Verwaltungsinformatik und digitale Medien

Auch dieser Cluster wird seitens der TH Wildau mit einem Scout für digitale Integration unterstützt. Die einzelnen Kompetenzfelder sind bisher jedoch noch wenig integriert.

Im Zusammenhang mit dem Cluster Metall (13) steht an der TH Wildau das Forschungsfeld Produktion und Material mit den Themen iC3@Smart Production, Maschinendynamik und lärmarme Konstruktion sowie Werkstofftechnik, Metallische Werkstoffe und Analytik. Die beabsichtigte Ansiedlung eines Bereiches des Fraunhofer-Institutes für Produktionsanlagen und Konstruktionstechnik in der Flughafenumfeldregion würde die Kompetenzen in diesem Bereich wesentlich verstärken.

Auch Unternehmen aus dem Cluster Verkehr, Mobilität, Logistik (12) treffen auf ein breites Kompetenzspektrum an der TH Wildau. Das reicht vom Aviationmanagement /Aviation Security über Luftfahrttechnik (insbesondere unbemannte Kleinflugzeuge), Verkehrslogistik (insbesondere Lagerlogistik, Makrologistik, City Logistik, Logistik für Forst- und Holzwirtschaft) bis hin zur sicheren Objektidentität (insbesondere Prozess- und Systeminnovation/Industrie 4.0, Digitalisierung, IoT; Auto ID/RFIDTestzentrum). In all diesen Bereichen sind Forschungsgruppen aktiv.

Mit Bezügen zum Cluster Kunststoff und Chemie (11) wird an der TH Wildau an polymeren Hochleistungsmaterialien und Faserverbund-Materialtechnologien (Entwicklung von Harzsystemen und Funktionsintegration bei Compositen) gearbeitet. Auch das Fraunhofer IAP, FB PYCO forscht am Standort zu Polymermaterialien und Composites. Hier zeigt sich ein vergleichsweise hoher Spezialisierungsgrad bezogen auf ein Cluster.

Im Brandenburger Teil der Flughafenumfeldregion sind relativ wenige innovative, technologieorientierte Unternehmen in den Clustern Energietechnik (7), Optik und Photonik (8), Automatisierungstechnik, Chips, Sensoren (7) sowie Umwelttechnik (3) aktiv. Bezogen auf das Cluster Optik und Photonik 
wird an der TH Wildau seit vielen Jahren erfolgreich im Forschungsfeld Photonik, Laser- und Plasmatechnologien gearbeitet, das breit aufgestellt ist. Kompetenzfelder bilden im Forschungsfeld Materialsynthese und -untersuchungen, die Herstellung und Charakterisierung optoelektronischer Bauteile und Komponenten sowie Prozesscharakterisierung und Optimierung. Darüber hinaus sind an der TH Wildau Kompetenzen vorhanden, die passfähig zu den übrigen Clustern sind. Sie sind allerdings weniger stark gebündelt und mit personellen Ressourcen untersetzt.

\subsection{Ermittlung der Perspektive ausgewählter Akteur/innen}

Um den Status quo der Flughafenumfeldregion aus der Sicht unterschiedlicher Regionalakteur/innen kennenzulernen, wurden sieben leitfadengestützte Interviews mit 12 Expert/innen geführt. Drei Personen kommen aus dem Bereich Wissenschaft und Forschung (Robert Koch Institut und FraunhoferInstitut für Produktionsanlagen und Konstruktionstechnik), sieben aus der Wirtschaftspolitik bzw. Wirtschaftsförderung (Ministerium für Wirtschaft, Arbeit und Energie des Landes Brandenburg, Wirtschaftsförderung Land Brandenburg $\mathrm{GmbH}$, Wirtschaftsförderungsgesellschaft Dahme-Spreewald $\mathrm{mbH}$ ) und zwei aus Unternehmen (Stadt- und Überlandwerke GmbH Lübben sowie Flughafen BerlinBrandenburg GmbH). Die Interviews fanden im Zeitraum September bis November 2020 statt.

Im Folgenden werden die Interviews nach den Themenbereichen „Räumliche Dimension/Erreichbarkeit", „Zusammenarbeit der Akteur/innen“, „Gewerbeflächen/Ansiedlung" , „Innovationsthemen“ und „Hemmnisse“ ausgewertet.

\section{Räumliche Dimension/Erreichbarkeit der Flughafenumfeldregion}

In den Interviews wurde deutlich, dass es unterschiedliche Perspektiven auf die Flughafenumfeldregion gibt, die mit einem spezifischen räumlichen Verständnis zusammenhängen. Unterschieden werden können die Perspektive der Wirtschaftsförderung, der Immobilienentwicklung und der Raumplanung/Regionalentwicklung. Die Perspektive der Wirtschaftsförderung geht mit einem differenzierten Regionenverständnis einher. Zu nennen ist zunächst eine Erweiterung der ursprünglichen Gebietskulisse des GSK, z. B. im Zusammenhang mit der Ansiedlung von Tesla in Grünheide. Eine stark ausgeweitete Sicht auf die Gebietskulisse der Flughafenumfeldregion bezieht ganz Südbrandenburg im Dreieck Berlin, Dresden, Leipzig ein. Aus der Sicht der Wirtschaftsförderung spielt aber auch das engere Flughafenumfeld eine Rolle, etwa wenn es um die Ansiedlung von Unternehmen aus Branchen mit internationaler Reisetätigkeit geht, für die eine schnelle Erreichbarkeit des Flughafens wichtig ist. Auch für ansiedlungsinteressierte Forschungseinrichtungen kann dieses Kriterium von Bedeutung sein. 
Die Immobilienentwicklungsperspektive fokussiert auf den Flughafen selbst bzw. sein enges räumliches Umfeld und die Entwicklungsachsen nach Berlin hinein.

In der Perspektive der Raumplanung sind funktionale Teilräume und Entwicklungsachsen, die auch in dem der Analyse zugrundeliegenden Verständnis eine Rolle spielen, wichtig. In diesem Zusammenhang wurde von einigen Expert/innen betont, dass die räumlich begrenzten Wachstumspotentiale Berlins zur Notwendigkeit der Ausweitung nach Südbrandenburg führen. Dies beträfe ganz besonders den immer noch schnell wachsenden Standort Adlershof. Sichtbar wird das an den so genannten Innovationskorridoren, die aus Berlin hinaus in den Brandenburger Teil der Flughafenumfeldregion führen bzw. sich entlang des Autobahnringes entwickeln.

Was die Unterscheidung zwischen dem Berliner und dem Brandenburger Teil der Flughafenumfeldregion angeht, wurde darauf verwiesen, dass diese durch sehr unterschiedliche Entwicklungsbedingungen und Entwicklungskontexte gekennzeichnet sind, so dass die entsprechenden Akteur/innen auch unterschiedliche Sichtweisen auf die Flughafenumfeldregion haben. Häufig würde sie von Berliner Akteur/innen gar nicht als Flughafenumfeldregion wahrgenommen, die Zugehörigkeit zur Metropole ist für die Akteur/innen viel wichtiger. Die geführten Interviews bestätigen die Definition der Gebietskulisse, wie sie vom Analyseteam vorgenommen wurde (vgl. Darstellung 3 und Darstellung 4).

\section{Zusammenarbeit der Akteur/innen}

Die Interviewteilnehmer/innen waren sich darüber einig, dass die Zusammenarbeit der Akteur/innen in der Region stark verbesserungswürdig ist. Es überwögen vereinzelte, meist kleine, Kommunen mit kleinteiligen Sichtweisen und Interessen, die zum Teil keine anspruchsvollen Entwicklungsziele verfolgten. Es sei kein Gesamtverständnis für die Region vorhanden und auch keine längerfristige Entwicklungsstrategie erkennbar.

Positiv gestaltet sich eine beginnende Zusammenarbeit zwischen dem Ministerium für Wirtschaft, Arbeit und Energie des Landes Brandenburg (MWAE), der Gemeinsamen Landeplanung, den Regionalen Wachstumskernen, den Landkreisen, dem Flughafenmanagement und dem Dialogforum bezüglich der Vermarktung der Gewerbeflächen. Diese positive Meinung wurde jedoch zum Teil auch wieder relativiert.

Auch die Zusammenarbeit zwischen den beiden Bundesländern Berlin und Brandenburg sowie zwischen den Berliner Stadtbezirken der Flughafenumfeldregion sowie den Brandenburger Kommunen wurde als ausbaufähig eingeschätzt. Erwähnung fand auch die Notwendigkeit einer verbesserten Zusammenarbeit zwischen öffentlichen und privaten Akteur/innen. 
Eine wichtige Voraussetzung für die Verbesserung der Zusammenarbeit wird im Aufbau einer professionellen Organisationsstruktur für die Region gesehen.

\section{Gewerbeflächen/Ansiedlung}

Bedauert wurde von den Interviewten eine weitgehend ungesteuerte Ansiedlung in der Flughafenumfeldregion, im Unterschied beispielsweise zur Umfeldregion des Flughafens Amsterdam. Es wurde zwar versucht, die dort gemachten Erfahrungen und Impulse zu nutzen, jedoch mit geringem Erfolg. Inzwischen haben sich überproportional Logistikunternehmen angesiedelt, wobei der Standort von Amazon in Kienberg als negatives Beispiel genannt wird, das auch noch mit unerwünschten Verkehrsbelastungen einhergeht. Mit Ausnahme der Güterverkehrszentren sei keine Profilierung erkennbar. Hiermit im Zusammenhang stehen nach Auffassung einiger Expert/innen steigende Immobilienpreise aufgrund der Vermarktungsinteressen privater Eigentümer.

In den Gesprächen wurde darauf verwiesen, dass entsprechend dem aktuellen Gewerbeflächenkonzept Flughafenumfeld BER 136 Standorte mit einer Gesamtfläche von 3.941 Hektar vorhanden sind, wovon allerdings bereits 86 Prozent vermarktet sind. Lediglich 27,5 Prozent der Flächen sind im Besitz der öffentlichen Hand, was eine gesteuerte Gewerbeflächenentwicklung und -nutzung erschwert. Verfügbar sind nach Abzug der Flächen für den Teslastandort in Grünheide (310 ha) noch etwa 240 ha wobei es sich um keine großen zusammenhängenden Flächen handelt. Größere und gut erreichbare Flächen finden sich nach Aussage der Experten noch in Ragow und Mittenwalde.

Was das Potenzial an Flächen für den Wohnungsbau betrifft, verwies ein Experte auf die Notwendigkeit einer qualitativ hochwertigen Verdichtung in einzelnen Kommunen, die allerdings nicht immer von den Kommunen so gesehen wird.

Im Eingangsbereich des Flughafens zeichnet sich ein Trend zu Bürogebäuden ab, ähnlich wie in der DownTown-Achse nach Berlin mit der BRAIN BOX Berlin.

Mehrere Interviewpartner halten es für sinnvoll, im Umfeld des Flughafens einen stärkeren Wissenschafts- und Technologiestandort aufbauen. Hierfür ist nach Auffassung der Interviewten eine kritische Masse vorhanden, der Arbeitsmarkt in Berlin kann erschlossen werden und Fördermöglichkeiten bestehen über Mittel aus dem Strukturstärkungsgesetz. Hierbei ist zu berücksichtigen, dass die Ansiedlung einer außeruniversitären Forschungseinrichtung die räumliche Anbindung an eine Hochschule und die Nähe zur Industrie erfordert. Auch die Erschließung von Standorten, die vom Flughafen weiter entfernt sind, wird als eine Option der Stärkung des Wissenschaftspotenzials der Flughafenumfeldregion verfolgt. Hierfür könnte die Zusammenarbeit zwischen Lübben und dem Standort Adlershof als 
Modell entwickelt werden. Geplant ist die Schaffung größerer Coworking-Kapazitäten für Mitarbeiter/innen von Unternehmen und Forschungseinrichtungen in Adlershof die in der Region wohnen bzw. dort Ansiedlungsmöglichkeiten finden sollen.

\section{Innovationsthemen}

Innovationsthemen wurden den Expert/innen für die Bereiche Gesundheit, Digitalisierung, Infrastruktur und Mobilität/Verkehr thematisiert.

Im Zusammenhang mit der geplanten Ansiedlung einer Arbeitsgruppe des Robert Koch Instituts im Zentrum für Zukunftstechnologien in Wildau, wurden als wichtige Innovationsfelder Infektionskrankheiten und Pandemieplanung, Antibiotikaresistenz und Krankheitsprävention genannt, deren erfolgreiche Bearbeitung nicht nur inter- sondern auch transdisziplinäre Ansätze erfordert. Eine zunehmende Bedeutung hätten auch Big Data Analysen auf Populationsebene. Angesichts der gegenwärtigen Covid-19-Pandemie würde das RKI Ansätze zur Veränderung der Ausatemluft im Falle von Infektionen sowie zur gezielten Identifizierung und Bekämpfung von Aerosolen in Räumen begrüßen. Die gegenwärtige Situation verweist darauf, dass es neuer Formate der Wissenschaftskommunikation bedarf und das Führen öffentlicher Diskurse durch die Wissenschaft stärker unterstützt werden sollte.

Was das breite Feld der Digitalisierung betrifft, wurde auf die Notwendigkeit der Optimierung von Verwaltungsabläufen mittels digitaler Technologien verwiesen und als Beispiel der Teilbereich der Beschaffung genannt, der durch den Einsatz von Künstliche Intelligenz effizienter gestaltet werden und den Gegenstand einer Kooperation zwischen dem RKI und der TH Wildau bilden könnte.

Von einem anderen Experten wurde der Einsatz von VR/AR-Technologien in Unternehmen der Luftfahrttechnik als Innovationsthema der Digitalisierung benannt. Auch könne Crowd Production ein Innovationsansatz sein, in dem Unternehmen gemeinsam kollaborativ innovieren und eine Wertschöpfungskette aufbauen, um dann als Systemanbieter fungieren zu können.

Besonderen Raum nahm das Innovationsmodell der Stadt- und Überlandwerke GmbH Lübben mit seinen facettenreichen Innovationskomponenten ein. Zu denen gehört (1) die Schaffung von Coworkingspace für Wissenschaftler/innen, (2) das Angebot anspruchsvoller Wohnmöglichkeiten inklusive energieautarkem Wohnen, Miet-Flat und eCar-Sharing, (3) die Umstellung des ÖPNV auf Brennstoffzellenbusse, die mit selbst erzeugtem grünen Wasserstoff betrieben werden, (4) der Aufbau eines eigenen Glasfasernetzes für den 5G-Betrieb und das autonome Fahren im ländlichen Raum sowie der Bau eines eigenen Gasnetzes. 


\section{Hemmnisse}

Mehrere der befragten Expert/innen gehen davon aus, dass sich bereits heute problematische Verkehrssituationen in der Region weiter zuspitzen werden, wenn der Flughafen BER nach der Pandemie in einen normalen Betriebsmodus übergehen wird. Das aktuelle Verkehrsgutachten von Spreeplan Verkehr GmbH aus dem Jahr 2019 geht insbesondere von stark belasteten Knoten im Individualverkehr entlang der B96a bei Großbeeren, Blankenfelde und an den Anschlussstellen Schönefeld Nord und Süd aus. Darüber hinaus wird der „IKEA-Knoten Waltersdorf" ebenfalls als bereits stark ausgelastet bewertet (vgl. SPV, 2019:29).

Ein nicht ausreichendes Wohnungsangebot im Brandenburger Teil der Flughafenumfeldregion bildet den zweiten Problembereich, der in den Interviews genannt wurde. Die Einschätzungen der Interviewten stimmen mit den Ergebnissen der Analyse zu den Angeboten in den Gemeindesteckbriefen überein.

Erwähnung fanden die spürbaren wirtschaftlichen Probleme der Luftfahrt- und Automobilindustrie infolge der Corona-Pandemie, die gegenwärtig wie ein retardierendes Moment für die Entwicklung der Gesamtregion wirken. Auch und gerade der Flughafen selbst sieht sich wirtschaftlichen Problemen gegenüber, für die ein Ende momentan nicht absehbar ist, was beispielsweise die Immobilienentwicklung des Flughafens hemmt. Es wurde auch die Vermutung geäußert, dass sich ein Reiseverhalten wie vor der Pandemie nicht wieder einstellen wird.

Kulturelle Offenheit und Toleranz sowie ehrgeizige Anspruchshaltungen der Kommunen wurden als notwendige Voraussetzung für eine erfolgreiche Regionalentwicklung ebenso hervorgehoben wie ein professionelles, Kommunen übergreifendes, Regionalmanagement.

\subsection{Good Practice Beispiele}

Um die Erfahrungen anderer Flughafenregionen zu berücksichtigen und diese in zukünftige Diskussionen in der Region des BER einzubringen, wurde nach Good Practice Beispielen gesucht. Als geeignet hierfür erwiesen sich die "Guidelines for Decion Makers - Sustainable Airport Areas" aus dem Jahr 2018 (vgl. IAU, 2018). Sie entstanden im Ergebnis eines mehrjährigen internationalen Diskussionsprozesses, der mehrere Workshops umfasste und von der Metropolis Initiative on Sustainable Airport Areas angestoßen wurde. Der initiative gehörten folgende Partner an:

- The Paris Region Council (Conseil Régional d'Ile-de-France)

- Hubstart Paris Region Association

- $\quad$ ARC (Atlanta Regional Commission) 
- ARC (Airport Regions Conference)

- GIP Emploi Roissy CDG

- Orly International

- Paris Aéroport

- AMB (Àrea Metropolitana de Barcelona)

- Paris Region Entreprises (vgI. IAU, 2018:2).

Am ersten Workshop in Paris im Jahr 2015 beteiligten sich mehr als dreißig Expert/innen aus acht internationalen Flughafenregionen. Am zweiten Workshop in Atlanta nahmen Vertreter/innen von sechs internationalen Flughäfen (Atlanta, Paris-CDG, Beijing, Dakar, Vantaa, and Barcelona) sowie internationale Expert/innen und Wissenschaftler/innen aus Paris, Atlanta, Hong-Kong and Vancouver teil. Sie identifizierten Good Practices in den Bereichen Bereichen Mobilität/Erreichbarkeit, Stadtplanung, Governance und Wettbewerbsfähigkeit (vgI. IAU, 2018:6). Der dritte und abschließende Workshop fand im Jahr 2017 in Paris statt. Er wurde gemeinsam mit der Airport Regions Conference (ARC) organisiert. An diesem nahmen 35 Teilnehmer/innen aus 13 Metropolen und Flughafenregionen (Barcelona, Berlin, Brussels, Budapest, Daegu, Dakar, Dublin, Goteborg, Oslo, Paris-CDG, Paris-Orly, Vantaa and Warsaw) sowie internationale Expert/innen und Berater/innen teil. Dieser Workshop hatte die Themen Umwelt, Mobilität und Erreichbarkeit, Ökonomische Entwicklung sowie Stadtplanung zum Schwerpunkt (vgl. IAU, 2017:7). Im Ergebnis dieses Prozesses entstanden die weiter o. g. Guidelines, die als eine mit hoher Expertise erarbeitete und aktuelle Good Practice Zusammenstellung betrachtet werden können. Auf sie wurde daher in der Analyse zurückgegriffen.

In den Guidelines systematisierten die Autor/innen die wichtigsten Herausforderungen für Flughafenregionen (vgl. Darstellung 18), beschrieben sie und untersetzten sie mit Beispielen. Vor dem Hintergrund des aktuellen Entwicklungsstandes der Umfeldregion des Flughafens BER wurde einige dieser Beispiele als Good Practice Beispiele ausgewählt und zusammenfassend beschrieben. Im Folgenden sind drei Beispiele dargestellt, weitere finden sich in der Anlage 2. 


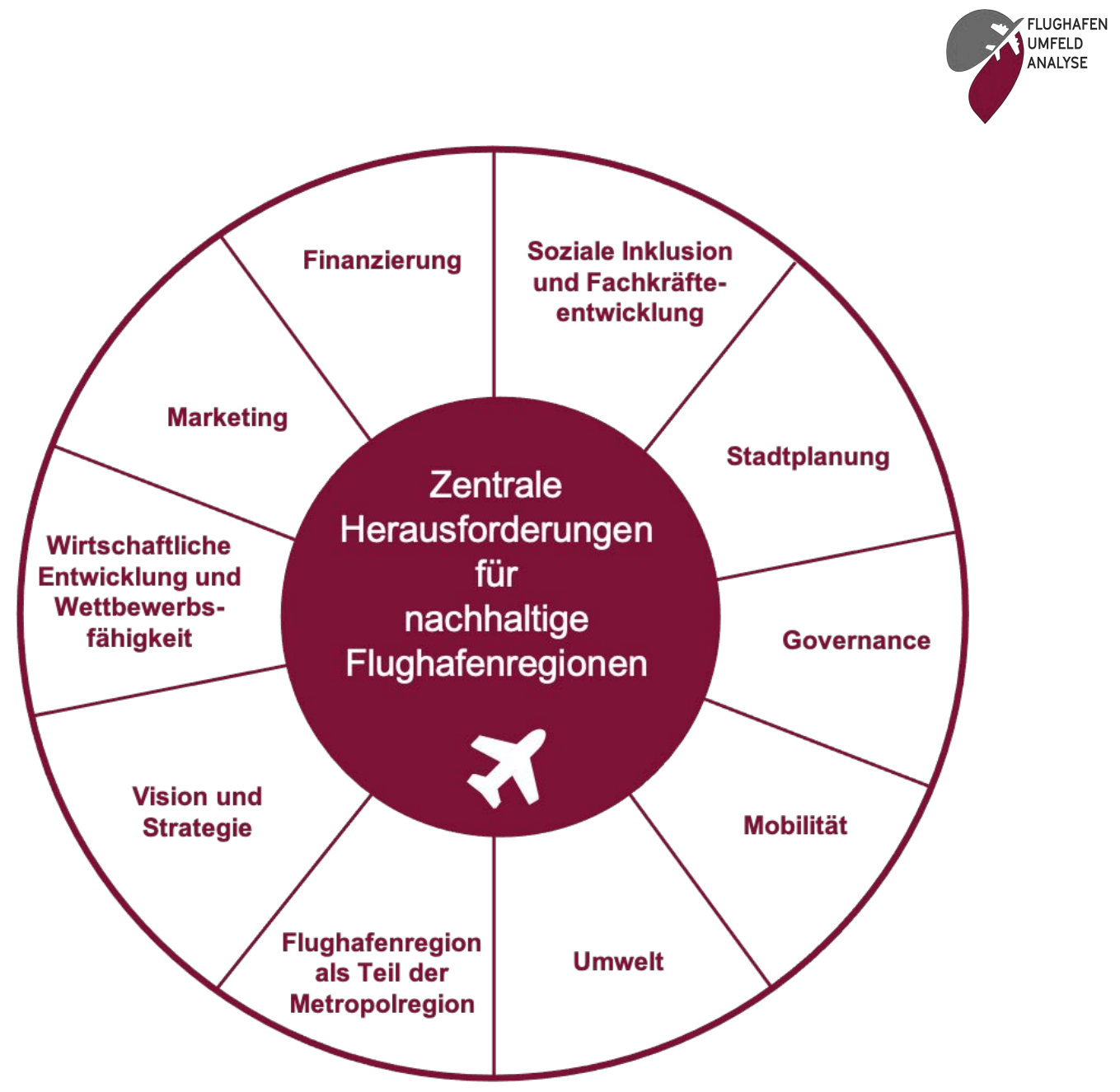

Darstellung 18: Herausforderungen für nachhaltige Flughafenregionen (IAU, 2018:5)

Das erste Beispiel ist auf eine flexible und dynamische Ansiedlungsplanung im Umfeld des HartsfieldsJackson Atlanta International Airport gerichtet. Hier wurde die Ansiedlung eines neuen Hauptsitzes von Porsche durch den Staat Georgia und die Stadt Atlanta unterstützt (vgl. Darstellung 20). „The importance of being open to new opportunities arising: The arrival of Porsche in the airport area of Hartfield-Jackson Atlanta International Airport is an "accident of history" made possible thanks to the flexibility of the urban planning" (IAU, 2018:23). 


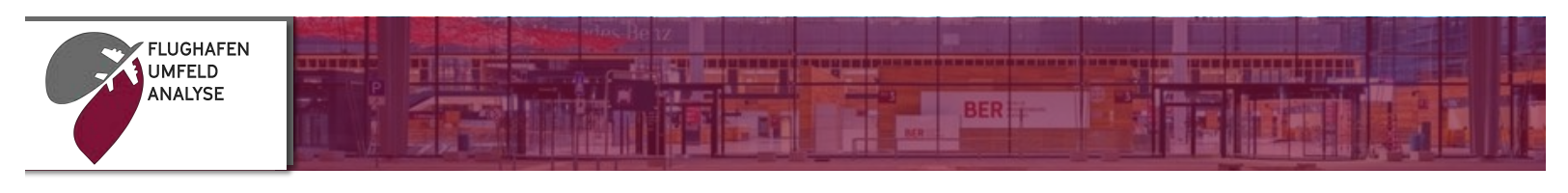

Good Practice Beispiel für flexible und dynamische Ansiedlungsplanung in einer Flughafenregion

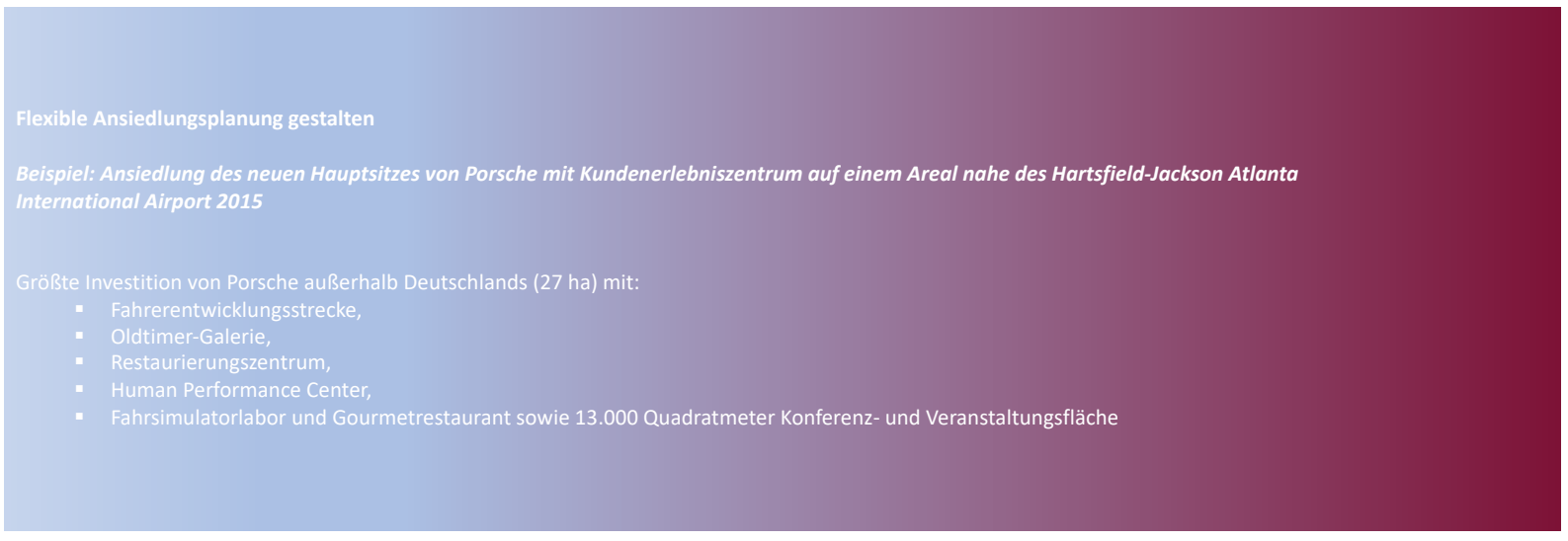

Darstellung 19: Good Practice Beispiel Ansiedlungsplanung (IAU, 2018:23)

Das zweite Beispiel verweist auf die Entwicklung eines kompakten, innovativen Standortes auf einem Flughafen durch die Zusammenarbeit zahlreicher Akteur/innen aus der Wissenschaft, der Wirtschaft und dem Bereich der Immobilienentwicklung. Im Zentrum stehen das kollaborative Entwickeln, Testen und Umsetzen von Innovationen in neuen räumlichen Settings (vgl. Darstellung 20). Hierzu gehört auch der Betrieb eines Testlabs, in dem Unternehmen Produktideen und Prototypen testen lassen können.

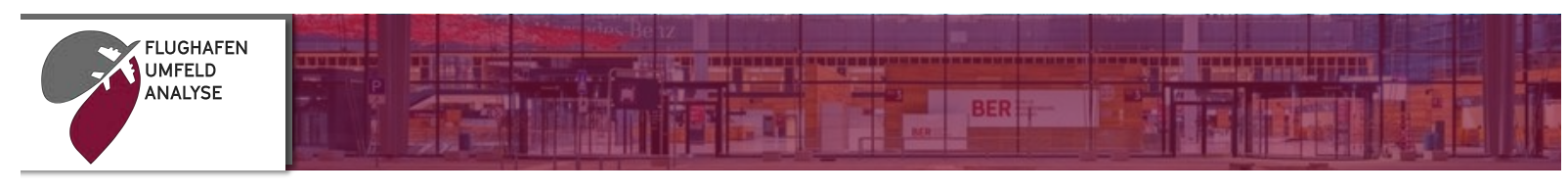

Good Practice Beispiel für Innovation und Flughafenregion

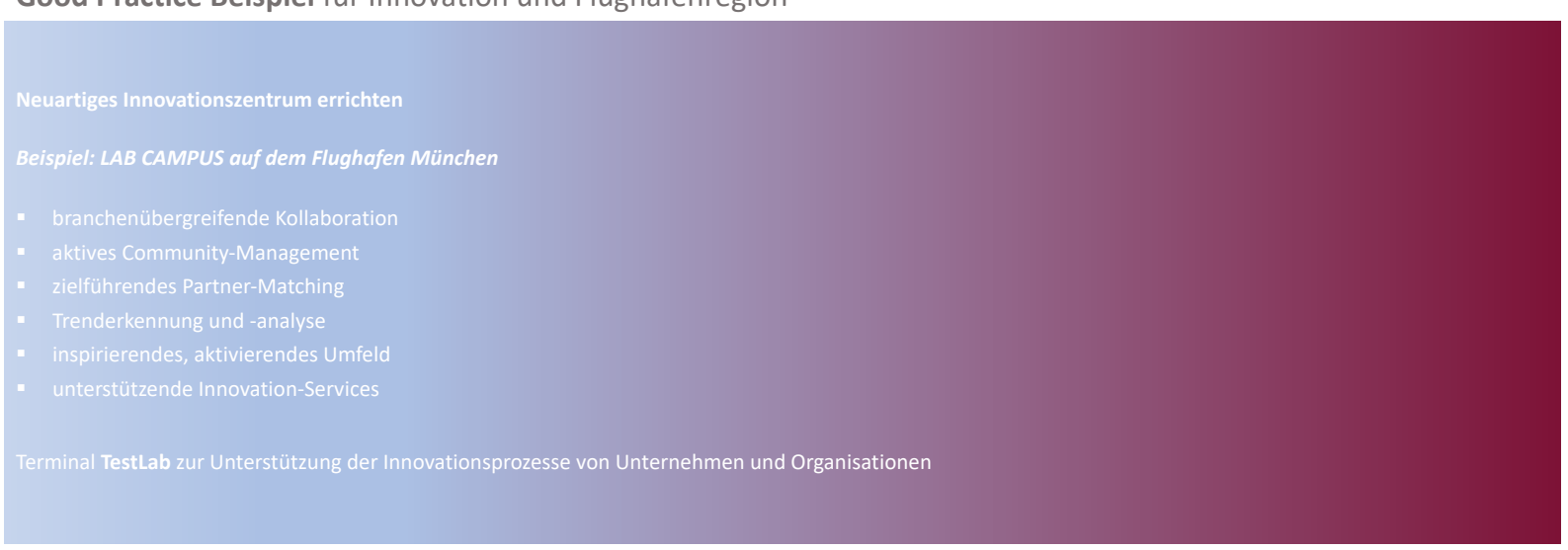

Darstellung 20: Good Practice Beispiel Innovationsstandort (LABCampus, 2020) 
Das dritte Beispiel ist dem Bereich nachhaltige Mobilitätslösungen zugeordnet. Bereits vor etlichen Jahren sah sich der Flughafen Arlanda der Problematik gegenüber, einen Betrag zur Begrenzung der $\mathrm{CO}_{2}$-Emissionen von Stockholm zu leisten. Es wurde ein Modell für den stärkeren Einsatz von Öko-Taxis entwickelt und erfolgreich umgesetzt. Das Modell wurde auch auf weitere Flughäfen übertragen (vgl. Darstellung 21).

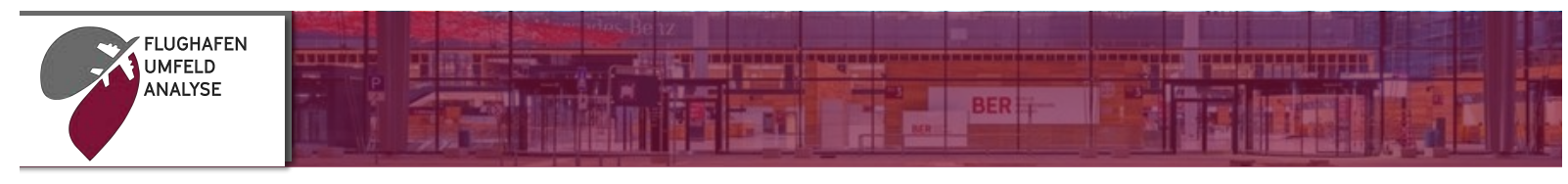

Good Practice Beispiel für Mobilitätslösungen in einer Flughafenregion

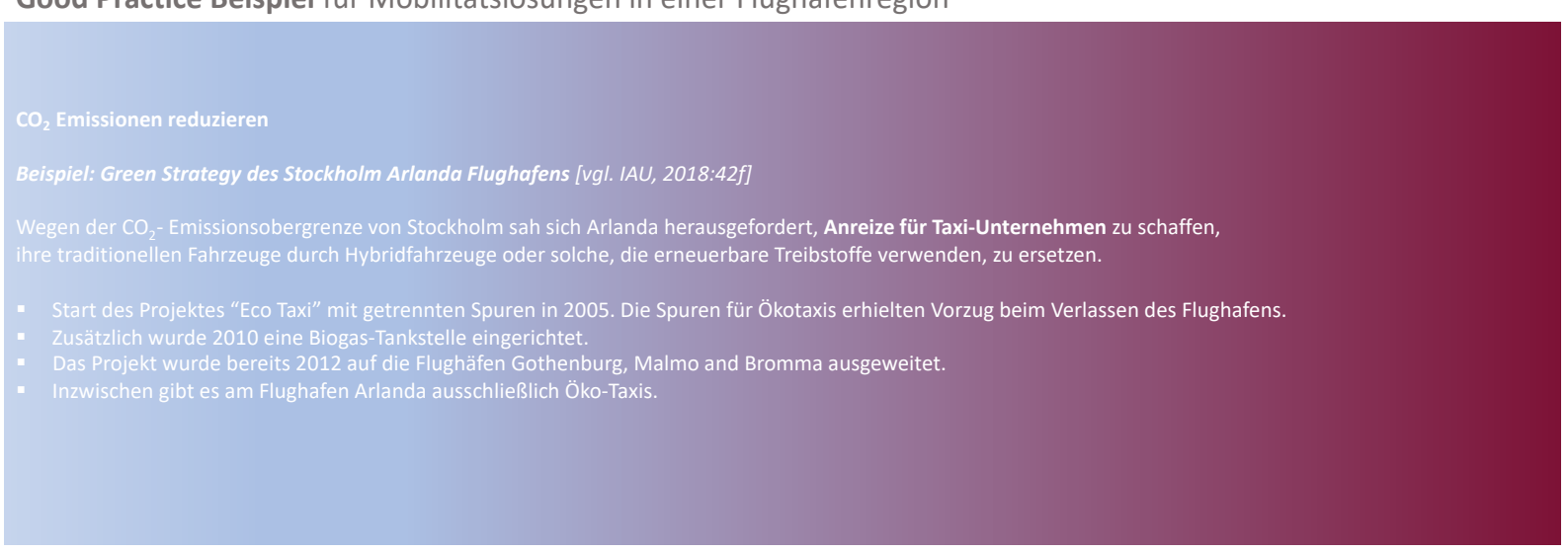

Darstellung 21: Good Practice Beispiel für Mobilitätslösungen (IAU, 2018:42 f.)

\subsection{Handlungsansätze}

Basierend auf den Ergebnissen der bisher dargestellten Arbeitsschritte hat das Untersuchungsteam Handlungsansätze abgeleitet, die auf dem digitalen Forum „Die Rolle von Wissenschaft und Innovation in der Flughafenumfeldregion - Herausforderungen und Perspektiven“ im Dezember vorgestellt und diskutiert wurden. Sie werden nachfolgend beschrieben und im Anschluss in einer Matrix unterschiedlichen Akteur/innen zugeordnet, die diese aufgreifen, operationalisieren und umsetzen können. In der Regel sind diese Handlungsansätze für mehrere Akteur/innen gleichzeitig relevant und können nur gemeinsam weiterentwickelt und umgesetzt werden.

(1) Ausbau des Wissenschafts- und Technologiestandortes Wildau durch die Ansiedlung weiterer Wissenschaftseinrichtungen im engeren Flughafenumfeld und mit Nähe zur TH Wildau sowie Stärkung seiner Funktion als Kooperationsknoten und Mittler zwischen den Berliner und Lausitzer Wissenschafts- und Technologiepotenzialen. 
(2) Standort und Teilregionen übergreifende, kooperative Entwicklung komplexer Innovationsfelder, basierend auf den spezifischen Kompetenzen unterschiedlicher Akteur/innen.

(3) Unterstützung der Entwicklung von Lübben als neuen Wissenschafts- und Technologiestandort in der Flughafenumfeldregion durch die Schaffung von Coworking-Möglichkeiten sowie geeigneter Ansiedlungsbedingungen für Personal aus Wissenschaftseinrichtungen und Unternehmen des Standortes Adlershof sowie den Aufbau neuer Kooperationsbeziehungen in der Flughafenumfeldregion.

(4) Ausbau der Zusammenarbeit mit Immobilienentwicklern bezüglich geeigneter Ansiedlungsbedingungen für Wissenschaftseinrichtungen, innovative Unternehmen und Startups. Diese beziehen sich auf räumliche Settings und Innovationsformate.

(5) Ausbau der Zusammenarbeit der Wissenschaftseinrichtungen mit dem Flughafen hinsichtlich seiner Profilierung als Testfeld für innovative Produkte und Dienstleistungen, als Raum der Wissenschaftskommunikation sowie als Schaufenster für Wissenschaft und Technologie der Metropolregion.

(6) Stärkere Bündelung der Wissenschaftskompetenzen der Europa-Universität Viadrina, der FH Potsdam und der TH Wildau an den Präsenzstellen und Profilierung der Präsenzstellen als Transferplattformen der Flughafenregion. Einbindung von Flughafenumfeldkommunen in die Arbeit der Präsenzstellen.

(7) Stärkerer Einbezug von Akteur/innen aus der Wissenschaft in die Strategie- und Planungsprozesse der Region (z. B. GSK, Dialogforum).

(8) Entwicklung einer gemeinsamen Vision der Flughafenumfeldregion mit Methoden der strategischen Vorausschau.

(9) Unterstützung der Bürgerbeteiligung mit Methoden und Formaten von Citizen Science.

Mit der Umsetzung und Spezifizierung der Handlungsansätze werden insbesondere die in der nachfolgenden Darstellung angesprochenen Akteur/innen adressiert. 


\begin{tabular}{|c|c|c|c|c|c|}
\hline Handlungsansatz & Wissenschaft & Wirtschaft & Politik & $\begin{array}{l}\text { Wirt- } \\
\text { schafts- } \\
\text { förderung }\end{array}$ & Kommunen \\
\hline 1. Kooperationsknoten & THWi & $\begin{array}{l}\text { Wirtschaftsre- } \\
\text { gion Lausitz } \\
\text { GmbH }\end{array}$ & MWFK & $\begin{array}{l}\text { WFBB, } \\
\text { WFG LDS }\end{array}$ & $\begin{array}{l}\text { RWK Schönefelder } \\
\text { Kreuz }\end{array}$ \\
\hline 2. Innovationsfelder & $\begin{array}{l}\text { Alle Wissen- } \\
\text { schaftseinrich- } \\
\text { tungen der } \\
\text { FUR }\end{array}$ & $\begin{array}{l}\text { Wirtschaftsre- } \\
\text { gion Lausitz } \\
\text { GmbH }\end{array}$ & $\begin{array}{l}\text { MWFK und } \\
\text { MWAE }\end{array}$ & WFBB & \\
\hline 3. Lübben & THWi & $\begin{array}{l}\text { Stadtwerke Lüb- } \\
\text { ben, WISTA Ma- } \\
\text { nagement }\end{array}$ & MWAE & WFBB & Stadt Lübben \\
\hline $\begin{array}{l}\text { 4. Ansiedlungs- } \\
\text { bedingungen }\end{array}$ & THWi & $\begin{array}{l}\text { Immobilienwirt- } \\
\text { schaft, FBB }\end{array}$ & & WFG LDS & $\begin{array}{l}\text { RWK Schönefelder } \\
\text { Kreuz }\end{array}$ \\
\hline 5. Schaufenster & THWi & $\begin{array}{l}\text { FBB, WISTA Ma- } \\
\text { nagement, Im- } \\
\text { mobilienwirt- } \\
\text { schaft }\end{array}$ & & & \\
\hline 6. Präsenzstellen & $\begin{array}{l}\text { THWi, Vi- } \\
\text { adrina, FH } \\
\text { Potsdam }\end{array}$ & & MWFK & & $\begin{array}{l}\text { RWK Fürstenwalde, } \\
\text { Luckenwalde, FUR- } \\
\text { Kommunen }\end{array}$ \\
\hline 7. Strategiebildung & THWi & $\begin{array}{l}\text { Regionalma- } \\
\text { nagement FUR }\end{array}$ & & WFBB & $\begin{array}{l}\text { Dialogforum, Lu- } \\
\text { ckenwalde, Lud- } \\
\text { wigsfelde, Fürsten- } \\
\text { walde, Lübben }\end{array}$ \\
\hline 8. Vision & THWi & $\begin{array}{l}\text { Regionalma- } \\
\text { nagement FUR }\end{array}$ & & & $\begin{array}{l}\text { Dialogforum, Lu- } \\
\text { ckenwalde, Fürs- } \\
\text { tenwalde, Lübben }\end{array}$ \\
\hline 9. Bürgerbeteiligung & THWi & & & & $\begin{array}{l}\text { Dialogforum, Lu- } \\
\text { ckenwalde, Fürs- } \\
\text { tenwalde, Lübben }\end{array}$ \\
\hline
\end{tabular}

Darstellung 22: Adressierte Akteure/innen für die Operationalisierung und Umsetzung der Handlungsoptionen

\section{Das digitale Forum „Die Rolle von Wissenschaft und Innovation in der Flughafenumfeldregion - Herausforderungen und Perspektiven“}

Am digitalen Forum, das am 03. Dezember 2020 als Onlinekonferenz stattfand, beteiligten sich mehr als 50 interessierte Personen aus Wissenschaft, Wirtschaft und Politik (vgl. Programm in Anlage 3).

Neben der Präsentation der keynote, mit der die Hauptergebnisse der Analyse zur Flughafenumfeldregion vorgestellt wurden, fand eine Diskussion im Podium statt, an der sich der Staatssekretär des MWFK des Landes Brandenburg, Tobias Dünow, der stellvertretende Präsident des RKI, Prof. Dr. med. Lars Schaade, der Geschäftsführer der Wirtschaftsförderung Brandenburg, Sebastian Saule, der Direktor für den Bereich Astroteilchenphysik am DESY, Prof. Dr. Christian Stegmann sowie der Vizepräsident für Forschung und Transfer der TH Wildau, Prof. Dr. Klaus-Martin Melzer, beteiligten. 
Mit der Frage nach der Zusammenarbeit zwischen den Ländern Berlin und Brandenburg wurde die Diskussion eröffnet. Sowohl seitens des Staatssekretärs aus dem MWFK als auch des Geschäftsführers der Wirtschaftsförderung Brandenburg wurde die Zusammenarbeit bezüglich Wissenschaft und Innovation als sehr gut bewertet. Als Beispiele hierfür wurden gemeinsame Berufungsverfahren und die gemeinsame Innovationsstrategie der Länder hervorgehoben. Verbesserungswürdig sei die Kommunikation der erfolgreichen Zusammenarbeit sowie die Darstellung der Region als Wissenschaftsstandort. Einen weiteren Diskussionsschwerpunkt bildete die Frage, ob der Wissenschafts- und Technologiestandort Wildau die Rolle eines Kooperationsknotens zwischen Akteur/innen aus dem Berliner Teil der FUR und denen aus der Lausitz spielen kann. Für die positive Beantwortung dieser Frage wurden zur Begründung die bisherigen Arbeiten im Rahmen des Innovation Hub 13 Projekts angeführt sowie Aktivitäten im Zusammenhang mit der Ansiedlung von Wissenschaftler/innen aus Adlershof am Standort Lübben. Es konnte herausgearbeitet werden, dass für eine solche Entwicklung multidimensionale Perspektiven erforderlich seien und man diesbezüglich auf einem guten Weg sei. Einen dritten Diskussionsschwerpunkt bildete das Ansiedlungsgeschehen in der Region. Dargestellt wurde die Zusammenarbeit zwischen der Wirtschaftsförderung auf Landeseben mit der auf der Ebene der Landkreise und Kommunen. Die zunehmende Bedeutung der Verfügbarkeit geeigneter Fachkräfte als Ansiedlungskriterium für Unternehmen wurde betont. Möglicherweise spielt auch die Verfügbarkeit von Wissenschaftseinrichtungen, deren Nutzung als Standortvorteil immer wieder geprüft werden müsse, eine Rolle bei der Ansiedlung von Unternehmen. Wünschenswert sei eine stärkere Orientierung auf Ansiedlungsaktivitäten an einem Leitbild der Flughafenumfeldregion. Damit könnte das Gewerbeflächenkonzept der FUR auch besser in das in der Entwicklung befindliche Gewerbeflächenkonzept für das gesamte Land Brandenburg integriert werden. Die Vertreter/innen der Wissenschaft stellten dar, wie die Bearbeitung einzelner Forschungsthemen zu neuen regionalen Konstellationen von Forschungseinrichtungen führen können. Für die Ansiedlung der neuen Abteilung des RKI „, Zentrum für Künstliche Intelligenz in der Public Health Forschung" in Wildau beispielsweise spielt die Nähe zu Forschungsthemen an der TH Wildau eine Rolle. Für den Forschungsbeschleuniger am DESY in Zeuthen deuten sich neue Anwendungen im Bereich der Krebstherapie an und damit entstehende Beziehungen zu Akteur/innen der Gesundheitsregion Lausitz.

Die in den Interviews sichtbar gewordenen technologischen Innovationsthemen für die Flughafenumfeldregion "Gesundheit“, „Digitalisierung“, „Mobilität/Verkehr“ wurden im Podium als Schwerpunkte bestätigt. Zusätzlich in die Diskussion gebracht wurden die Themen Klimaschutz und Energie. Aus dem Teilnehmerkreis kam der Vorschlag, die Ergebnisse der Analyse in die Arbeit des Dialogforums BER einzubinden. 
Im Anschluss an die Podiumsdiskussion wurde ein Voting unter Nutzung des Tools Mentimeter zu sieben Thesen durchgeführt, dessen Ergebnisse abschließend dargestellt sind. Mit der Abstimmung über die Thesen sollten weitere Handlungsansätze identifiziert bzw. die vorgeschlagenen Handlungsansätze präzisiert werden.

Mit deutlicher Mehrheit stimmten die Teilnehmer/innen dafür, dass die Flughafenumfeldregion derzeit noch kein von außen wahrnehmbares Profil hat (vgl. Darstellung 23).

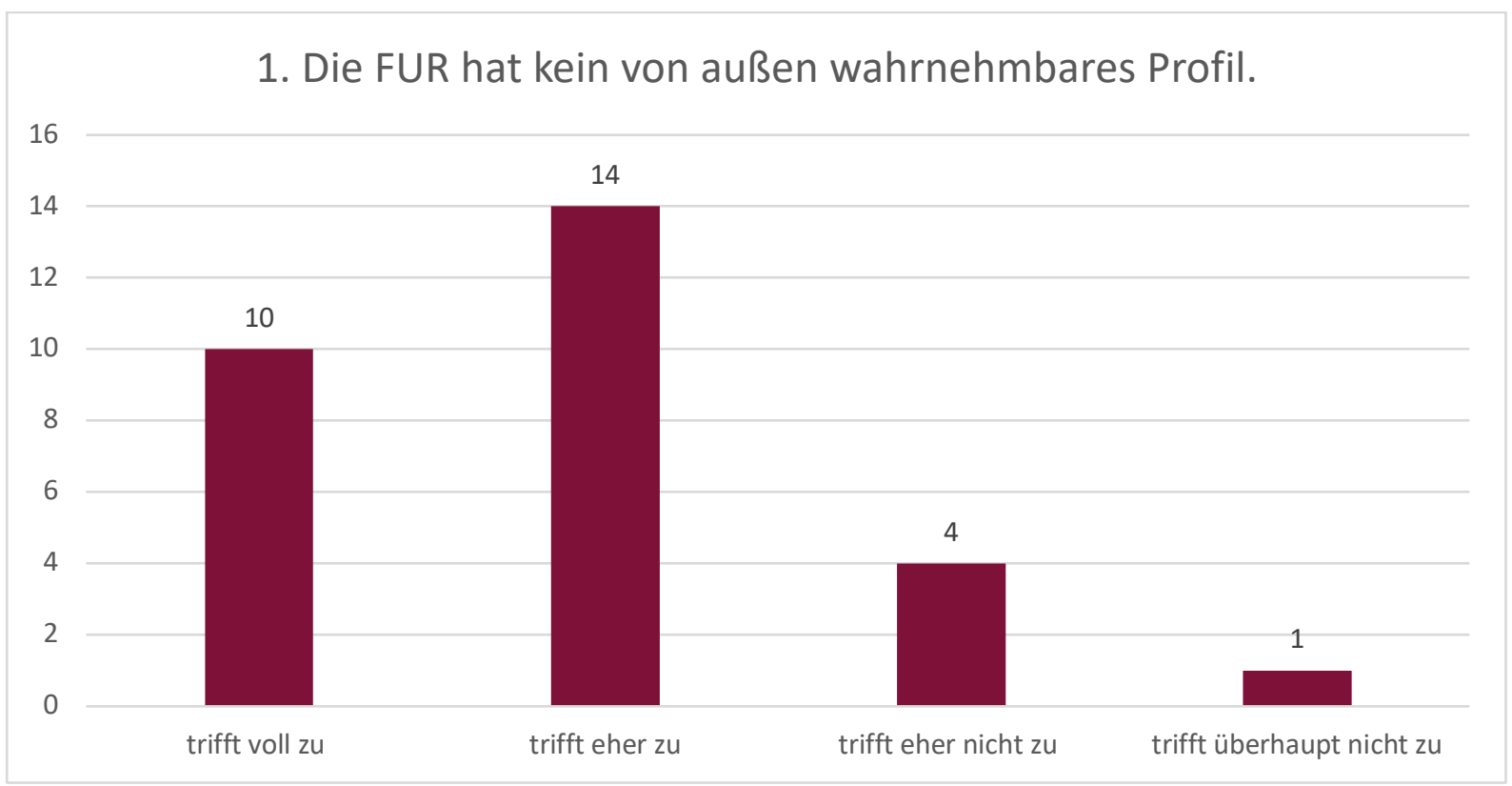

Darstellung 23: Abstimmungsergebnis zu These 1

Zu berücksichtigen ist hierbei, dass sich Selbstwahrnehmung und Fremdwahrnehmung durchaus unterscheiden können. Ein Vertreter des Podiums mit vielen internationalen Erfahrungen verwies darauf, dass die Region im Ausland sehr wohl als hoch dynamische Region wahrgenommen werde, auch und gerade im Zusammenhang mit der Ansiedlung von Tesla in Grünheide. Dennoch sind nach Einschätzung der Autor/innen gemeinsame Aktivitäten der Regionalakteur/innen bezüglich des Regionalmarketings, und zwar eines sowohl nach außen als auch nach innen gerichteten Regionalmarketings, erforderlich.

Bereits in den Interviews hatte sich angedeutet, dass die Flughafenumfeldregion dynamisch ist und sich entlang von Entwicklungsachsen in das Land Brandenburg hinein bzw. entlang des Autobahnringes profiliert. Diese These wurde im Voting von 24 Teilnehmer/innen bestätigt (vgl. Darstellung 24). 


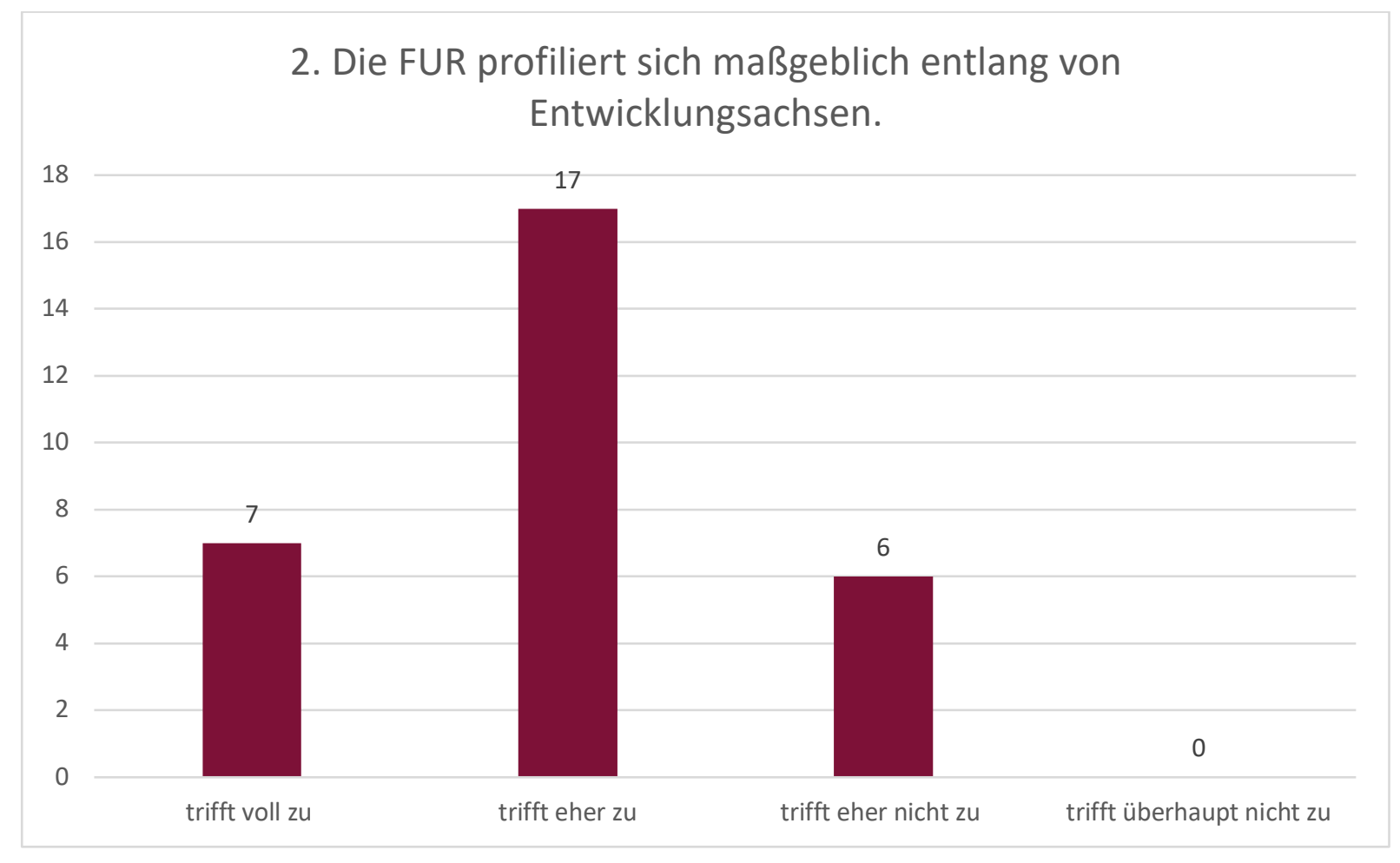

Darstellung 24: Abstimmungsergebnis zu These 2

Nicht so deutlich bestätigt wurde die These, dass die Regionalen Wachstumskerne Luckenwalde und Fürstenwalde sowie die Kreisstadt Lübben, die an den Rändern der Region liegen, Zukunftsorte der Flughafenumfeldregion seien. Die Teilnehmer/innen waren diesbezüglich geteilter Meinung (vgl. Darstellung 25$)$.

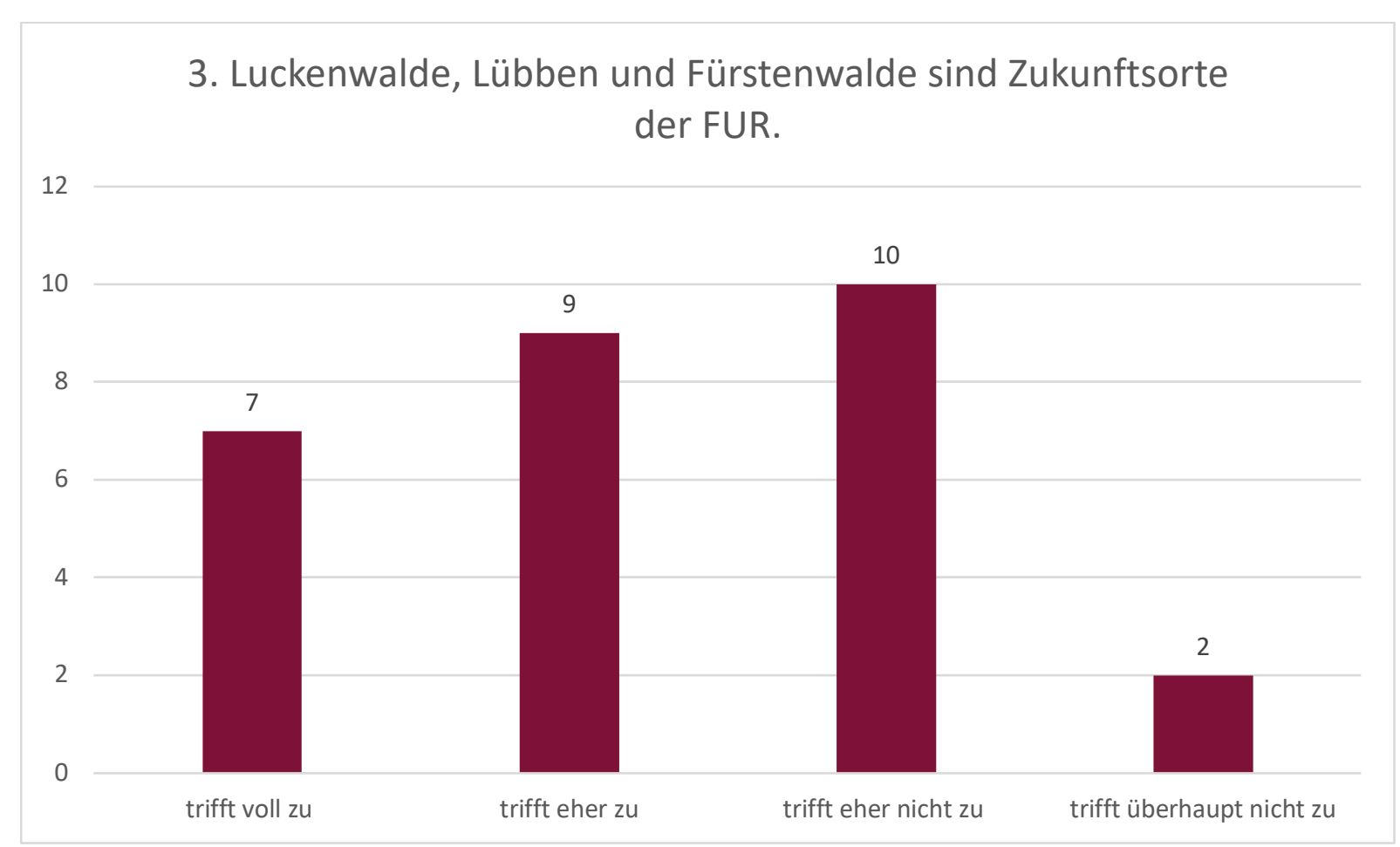

Darstellung 25: Abstimmungsergebnis zu These 3 
Ein wiederum sehr eindeutiges Abstimmungsergebnis ergab sich bezüglich der These zum Einbezug der Bevölkerung in die Gestaltung der Zukunft der Region. Fast alle Teilnehmer/innen halten diese für unzureichend (vgl. Darstellung 26), was die Umsetzung entsprechender Maßnahmen nahelegt. In den Handlungsansätzen wurde hierauf bereits Bezug genommen.

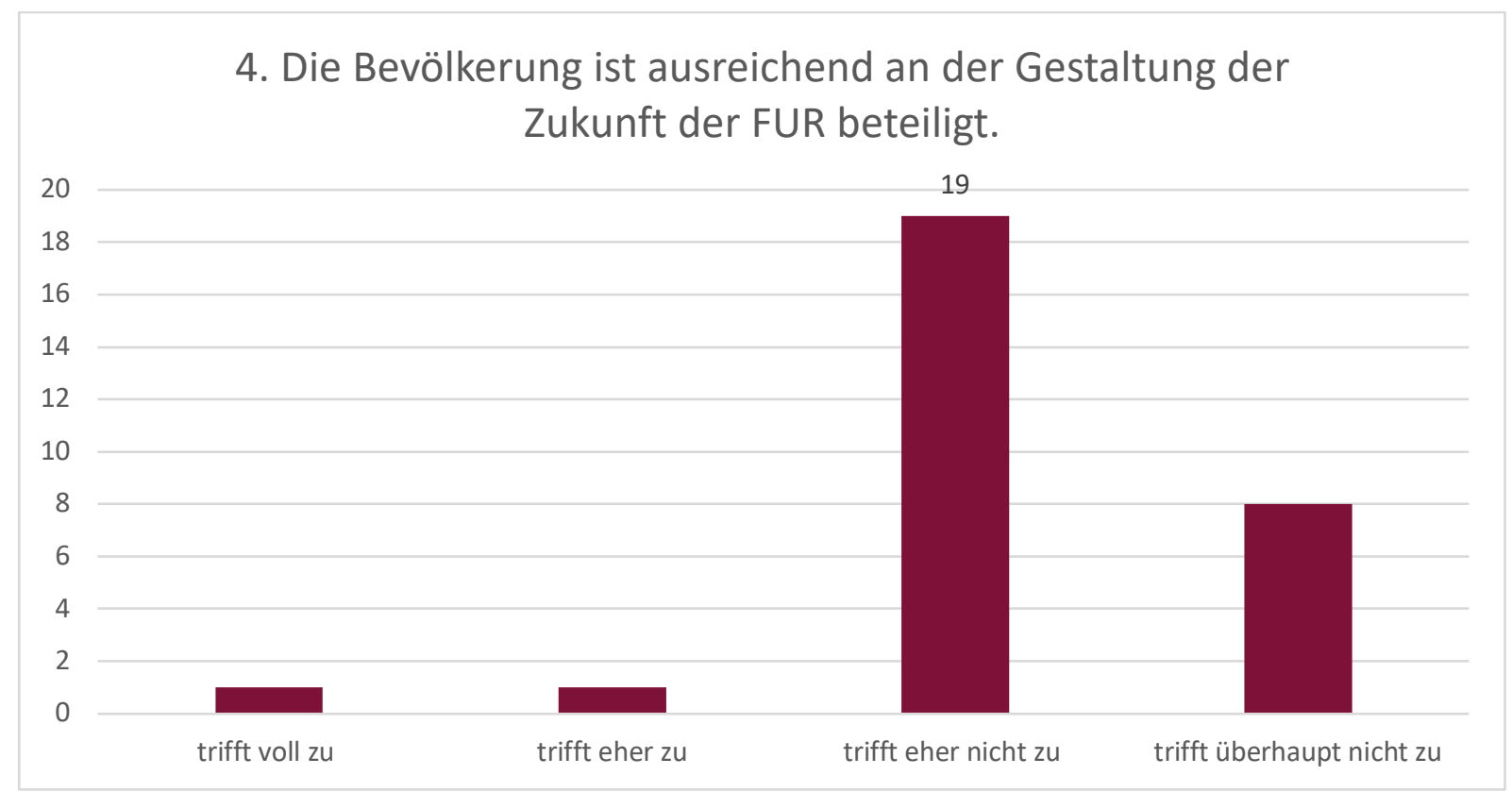

Darstellung 26: Abstimmungsergebnis zu These 4

These 5 zielte darauf ab, zu erkunden, ob die Teilnehmer/innen auch den Brandenburger Teil der Flughafenregion zukünftig als einen Wissenschafts- und Technologiestandort von solcher Bedeutung sehen, dass er auch international wahrgenommen wird. Offensichtlich ist das der Fall (vgl. Darstellung 27), wobei in der Diskussion zum Abstimmungsergebnis darauf hingewiesen wurde, dass die Region bereits heute ein international wahrgenommener Standort sei und die These darauf hindeutet, dass das vorhandene Potenzial unterschätzt wird. Im Rahmen der vorliegenden Analyse war eine vergleichende Darstellung der Wissenschafts- und Technologiepotenziale, etwa mit anderen Flughafenregionen, leider nicht möglich, so dass gut belegte Aussagen hierzu nicht getroffen werden können. 


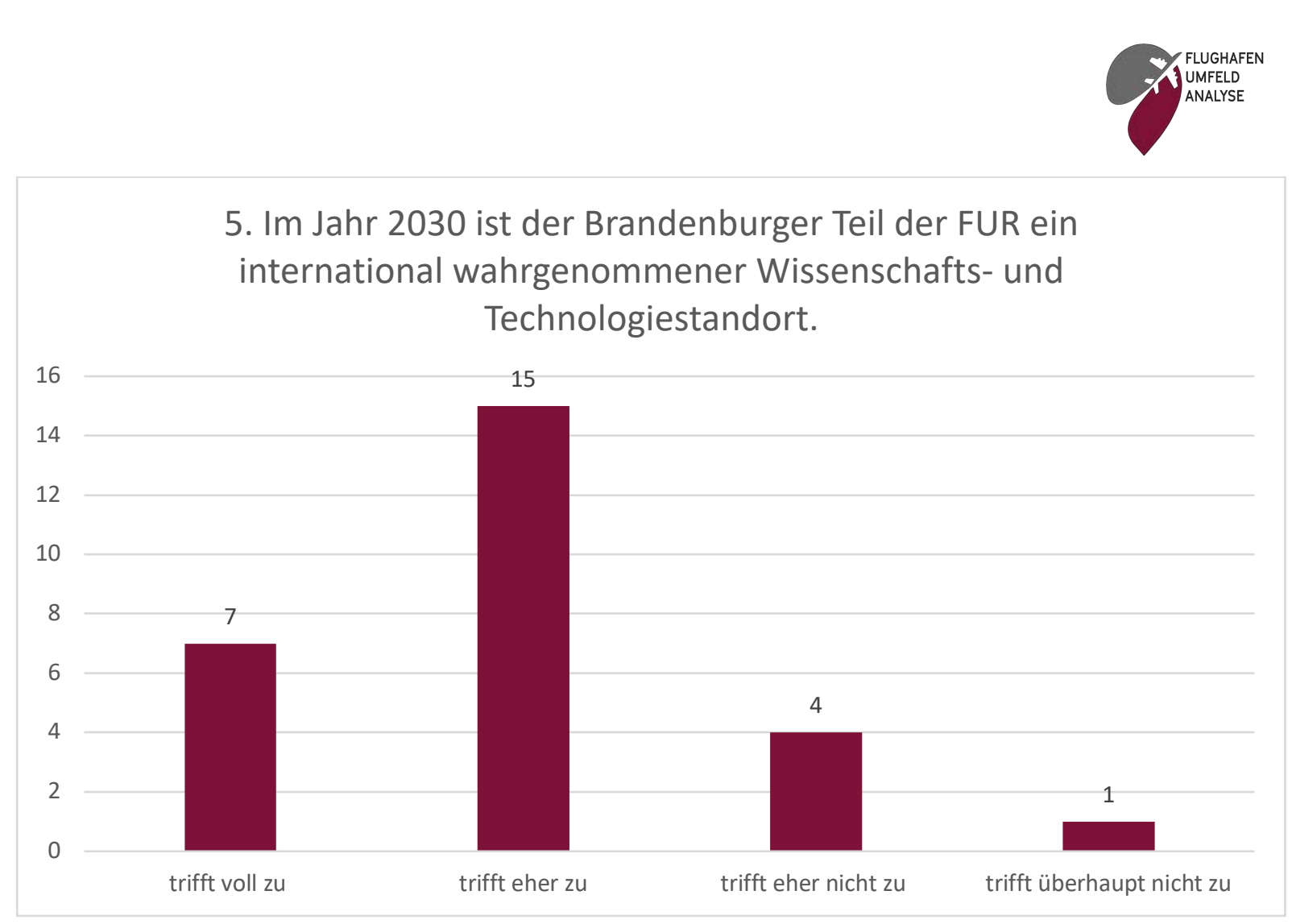

Darstellung 27: Abstimmungsergebnis zu These 5

Die Rolle von Startups in Flughafenumfeldregionen sollte mit der These sechs in das Interesse der verschiedenen Regionalakteur/innen gerückt werden. Gibt es Ansprüche von Startups, denen in einer Flughafenumfeldregion besonders gut entsprochen werden kann? Welche Rolle spielt die Nähe zu Wissenschaftseinrichtungen und anderen, bereits existierenden, technologieorientierten Unternehmen? Eine deutliche Mehrheit der Teilnehmer/innen sieht die Region als zukünftigen Hotspot für Startups. Auch bezogen auf dieses Thema findet sich weiter oben eine Handlungsansatz, der hiermit unterstrichen wird. 


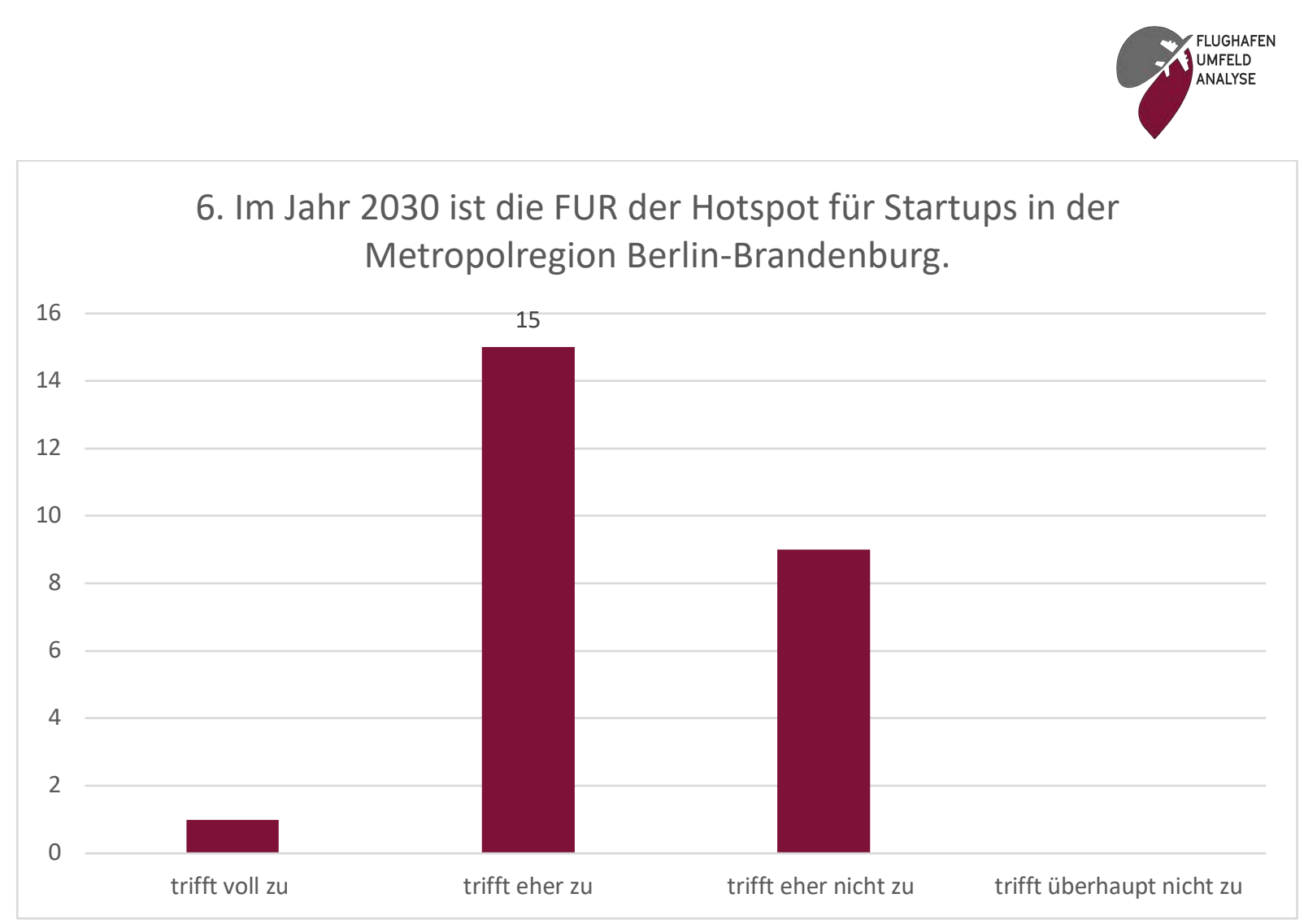

Darstellung 28: Abstimmungsergebnis zu These 6

Insbesondere das Good Practice Beispiel des Flughafens Münchens mit seinem Lab Campus führte zu der Frage nach einer direkteren Verbindung zwischen dem Flughafen und der Wissenschaft. Vorstellbar ist, dass er als Testbed für Innovationen und als Schaufenster für Wissenschaft der Metropolregion fungiert. Allerdings sahen nur 11 Teilnehmer/innen den Flughafen BER als ein solches Schaufenster für Wissenschaft und Technologie, 18 Personen eher nicht (vgl. Darstellung 29).

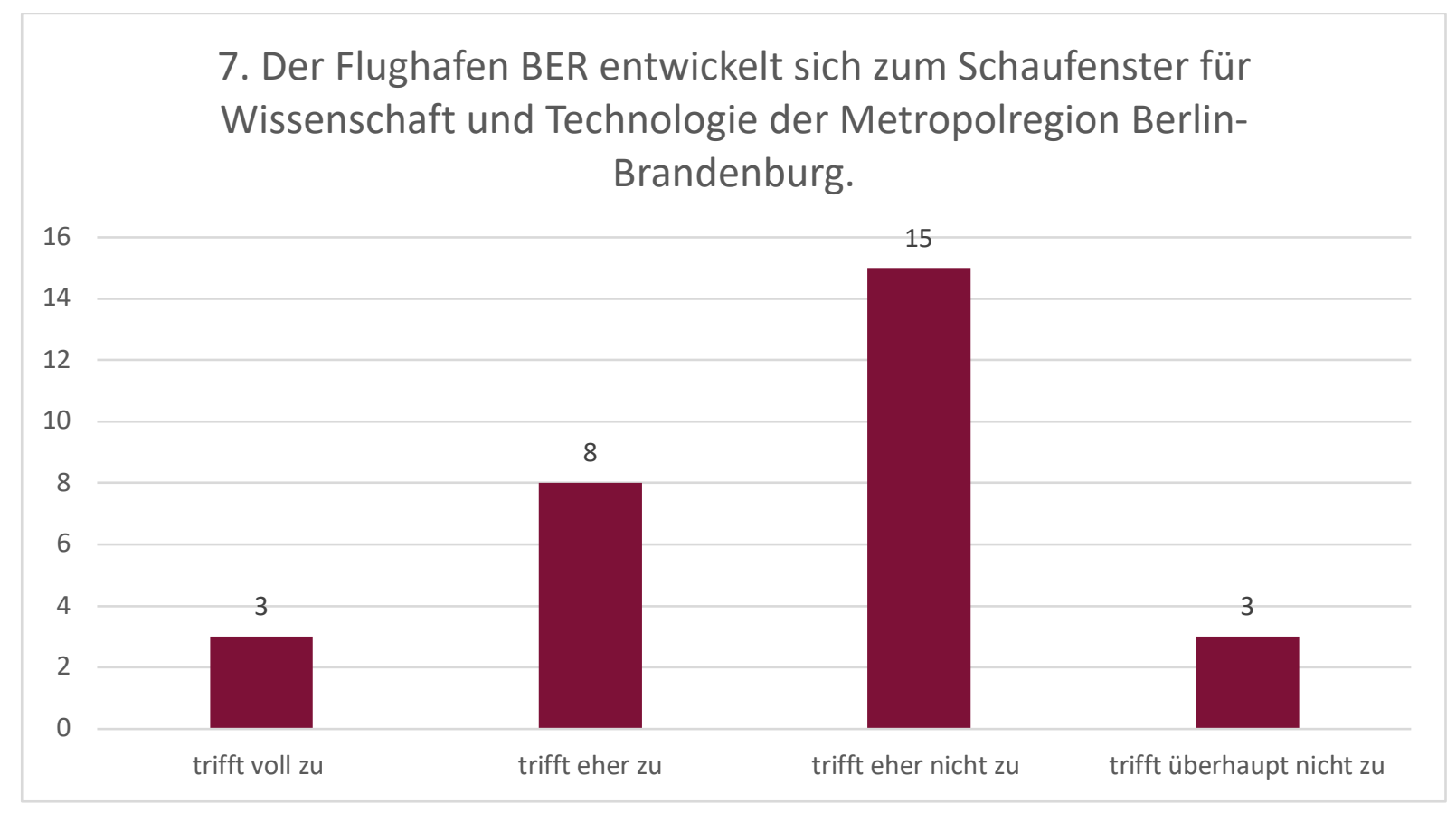

Darstellung 29: Abstimmungsergebnis zu These 7 
Insgesamt betrachtet hat das digitale Forum die Ergebnisse der Analyse bestätigt und die herausgearbeiteten Handlungsansätze unterstützt. Die nächsten Schritte beinhaltend die Aufnahme von Kooperationsbeziehungen der TH Wildau mit dem Dialogforum BER hinsichtlich der Entwicklung einer gemeinsamen Vision für die Flughafenumfeldregion sowie mit dem neuen Regionalmanagement bezüglich der Rolle von Wissenschaft und Technologie. Im Rahmen des Projektes Innovation Hub 13 - fast track to transfer, in dem sich ein neues Teilvorhaben Bürgerwissenschaft profiliert, lädt die TH Wildau alle interessierten Akteur/innen zur Zusammenarbeit ein, um eine stärkere Einbindung der Bürger/innen in die Entwicklung der Region zu erreichen. Gemeinsam mit der WFBB und in der Region aktiven Immobilienentwickler/innen wird eine stärkere Zusammenarbeit hinsichtlich der Ansiedlung von Wissenschaftseinrichtungen und technologieorientierten Unternehmen angestrebt. 


\section{Literaturverzeichnis}

Ache, P. (2000). Was treibt den Motor an? Stadtregionen zwischen innovativen Milieus und Urban Governance. Raumforschung und Raumordnung 58(2-3), 244-253.

Adlershof a). (2020). Photonik/Optik. [Online]. Verfügbar unter: https://www.adlershof.de/fileadmin/user_upload/downloads/technologie/Zentrum_fuer_Photonik_und_Optik.pdf. [letzter Zugriff: 15.06.2020].

Adlershof b). (2020). Mikrosystem/Materialien. [Online]. Verfügbar unter: https://www.adlershof.de/wirtschaft-wissenschaft/technologiezentren/mikrosysteme-materialien/info/. [letzter Zugriff: 15.06.2020].

Adlershof c). (2020). IT/Medien. [Online]. Verfügbar unter: https://www.adlershof.de/wirtschaft-wissenschaft/technologiezentren/it-medien/info/. [letzter Zugriff: 15.06.2020].

Adlershof d). (2020). Biotechnologie/Umwelt. [Online]. Verfügbar unter: https://www.adlershof.de/wirtschaft-wissenschaft/technologiezentren/biotechnologie-umwelt/info/. [letzter Zugriff: 15.06.2020].

Adlershof e). (2020). Erneuerbare Energien/Photovoltaik. [Online]. Verfügbar unter: https://www.adlershof.de/fileadmin/user_upload/downloads/technologie/Zentrum_fuer_Photovoltaik_und_erneubare_Energien.pdf. [letzter Zugriff: 15.06.2020].

Adlershof f). (2020). Außeruniversitäre Forschungseinrichtungen und Campus Humboldt-Universität. [Online]. Verfügbar unter: https://www.adlershof.de/wirtschaft-wissenschaft/ausseruniversitaereforschung/ueberblick/. [letzter Zugriff: 15.06.2020] sowie https://www.adlershof.de/wirtschaft-wissenschaft/humboldt-universitaet-zu-berlin/ueberblick/. [letzter Zugriff: 15.06.2020].

Arbeitsagentur. (2020). Stellensuche. [Online]. Verfügbar unter: www.con.arbeitsagentur.de/prod/jobboerse. [letzter Zugriff 27.02.2020].

Bittelmeyer, C., Ehrhart, N. und Zimmermann, V. (2007). Einflussfaktoren auf das Patentierungsverhalten von kleinen und mittleren Hightech-Unternehmen des verarbeitenden Gewerbes. Mittelstands- und Strukturpolitik. Frankfurt, M. KfW-Bankgruppe. Vol. 39.2007, S. 43-80.

Cooke, P. (2001). Regional Innovation Systems, Clusters, and the Knowledge Economy. Industrial and corporate change, 10, 945-974.

Deutsches Patent- und Markenamt (o. J.). Patente 2019. [Online]. Verfügbar unter: https://www.dpma.de/docs/presse/dpma_infografik_patente_dt_2019.pdf. [letzter Zugriff: 02.09.2020].

Dialogforum-BER. (2020). Kurzinformation GSK-Fortschreibung Juli 2020. [Online]. Verfügbar unter: https://www.dialogforum-ber.de. [letzter Zugriff: 18.11.2020].

Fischer, O. (2017). Rolls-Royce ist Innovationsmotor der Region. MAZ vom 28.07.2017. [Online]. Verfügbar unter: https://www.maz-online.de/Lokales/Teltow-Flaeming/Rolls-Royce-ist-Innovationsmotor-der-Region. [letzter Zugriff: 17.08.2020].

Fischer, O. (2019). Wachstum hält unvermindert an. [Online]. Verfügbar unter: https://www.maz-online.de/Lokales/Teltow-Flaeming/Dahme-Spreewald-und-Teltow-Flaeming-Wachstum-haelt-unvermindert-an. [letzter Zugriff: 24.02.2020].

Fürstenwalde-Spree, (o.J.). Regionaler Wachstumskern Fürstenwalde-Spree. [Online]. Verfügbar unter: https://www.fuerstenwalde-spree.de/seite/162129/rwk.html. [letzter Zugriff: 14.12.2020]. 
Gruschwitz, S. (2018). Strukturkonzept. [Online]. Verfügbar unter: https://www.luckenwalde.de/Wirtschaft/Standortentwicklungskonzept. [letzter Zugriff: 24.02.2020].

GSK. (2007). Gemeinsames Strukturkonzept Flughafenumfeld Berlin Brandenburg International (BBI). Hrsg.: Ministerium für Infrastruktur und Raumordnung und Senatsverwaltung für Stadtentwicklung.

Harhoff, D. (2010). Patente in mittelständischen Unternehmen. Eine empirische Studie des Instituts für Innovationsforschung, Technologiemanagement und Entrepreneurship. [Online]. Verfügbar unter: https://epub.ub.uni-muenchen.de/13119/1/Harhoff_1547.pdf. [letzter Zugriff: 03.09.2020].

Henn, S. und Werner, P. (2016). Erfindungsaktivitäten in Deutschland - Patente, Gebrauchsmuster, Marken und Design. In: Nationalatlas aktuell 10 (11.2016) 9 [05.12.2016]. Leipzig: Leibniz-Institut für Länderkunde (IfL). [Online]. Verfügbar unter: http://aktuell.nationalatlas.de/Patente.9_122016.0.html. [letzter Zugriff: 03.09.2020].

Immobilienscout24. (2020). Anzahl Mietwohnung und Häuser zum Verkauf. [Online]. Verfügbar unter: https://www.immobilienscout24.de/. [letzter Zugriff: 28.03.2020].

Innovationspark Wuhlheide. (2020). [Online]. Verfügbar unter: https://ipw-berlin.info/wir-ueberuns/unternehmen-im-ipw/. [letzter Zugriff: 15.06.2020].

LABCampus. (2020). [Online]. Verfügbar unter: https://www.labcampus.de/. [letzter Zugriff: 17.11.2020]

Ludwigsfelde. (o. J.). US-Batteriehersteller verlegt Europasitz nach Ludwigsfelde. [Online]. Verfügbar unter: https://www.ludwigsfelde.de/us-batteriehersteller-verlegt-europasitz-nach-ludwigsfelde/. [letzter Zugriff: 24.11.2020].

IAU. (2018). Sustainable Airport Areas. Guidelines for Decision Makers. [Online]. Verfügbar unter: https://www.metropolis.org/sites/default/files/resources/2018.03_Sustainable_Airport_Areas_Guidelines_for_decision_makers_0.pdf. [letzter Zugriff: 23.11.2020].

Meyer, S. (2020). Tesla in Grünheide. LR Online. [Online]. Verfügbar unter: https://www.Ir-online.de/nachrichten/brandenburg/tesla-in-gruenheide-das-sind-die-neuen-jobs-in-elon-musks-gigafactory-43845735.html. [letzter Zugriff: 23.11.2020].

MTU. (2020). MTU Aero Engines plant Anpassung der Personalkapazität um 10 bis 15 Prozent bis Ende 2021. [Online]. Verfügbar unter: https://www.mtu.de/de/newsroom/presse/aktuelle-presseinformationen/press-release-detail/mtu-aero-engines-plans-to-adjust-personnel-capacity-by-10-to-15percent-by-the-end-of-2021/. [letzter Zugriff: 24.11.2020].

Musil, R und Eder, J. (2017). Wozu räumliche Nähe in der urbanen Wissensökonomie? Eine geostatistische Analyse Wiener Forschungscluster. Raumforschung und Raumbeobachtung 2017, 75:93-107.

PatBase. (o. J.). What is PatBase? [Online]. Verfügbar unter: https://www.patbase.com/. [letzter Zugriff: 17.08.2020].

Porter, M. E. (1998). Clusters and the new economics of competition. Harvard Business Review. November - December 1998. [Online]. Verfügbar unter: https://hbr.org/1998/11/clusters-and-the-neweconomics-of-competition. [letzter Zugriff: 14.10.2019].

rbb. (2020). Stellenabbau beim Autozulieferer. Schaeffler in der Krise - Werk in Luckenwalde könnte verkauft werden. [Online]. Verfügbar unter: https://www.rbb24.de/wirtschaft/bei-

trag/2020/09/brandenburg-schaeffler-baut-tausende-stellen-ab-luckenwalde.html. [letzter Zugriff: 24.11.2020].

Schibany, A. und Dachs, B. (2003). Patente. Ein Indikator für technologische Leistungsfähigkeit und Internationalisierung. WIFI Studies, WIFO, number 24788, December. 
statistik Berlin Brandenburg. (2020). [Online]. Verfügbar unter: https://www.statistik-berlin-brandenburg.de/webapi/jsf/tableView/tableView.xhtm. [letzter Zugriff: 24.04.2020].

Technologie- und Gründerzentrum Spreeknie. (2020). [Online]. Verfügbar unter: https://tgs.berlin/wir-ueber-uns/unternehmen-im-tgs/. [letzter Zugriff: 15.06.2020].

SPV. (2019). Grundlagenermittlung Verkehr Flughafenregion BER. Abschlussbericht August 2019. Spreeplan Verkehr GmbH. [Online]. Verfügbar unter: https://daten2.verwaltungsportal.de/dateien/seitengenerator/8bb9f9a3f80aa77b39a2287cc1545b2c194355/2019_08_grundlagenermittlung_verkehr_-_kag_ber_bericht_endfassung_1.pdf. [letzter Zugriff: 30.11.2020].

Tesla. (o. J.). Gigafactory Berlin-Brandenburg. [Online]. Verfügbar unter: https://www.tesla.com/de_de/gigafactory-berlin. [letzter Zugriff: 24.11.2020].

Wagner, D. und Schultz, C. (2011). Finanzierung technologieorientierter Unternehmen in Deutschland. EUL Verlag. Köln.

Wangemann, U. und Gellner, T. (2020). MAZ. Triebwerkshersteller Rolls-Royce baut 550 Stellen in Brandenburg ab. [Online]. Verfügbar unter: https://www.maz-online.de/Brandenburg/DahlewitzTriebwerkshersteller-Rolls-Royce-baut-550-Stellen-ab. [letzter Zugriff: 24.11.2020].

WFBB. (o. J.). Standort Brandenburg. Branchen und Cluster. [Online]. Verfügbar unter: https://www.wfbb.de/de/Wirtschaftsstandort/Branchen-und-Cluster/. [letzter Zugriff: 13.05.2020].

Wohnungsbörse. (2020). Mietspiegel Berlin 2020. [Online]. Verfügbar unter: https://www.wohnungsboerse.net/mietspiegel-Berlin/2825. [letzter Zugriff: 26.11.2020].

ZEW. (2019). Innovationen in der deutschen Wirtschaft. Indikatorenbericht zur Innovationserhebung 2018. [Online]. Mannheim. Verfügbar unter: http://ftp.zew.de/pub/zew-docs/mip/18/mip_2018.pdf. [letzter Zugriff: 05.12.2020]. 
Anlagen 


\section{Anlage 1 Gemeindesteckbriefe}

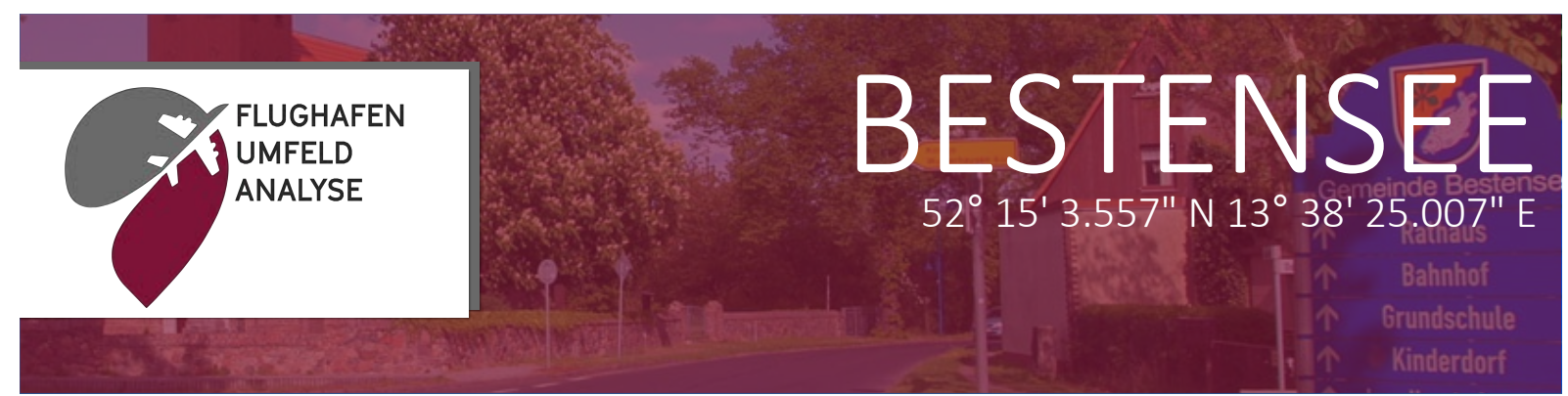

Lage und Besonderheiten
\& Einwohner/innen
7.850 (Stand: März 2020)

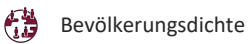
$206 \mathrm{EW} / \mathrm{km}^{2}$
Hö Fläche
$38,04 \mathrm{~km}^{2}$
A Infrastruktur
direkter Autobahnanschluss an A13; direkter Anschluss an Regionalverkehr der DB, BER $23 \mathrm{~km}$ entfernt
$\varkappa^{\star} \quad$ Leitbild der Gemeinde $\quad$ k.A

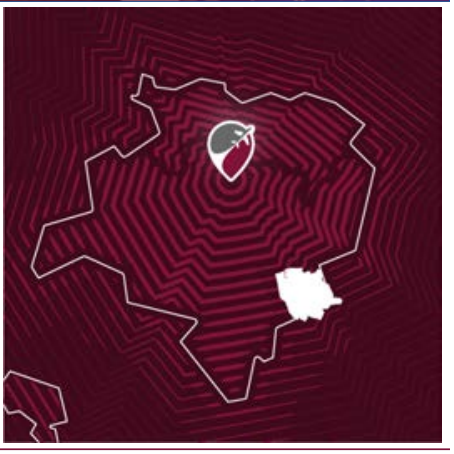

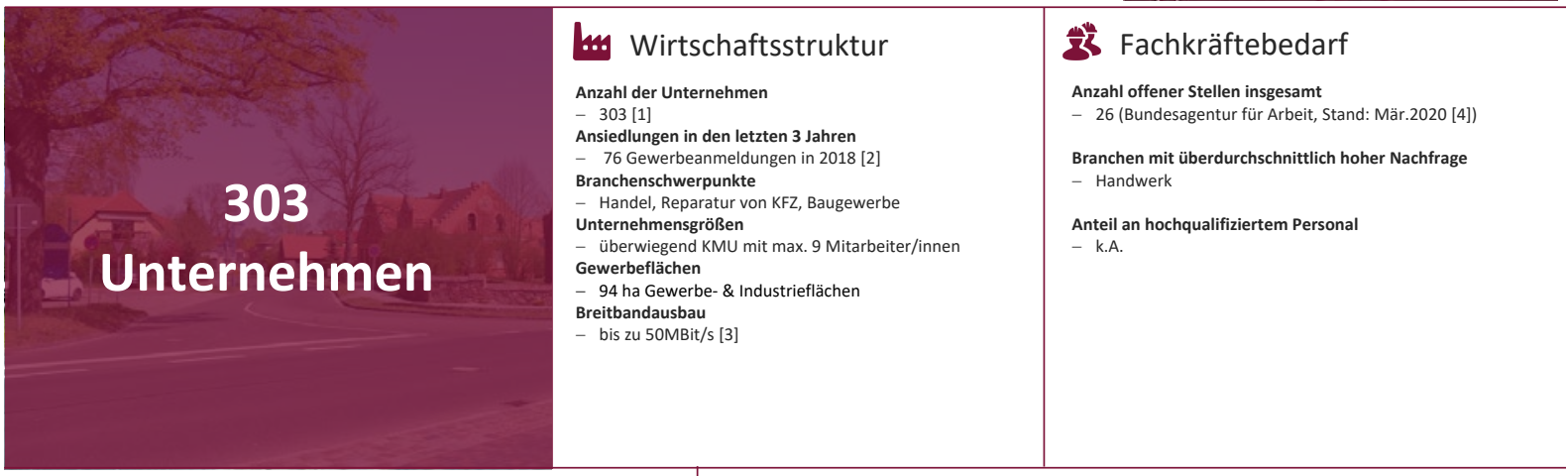

Wissenschaft und Forschung Anzahl Forschungseinrichtungen - k.A.

Art der Einrichtungen

- k.A.

Inhaltliche Schwerpunkte

- k.A.
洨: Innovationsstärke

Testbeds

k.A.

Spezifische Netzwerke zur Unterstützung der Innovationsfähigkeit von Unternehmen

k.A.

Öffentlich geförderte Projekte

k.A.

\begin{tabular}{|c|c|}
\hline $\begin{array}{l}\text { Leben und Wohnen } \\
\text { Mietpreisentwicklung } \\
- \text { aktuell: } 7,12 \text { EUR/ } \mathrm{m}^{2} / 2011: 5,03-8,50 \mathrm{EUR} / \mathrm{m}^{2} \text { (Stand: März } 2020 \text { [5]) } \\
\text { Verfügbarkeit von Wohnraum } \\
-4 \text { Mietwohnungen, } 79 \text { Häuser zum Kauf, } 6 \text { Baugrundstücke (Stand: März } 2020 \text { [6]) } \\
\text { Medizinische Versorgung } \\
-8 \text { niedergelassene Ärzte versch. Fachdisziplinen } \\
\text { Bildungsangebot } \\
-1 \text { Grundshule } \\
\text { Umweltqualität } \\
- \text { viele Seen und Wälder in der Umgebung } \\
\text { Kulturangebot } \\
- \text { kulturhistorischer Wanderpfad, Generationenwald }\end{array}$ & $\begin{array}{l}1 \text { USP - Das zeichnet die Gemeinde aus } \\
\text { - Idyllische Landschaft mit Seen und Wiesen } \\
\text { - viele Wandermöglichkeiten }\end{array}$ \\
\hline 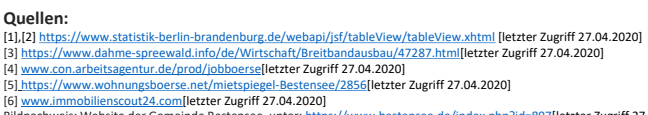 & \\
\hline
\end{tabular}




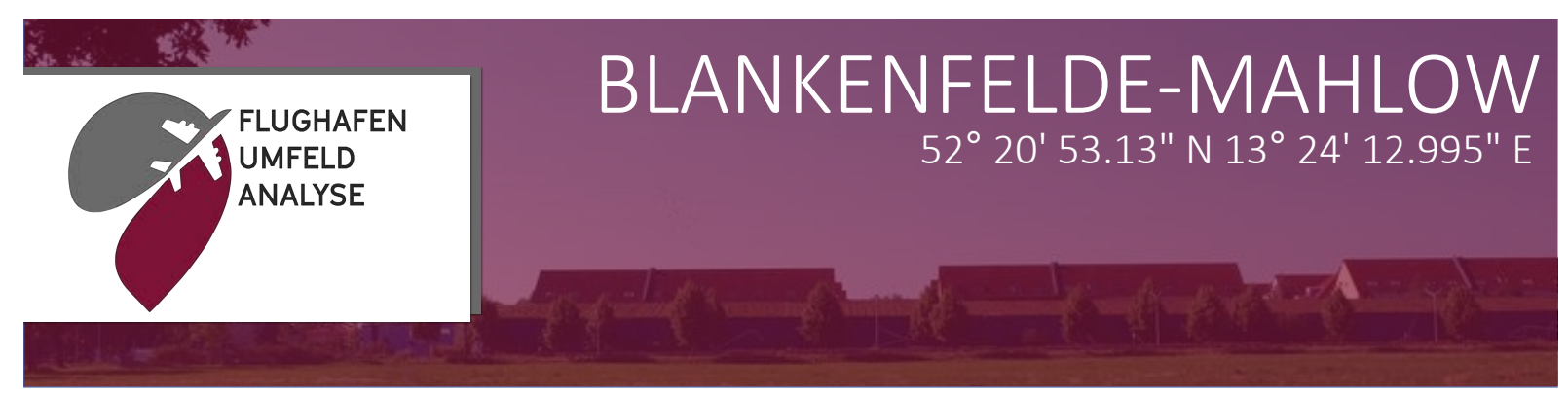

Lage und Besonderheiten

$\begin{array}{lll}\text { S3 Einwohner/innen } & 28.320 \text { (Stand: März 2020) } \\ & 516 \mathrm{EW} / \mathrm{km}^{2} \\ & 54,89 \mathrm{~km}^{2}\end{array}$
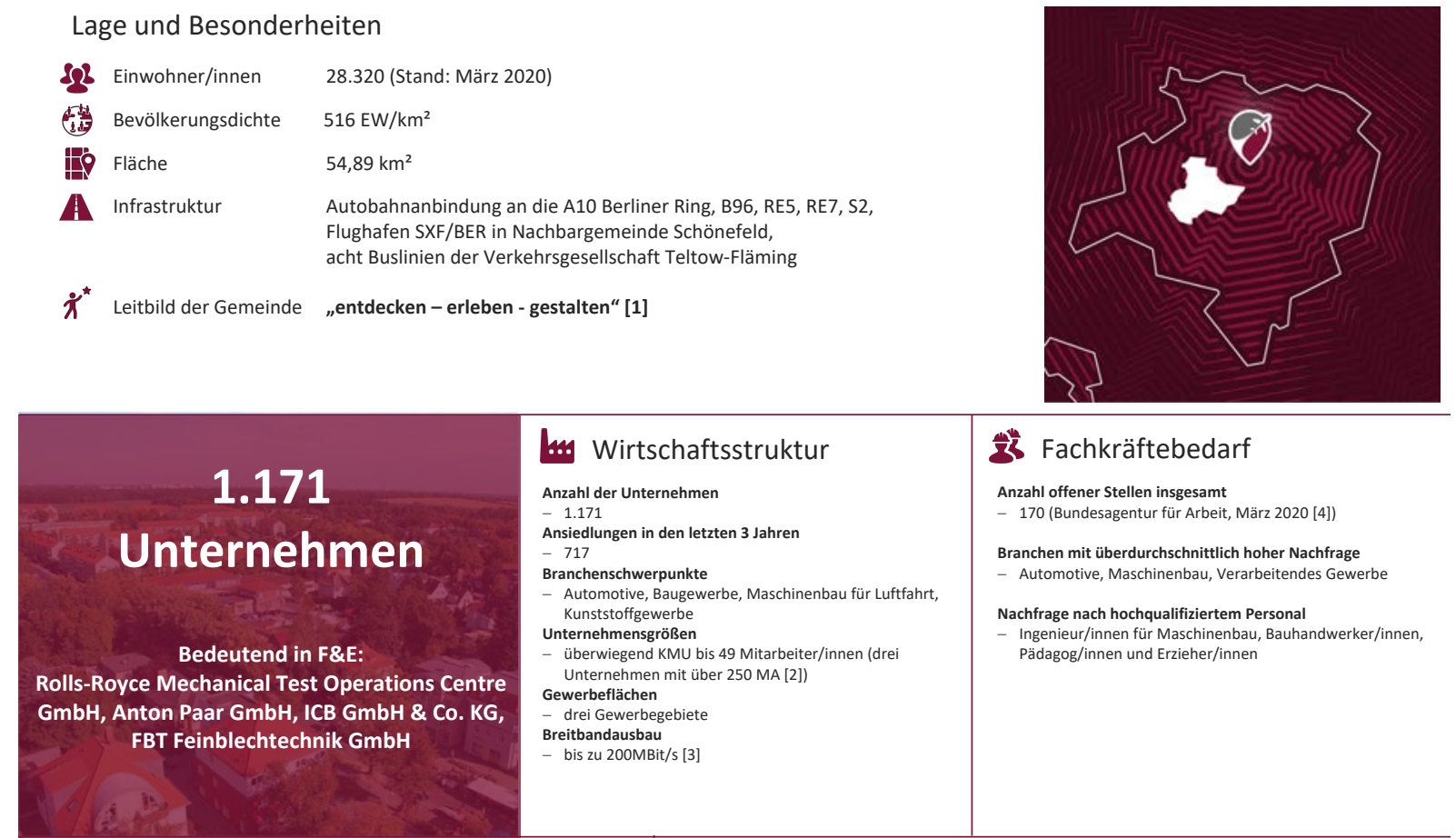

\begin{tabular}{|c|c|}
\hline Wirtschaftsstruktur & 胱 Fachkräftebedarf \\
\hline Anzahl der Unternehmen & Anzahl offener Stellen insgesamt \\
\hline $\begin{array}{l}-1.171 \\
\text { Ansiedlungen in den letzten } 3 \text { Jahren }\end{array}$ & - 170 (Bundesagentur für Arbeit, März 2020 [4]) \\
\hline $\begin{array}{l}\text { Ansiedlungen in den letzten } 3 \text { Jahren } \\
-717\end{array}$ & Branchen mit überdurchschnittlich hoher Nachfrage \\
\hline $\begin{array}{l}\text { Branchenschwerpunkte } \\
\text { - Automotive, Baugewerbe, Maschinenbau für Luftfahrt, }\end{array}$ & - Automotive, Maschinenbau, Verarbeitendes Gewerbe \\
\hline $\begin{array}{l}\text { Kunststoffgewerbe } \\
\text { Unternehmensgrößen } \\
\text { - überwiegend KMU bis } 49 \text { Mitarbeiter/innen (drei }\end{array}$ & $\begin{array}{l}\text { Nachfrage nach hochqualifiziertem Personal } \\
\text { - Ingenieur/innen für Maschinenbau, Bauhandwerker/innen, } \\
\text { Pädagog/innen und Erzieher/innen }\end{array}$ \\
\hline
\end{tabular}

Rolls-Royce Mechanical Test Operations Centre Unternehmen mit über 250 MA [2]) Gewerbeflächen

Breitbandausbau

- bis zu 200MBit/s [3]

Wissenschaft und Forschung Anzahl Forschungseinrichtungen - k.A.

Art der Einrichtungen

- k.A.

Inhaltliche Schwerpunkte

- k.A.

\section{:- Innovationsstärke}

\section{Testbeds}

- Institut für Strahlenschutz und Qualitätsmanagement

- XL-Testbed von Rolls Royce: "Big Data" im Einsatz - Engine Health Monitoring bei Geschäftsflugzeugen [5]

Spezifische Netzwerke zur Unterstützung der Innovationsfähigkeit von Unternehmen

- in Planung: „Innovations- und Forschungscluster Luftfahrt"

Öffentlich geförderte Projekte

K.A.

\begin{tabular}{|c|c|}
\hline $\begin{array}{l}\text { Leben und Wohnen } \\
\text { Mietpreisentwicklung } \\
\text { - aktuell: } 10,13 \mathrm{EUR} / \mathrm{m}^{2} / 2011: 6,74 \mathrm{EUR} / \mathrm{m}^{2} \text { für } 60 \mathrm{~m}^{2} \text { (Stand: März } 2020 \text { [6]) } \\
\text { Verfügbarkeit von Wohnraum } \\
-23 \text { Mietwohnungen, } 6 \text { Eigentumswohnungen, } 10 \text { Häuser zur Miete (97 zum Kauf), } 18 \text { Baugrundstücke } \\
\text { (Stand: März } 2020 \text { [7]) } \\
\text { Medizinische Versorgung } \\
\text { - } 27 \text { niedergelassene Allgemein- und Fachärzte, ein Gesundheitszentrum } \\
\text { Bildungsangebot } \\
\text { - vier Grundschulen, eine Oberschule, ein Gymnasium sowie zwei Schulen in freier Trägerschaft [1] } \\
\text { Umweltqualität } \\
\text { - ausgedehnte Felder und Grünzüge sowie der Rangsdorfer See im Süden der Gemeinde } \\
\text { - dichtes Netz aus Bächen, Wiesen- und Waldarealen im Gutspark Dahlewitz } \\
\text { Kulturangebot } \\
- \text { neun Kirchengemeinden, Schloss Jünsdorf, Wasserturm Dahlewitz, Bibliothek, Kulturzentrum „Alte Aula“ }{ }^{[8]}\end{array}$ & $\begin{array}{l}1 \text { USP - Das zeichnet die Gemeinde auS } \\
\text { - Sitz zahlreicher Großkonzerne (z. B. Roll-Royce) und gut ausgelastete } \\
\text { Gewerbegebiete } \\
\text { - Gewerbeflächenpotential in zwei der drei Gewerbegebiete } \\
\text { - betont generationsübergreifendes Zusammenleben in organisch } \\
\text { gewachsener Gemeindestruktur } \\
\text { - Dachmarke „Groß Grün“ zur Förderung der Region, ihrer Vielfalt und } \\
\text { der Gemeinschaft } \\
\text { - Mischung aus ländlicher Beschaulichkeit und kleinstädtischem Flair } \\
\text { - historisch wertvolle Bau- und Kunstschätze [1], [3] }\end{array}$ \\
\hline 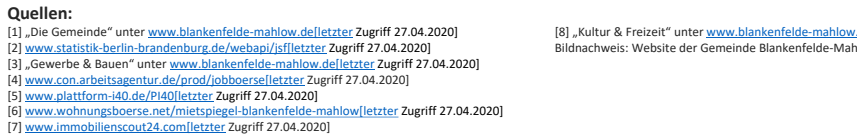 & $\begin{array}{l}\text { [8], Kultur \& Freizeit" unter www. blankenfelde-mahlow.de [letzter Zugriff 27.04.2020] } \\
\text { Bildnachweis: Website der Gemeinde Blankenfelde-Mahlow, unter httrps://www.blankenfelde-mahlow.de/Die-Gemeindelletzter [letzter Zugriff 27.04.2020] }\end{array}$ \\
\hline
\end{tabular}




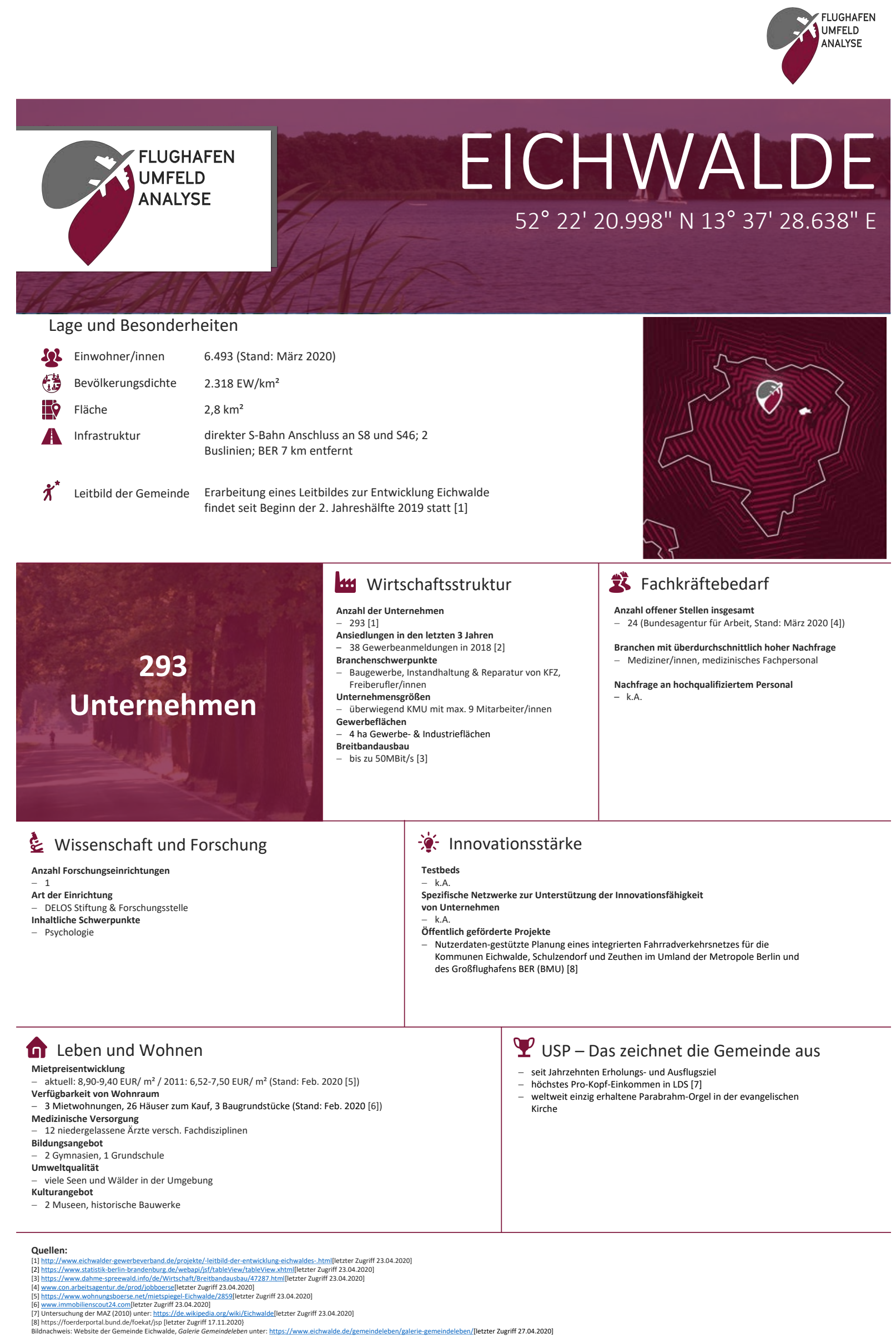




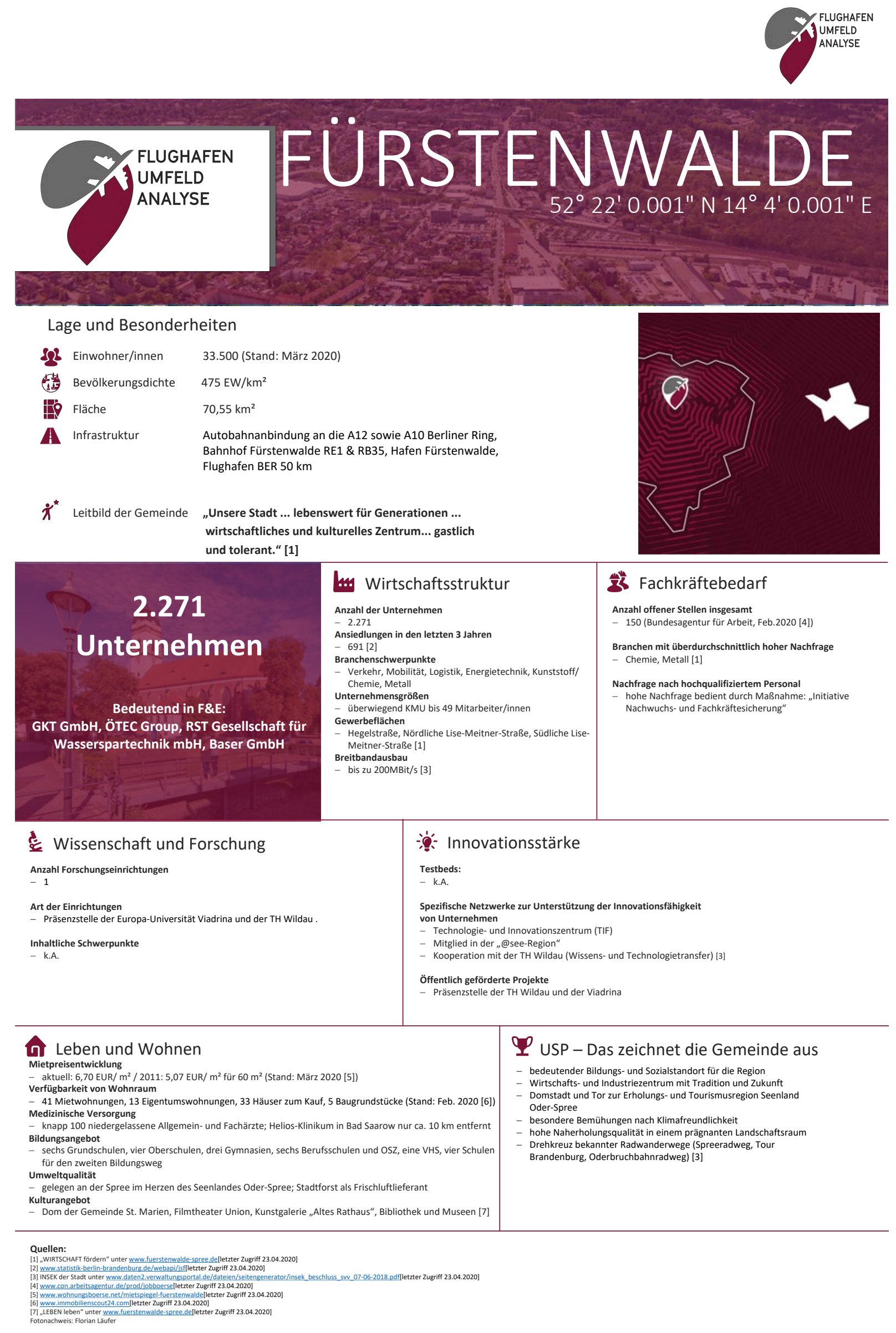




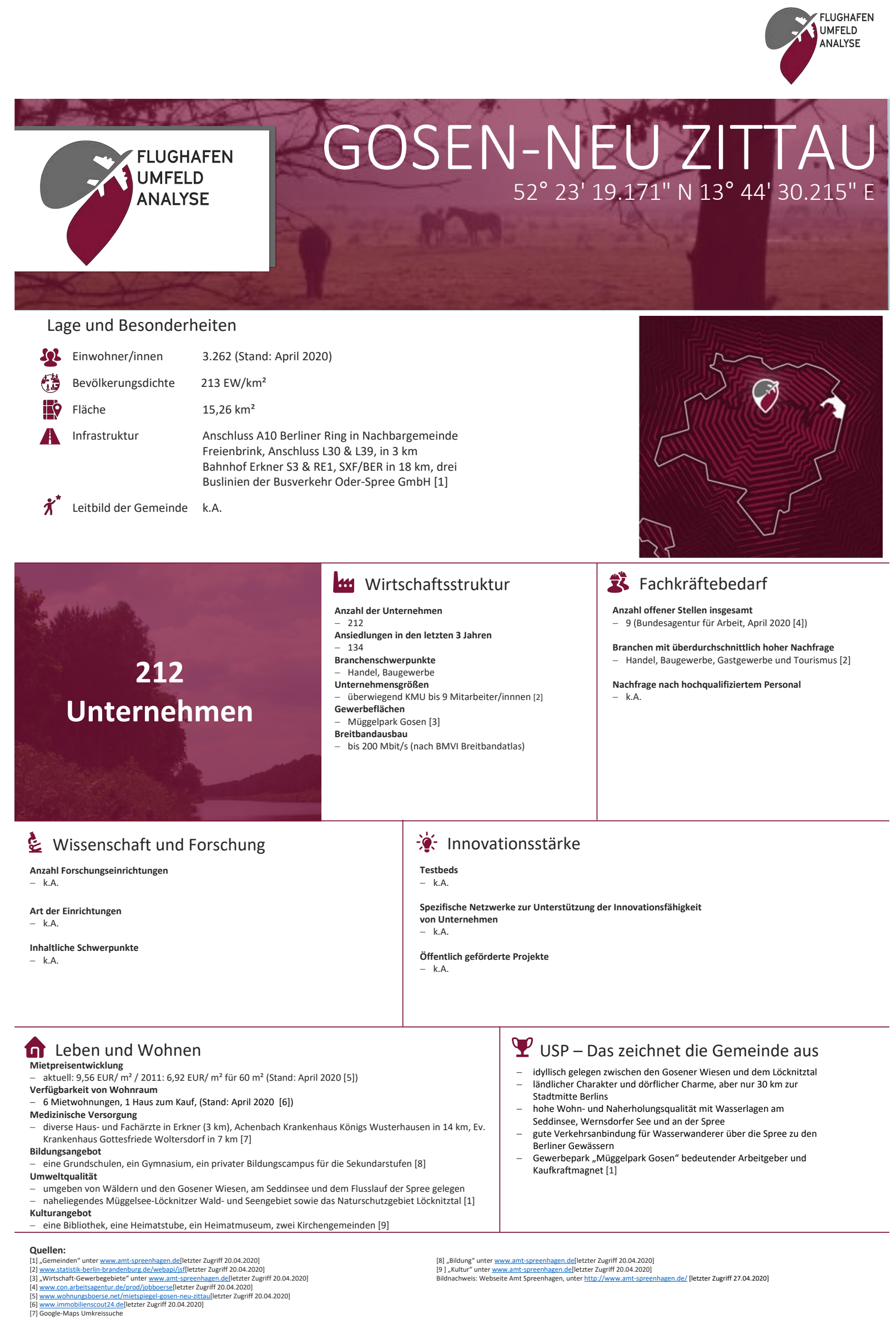




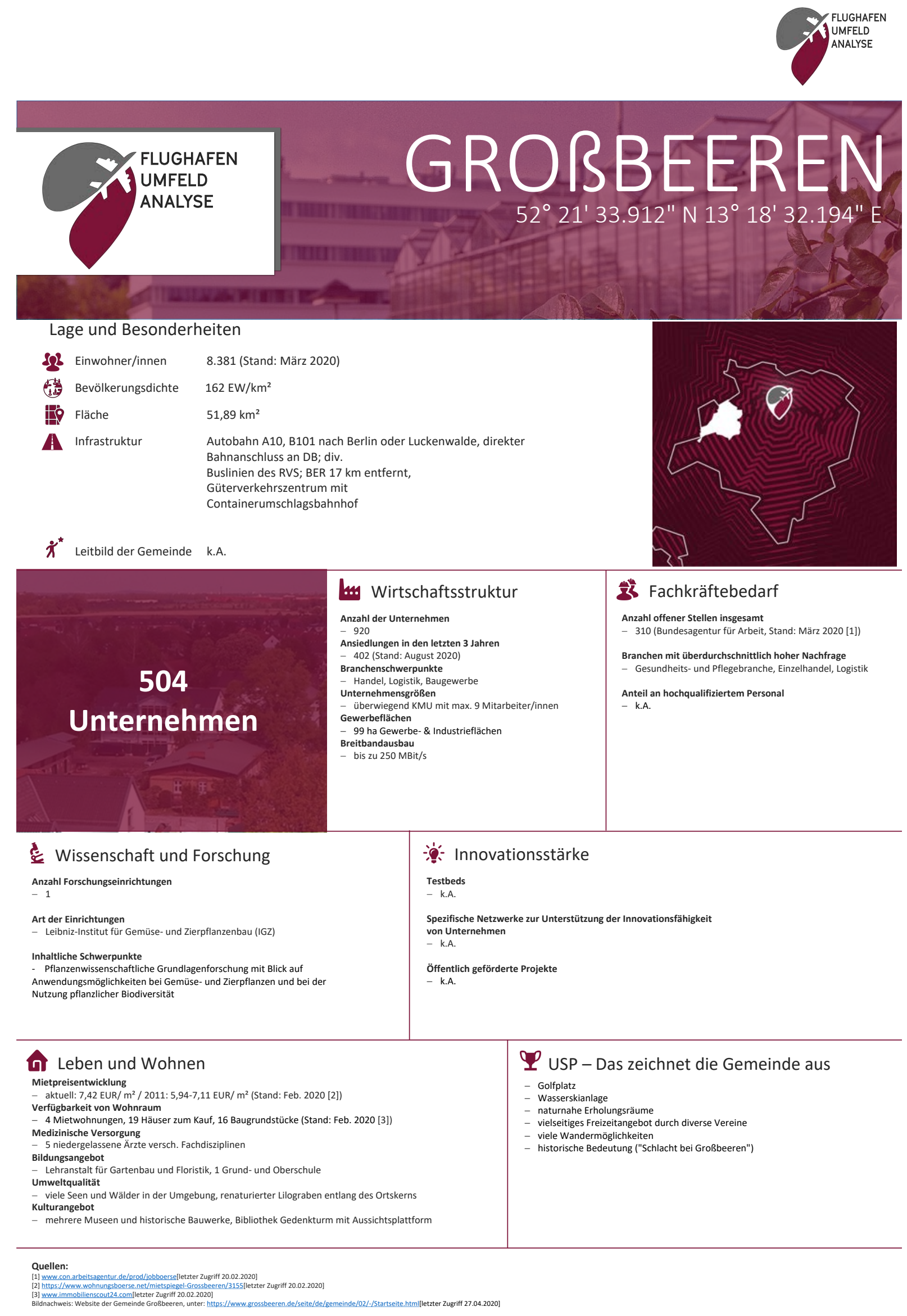




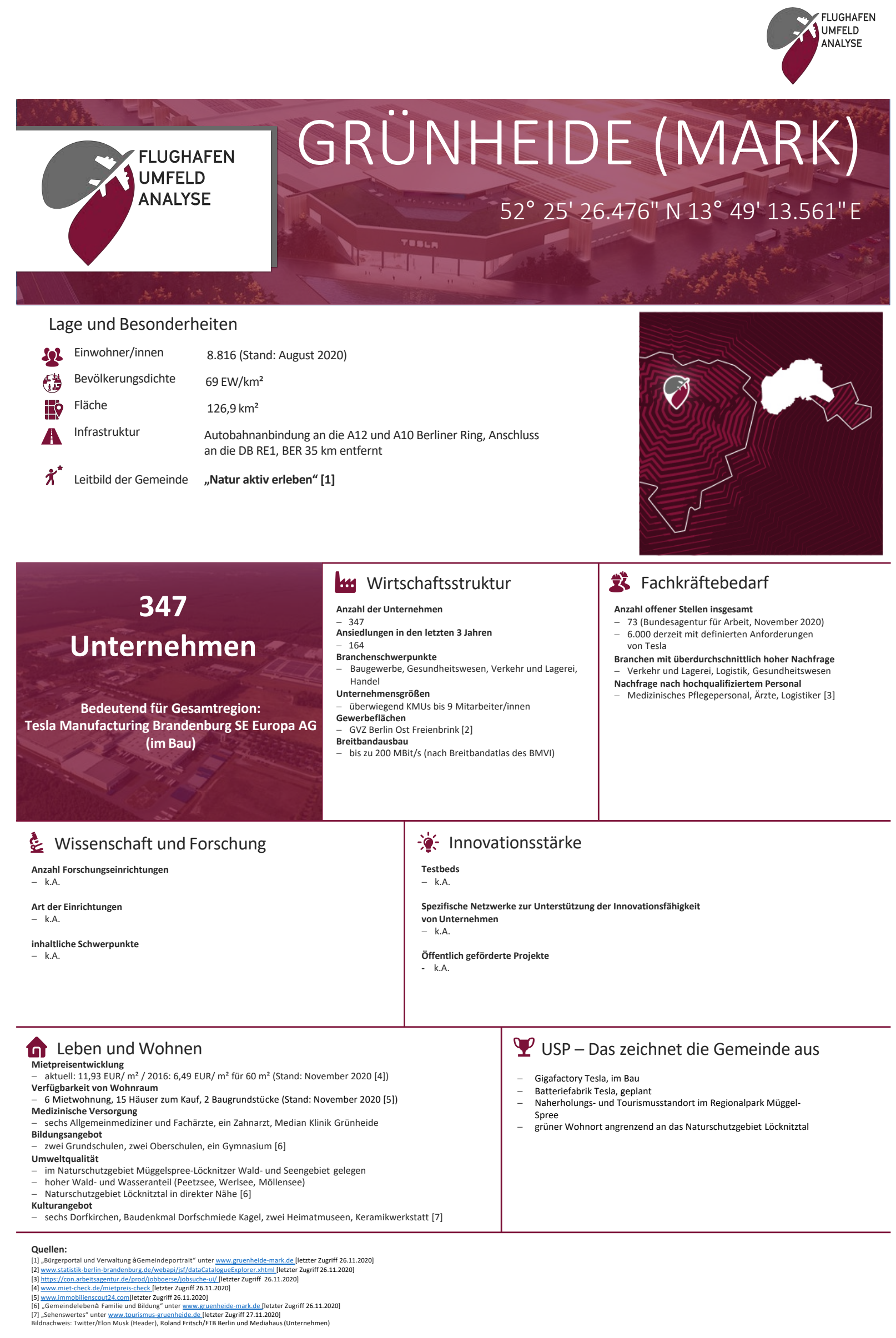




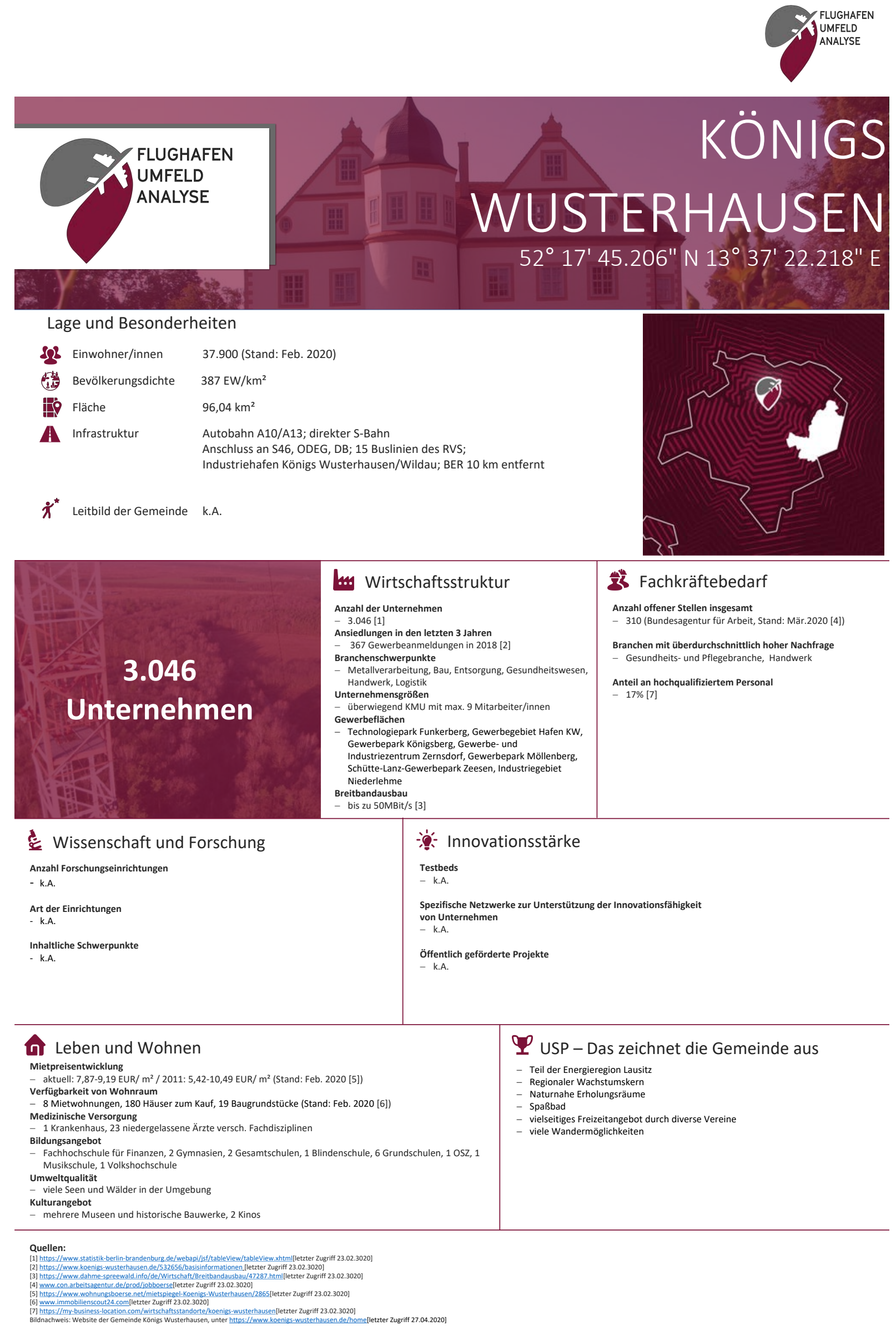




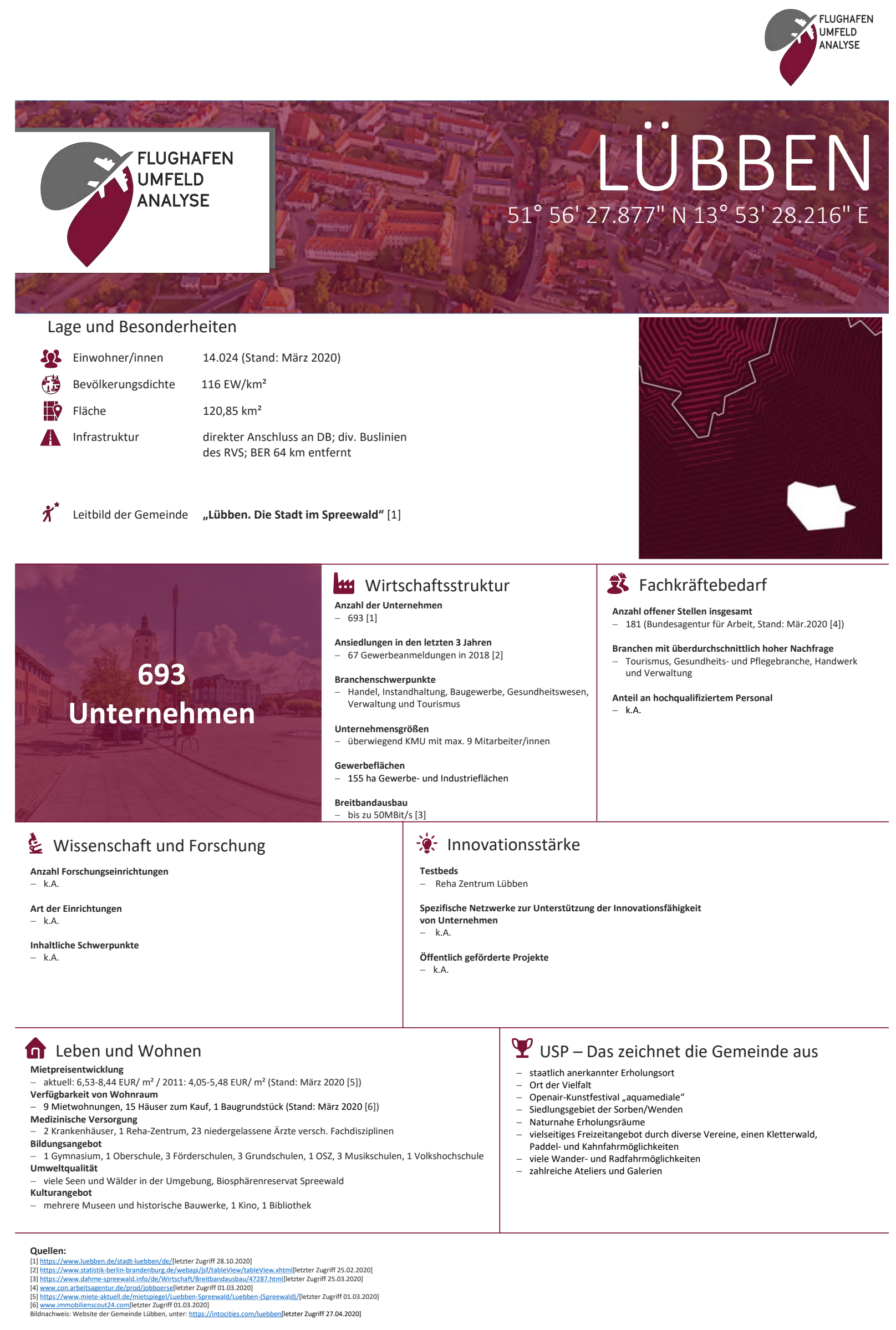




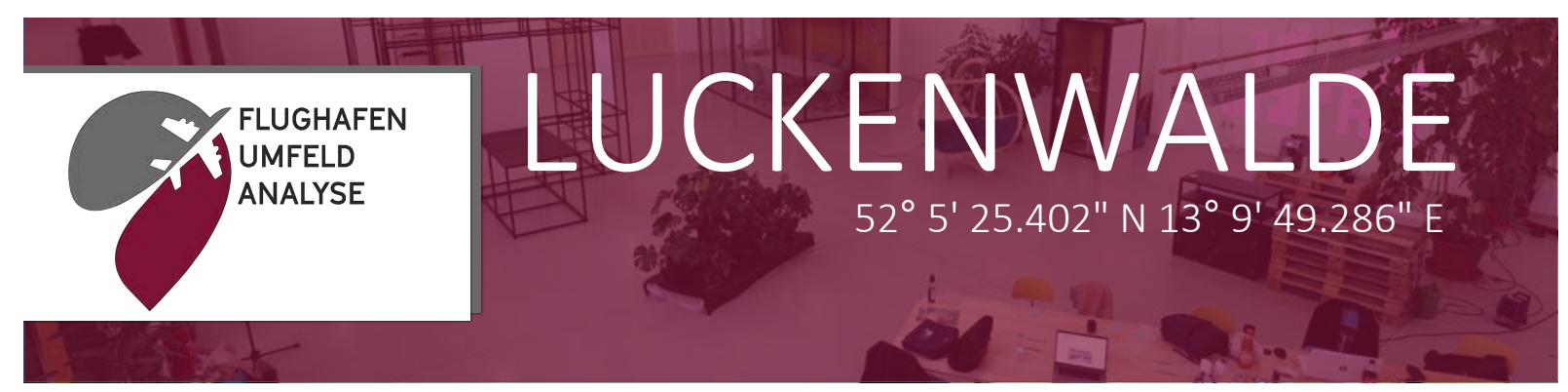

Lage und Besonderheiten
\& Einwohner/innen
21.000 (Stand: Sept. 2020)

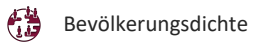
$456 \mathrm{EW} / \mathrm{km}^{2}$
Hë Fläche
$46,64 \mathrm{~km}^{2}$
A Infrastruktur
A10 Berliner Ring: $23 \mathrm{~km}$; direkte Anbindung an die autobahn- ähnlich ausgebaute B 101; ODEG und DB mit den Linien RE3 und RE4; Berlin-Schönefeld künftig BER: 55 km; Berlin-Tegel: 64 km; Verkehrslandeplatz Schönhagen: $17 \mathrm{~km}$
$\AA^{\star}$ Leitbild der Gemeinde „Stadt Luckenwalde: Werkstadt der Moderne - Labor der Zukunft“

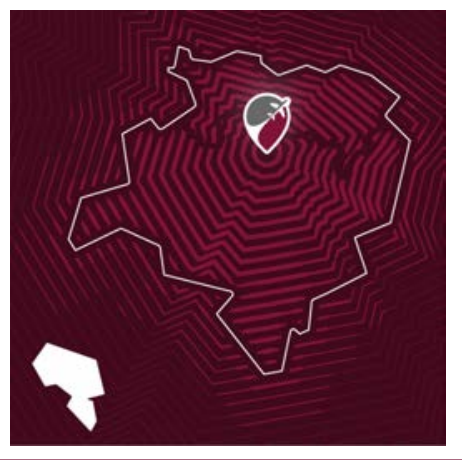

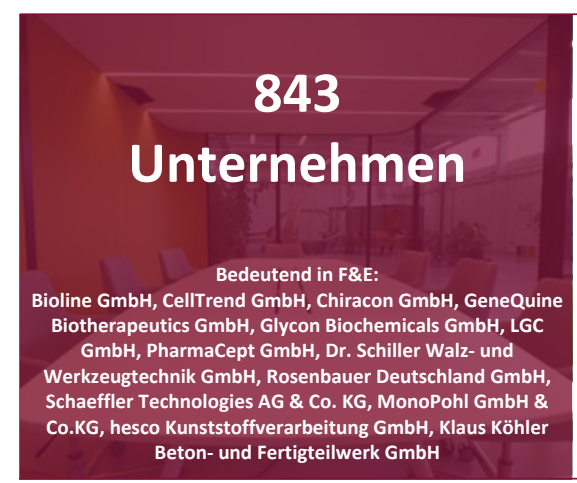

Wissenschaft und Forschung Anzahl Forschungseinrichtungen

Art der Einrichtungen

- Hochschulpräsenzstelle der TH Wildau und der FH Potsdam,

Inhaltliche Schwerpunkte

- Life Science, Digitale Integration und Leichtbau

- Digitale Transformation - Urbane Zukunft

Wirtschaftsstruktur
Anzahl der Unternehmen
-1.874 (Stand: 10/20) [1]
Ansiedlungen in den letzten 3 Jahren
$-\quad 391$ [2]
Branchenschwerpunkte
$-\quad$ Automotive, Metall, Life Sciences, Gesundheitswirtschaft,
Biotechnologie, Mikroelektronik
Unternehmensgrößen
$-\quad$ überwiegend KMU mit max. 49 Mitarbeiter/innen
Gewerbeflächen
- Industrie- und Gewerbegebiet Zapfholzweg,
Industriegebiet Luckenwalde, Gewerbegebiet Am
Honigberg, Gewerbegebiet Berkenbrücker Chaussee,
Gewerbegebiet Am Frankenfelder Berg,
Biotechnologiepark Luckenwalde
Breitbandausbau
- bis zu 400 MBit/s [3]

כ3ॅ Fachkräftebedarf

Anzahl offener Stellen insgesamt

- ca. 200 (Bundesagentur für Arbeit, Stand: Feb.2020 [4])

Branchen mit überdurchschnittlich hoher Nachfrage

- Biotechnologie, Life Sciences und Metall[1]

Nachfrage nach hochqualifiziertem Personal - k.A.
:- Innovationsstärke

Testbeds

- Coworkingspace, Showroom und Makerspace der Präsenzstelle

Spezifische Netzwerke zur Unterstützung der Innovationsfähigkeit

von Unternehmen

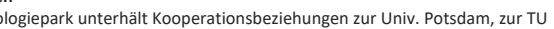

Berlin, zur FU Berlin und zur TH Wildau [6]

Öffentlich geförderte Projekte

Präsenzstelle der TH Wildau und der FH Potsdam

Landkenzstelle der TH Wildau und der FH Potsdam
Landender Ausbau und Vervollständigung der Fahrradinfrastruktur sowie

Einrichtung eines Fahrradverleihsystems zwischen Nuthe und Nieplitz (BMU) [8]

KSI: Energiesparmodell für vier Luckenwalder Schulen (BMU) [8]

\begin{tabular}{|c|c|}
\hline $\begin{array}{l}\text { Leben und Wohnen } \\
\text { Mietpreisentwicklung } \\
- \text { aktuell: } 6,52 \mathrm{EUR} / \mathrm{m}^{2} / 2011: 4,91 \mathrm{EUR} / \mathrm{m}^{2} \text { für } 60 \mathrm{~m}^{2} \text { (Stand: Feb. } 2020 \text { [7]) } \\
\text { Verfügbarkeit von Wohnraum } \\
-23 \text { Mietwohnungen, } 25 \text { Häuser zum Kauf, } 5 \text { Baugrundstücke (Stand: Feb. } 2020[8] \text { ) } \\
\text { Medizinische Versorgung } \\
-\quad \text { Krankenhaus mit } 15 \text { Fachabteilungen [9]; niedergelassene Ärzte sämtlicher Fachdisziplinen; Medizinisches } \\
\text { Versorgungszentrum und Hospitz } \\
\text { Bildungsangebot } \\
-\quad \text { drei Grundschulen, eine Oberschule, ein Gymnasium, Oberstufenzentrum, Kreismusikschule, } \\
\text { Volkshochschule, Schule für Gesundheitsfachberufe am KMG Klinikum Luckenwalde, mehrere private } \\
\text { Bildungsträger } \\
\text { Umweltqualität } \\
\text { - umgeben von Wäldern und Fluren; naturnahe Erholungsgebiete sowie Frischluftschneisen } \\
\text { Kulturangebot } \\
-\quad \text { Kino, Theater, Bibliothek, verschiedene Kultur-Cafés, aktive Künstlerszene, vielfältige Veranstaltungsformate }\end{array}$ & $\begin{array}{l}1 \text { USP - Das zeichnet die Gemeinde auS } \\
\text { - Kreisstadt des Landkreises Teltow-Fläming } \\
\text { - Mittelzentrum und Regionaler Wachstumskern im Land Brandenburg } \\
\text { - sehr schnelle Verkehrsanbindung an Berlin über Schiene und Straße (oder: schnelle } \\
\text { Verbindung in die Hauptstadt und zum BER) } \\
\text { - kurze Wege innerhalb der Stadt } \\
\text { - Biotechnologiepark und Platz für Neuansiedlungen } \\
\text { - Hochschulpräsenzstelle der TH Wildau und FH Potsdam } \\
\text { - hervorragendes Netzwerk aus Unternehmen, Institutionen, Behörden und } \\
\text { Verbänden } \\
\text { - besondere Wohnstandorte im Gründerzeitquartier und in sanierten Fabrikgebäuden } \\
\text { - zahlreiche Freizeit- und Kulturangebote: Freizeitbad, Flaeming-Skate, Stadion, } \\
\text { Tierpark, Theater, Kino, Bibliothek im Bahnhof } \\
\text { - naturnahe Erholungsräume } \\
\text { - städtische KMU-Förderung für Unternehmen }\end{array}$ \\
\hline 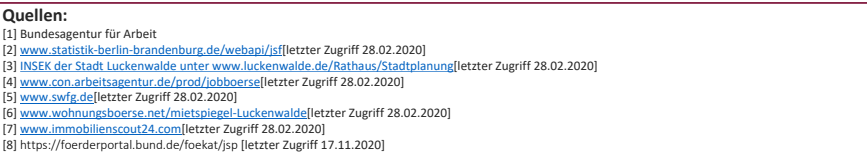 & \\
\hline
\end{tabular}




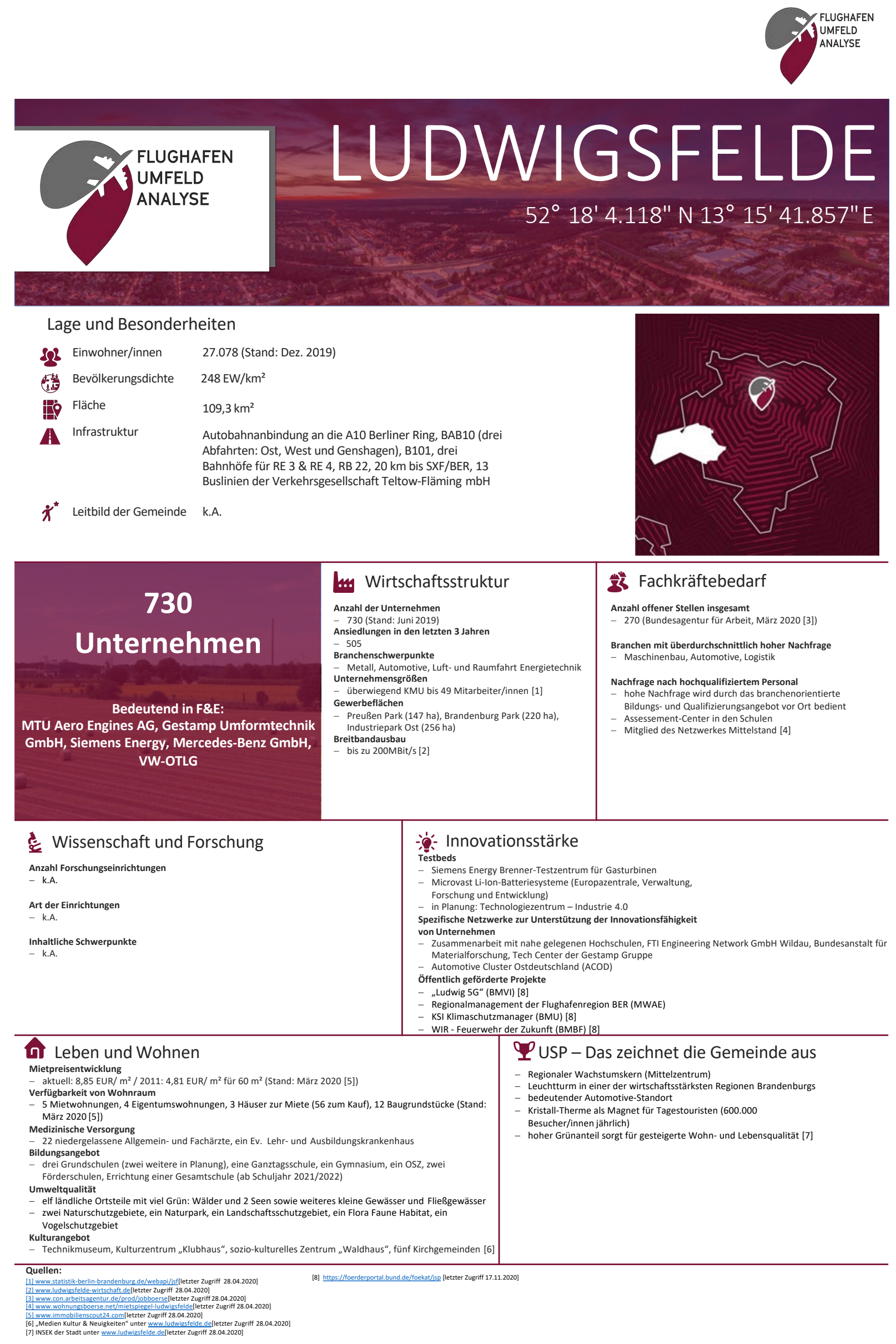




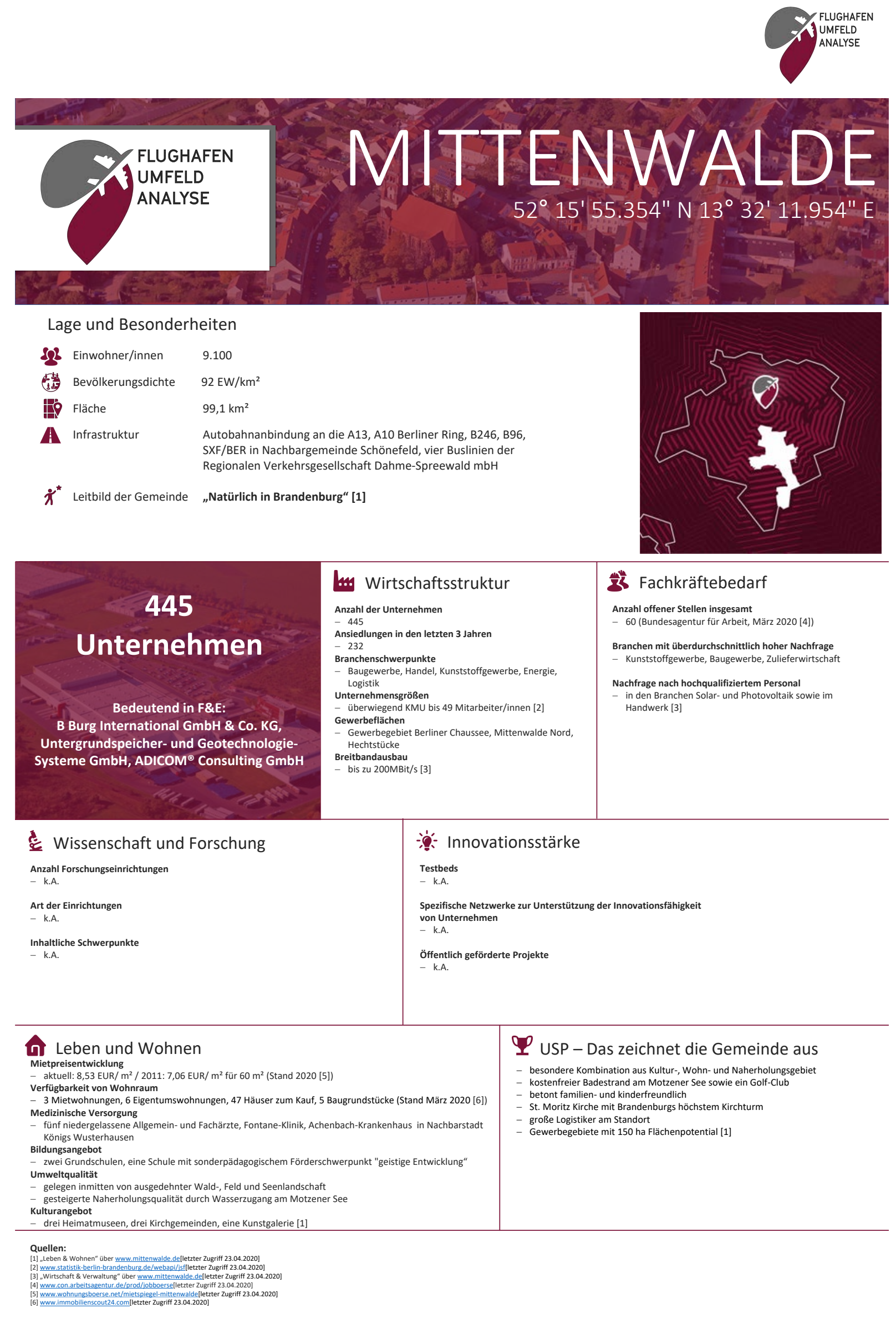




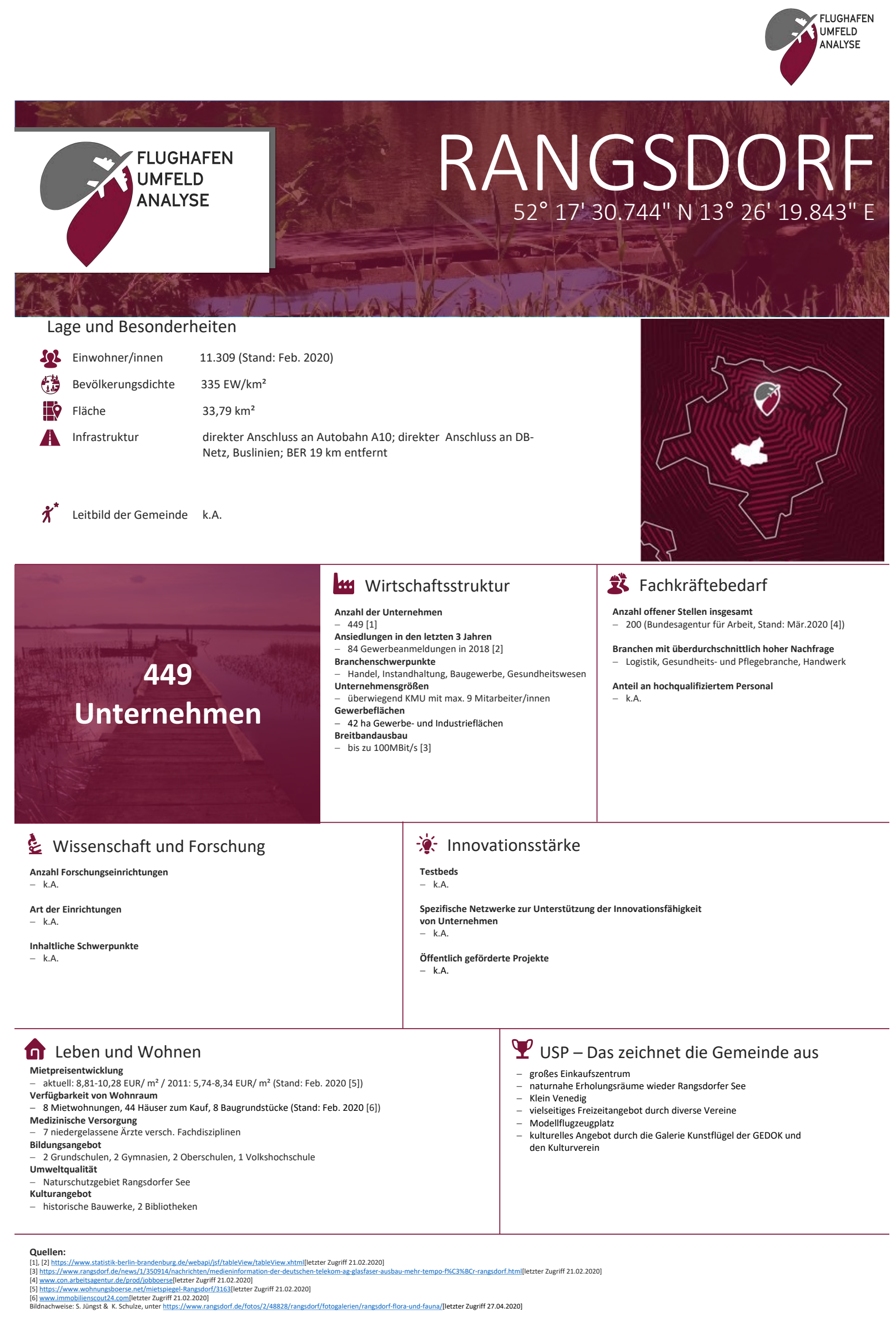




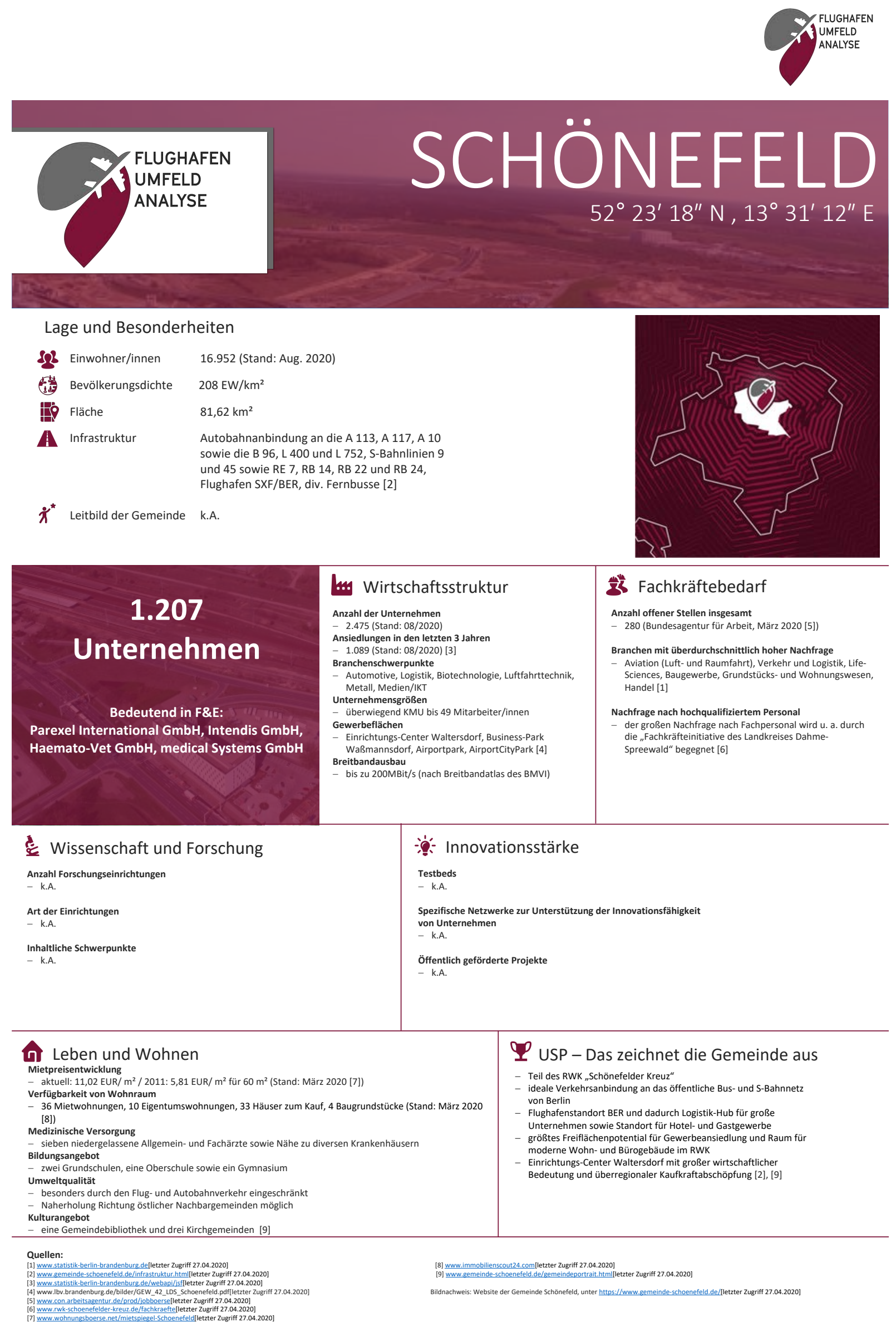




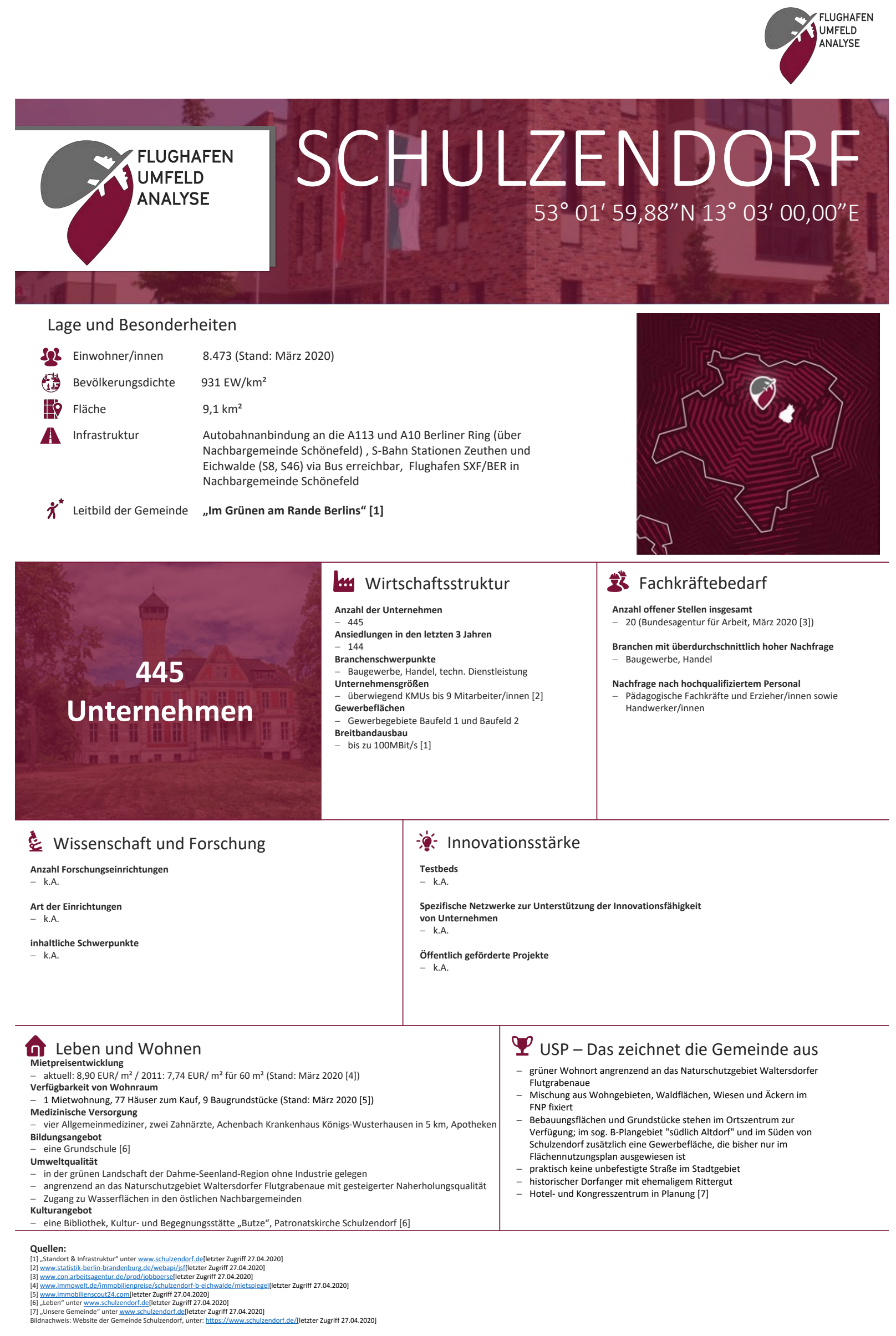




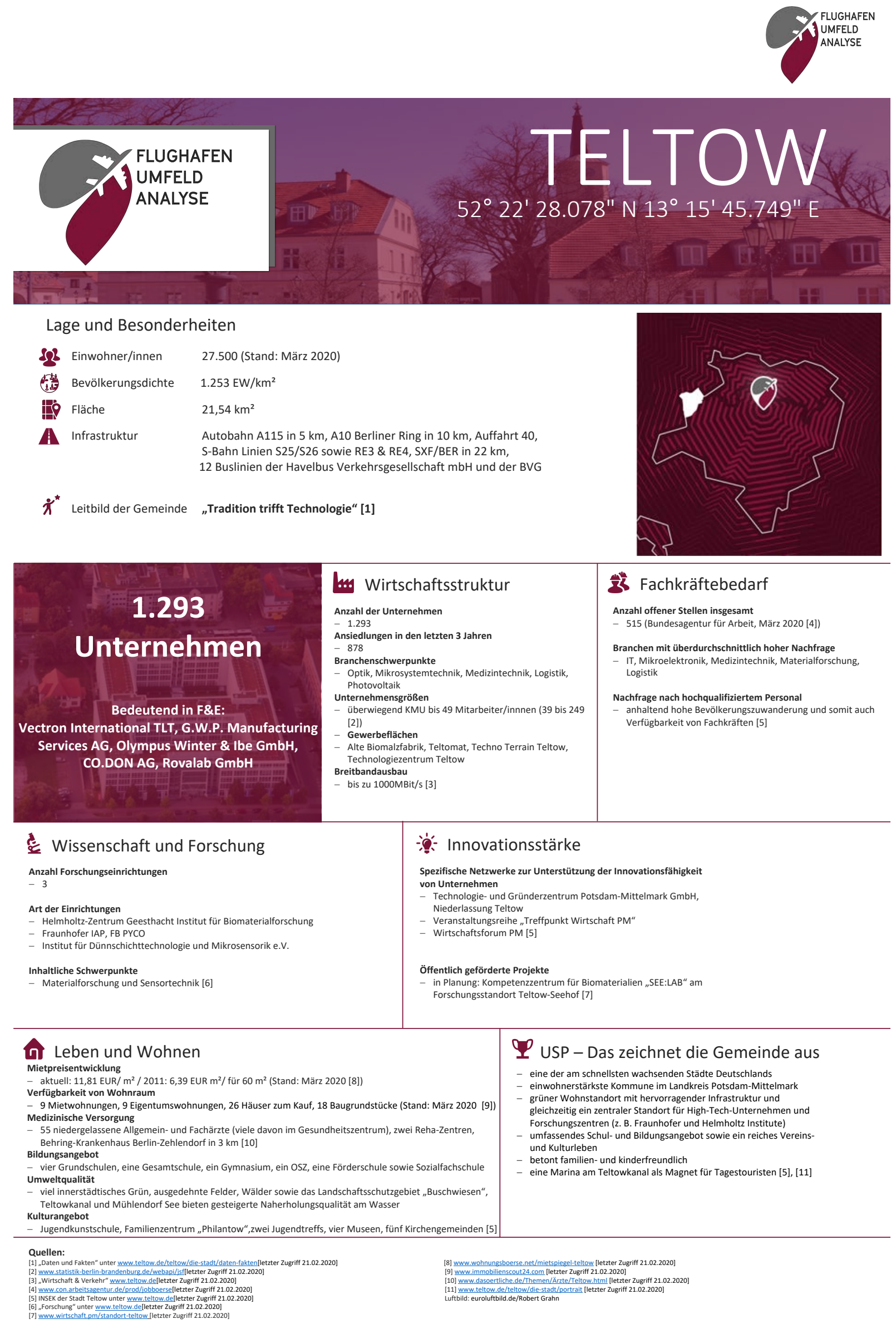




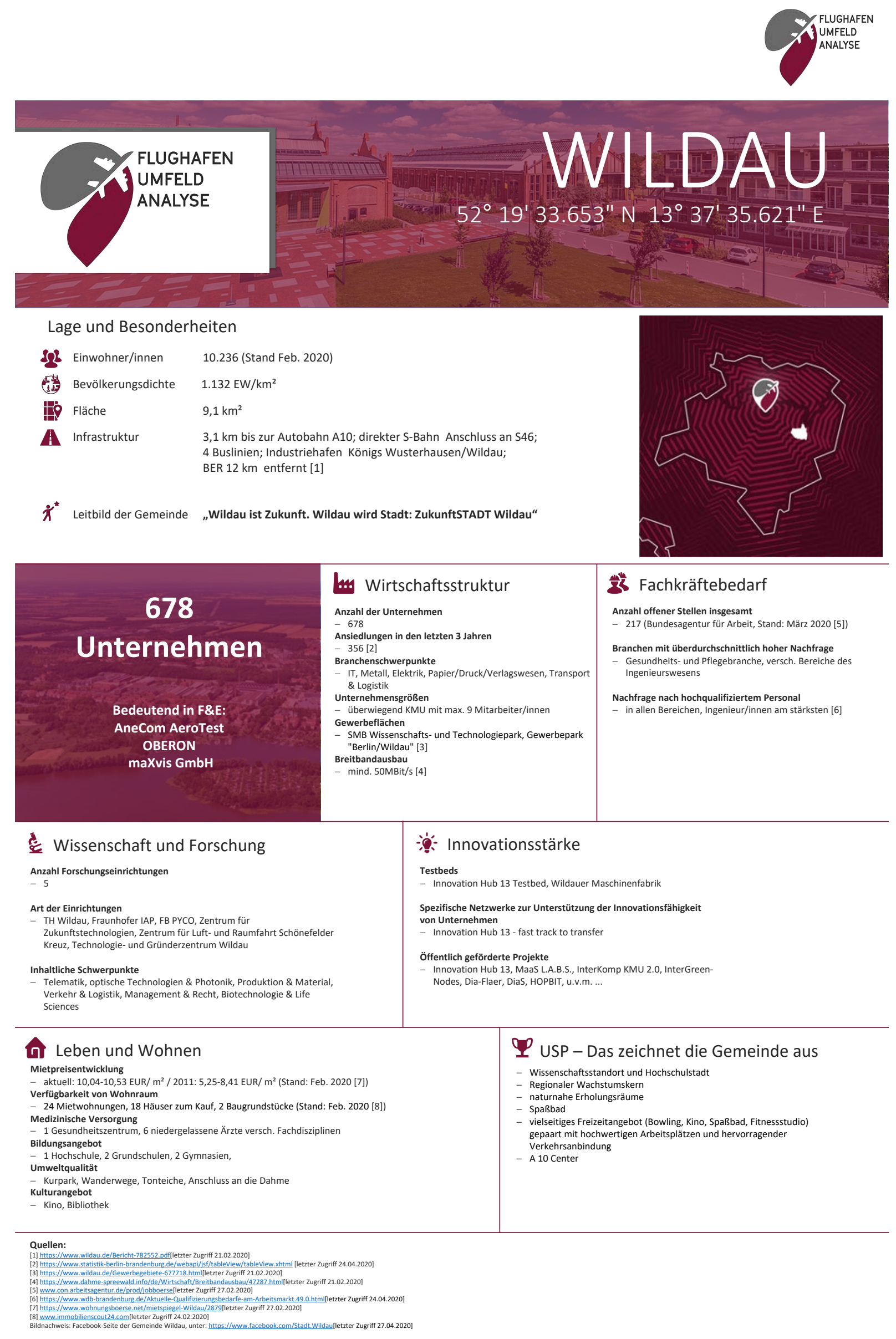




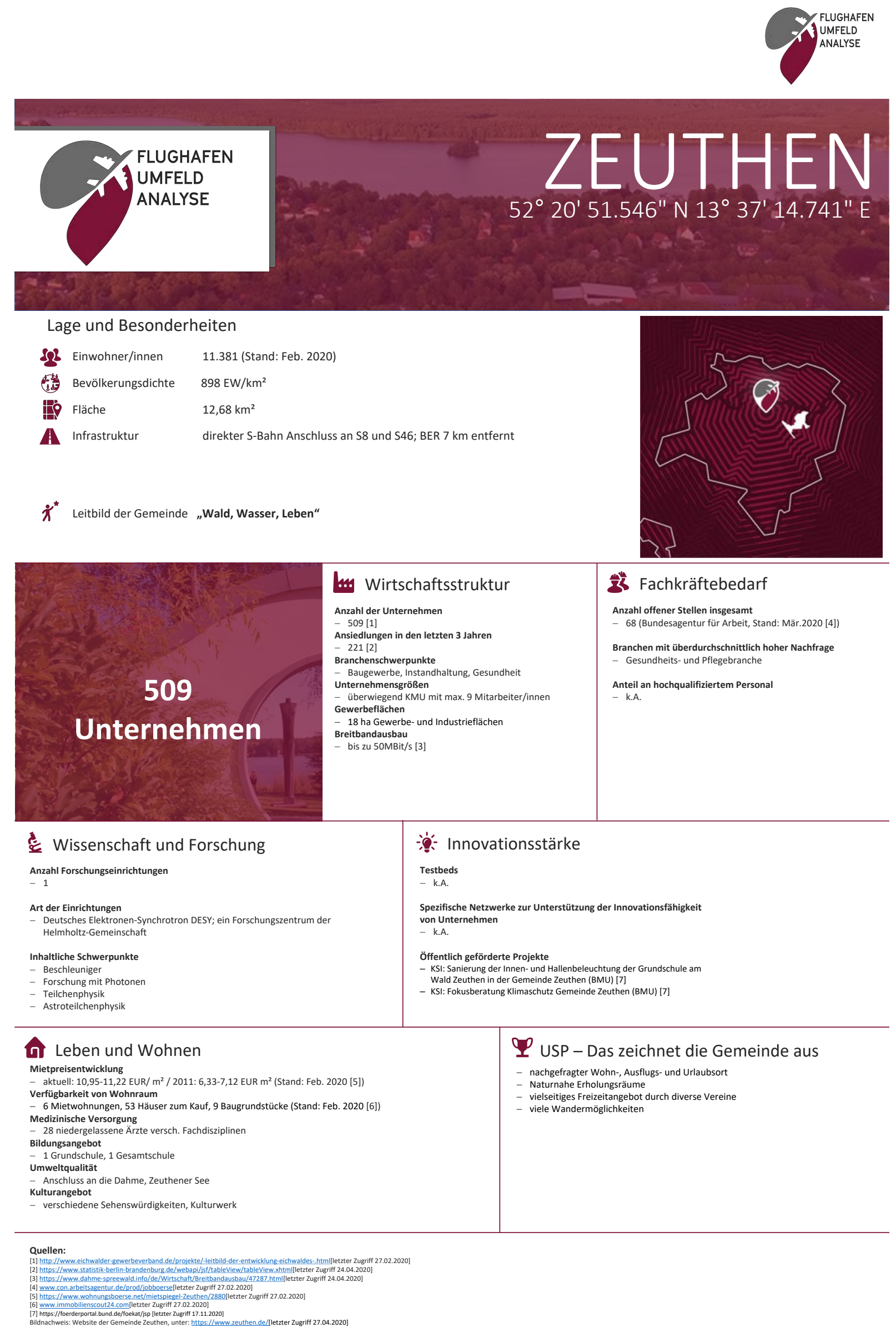




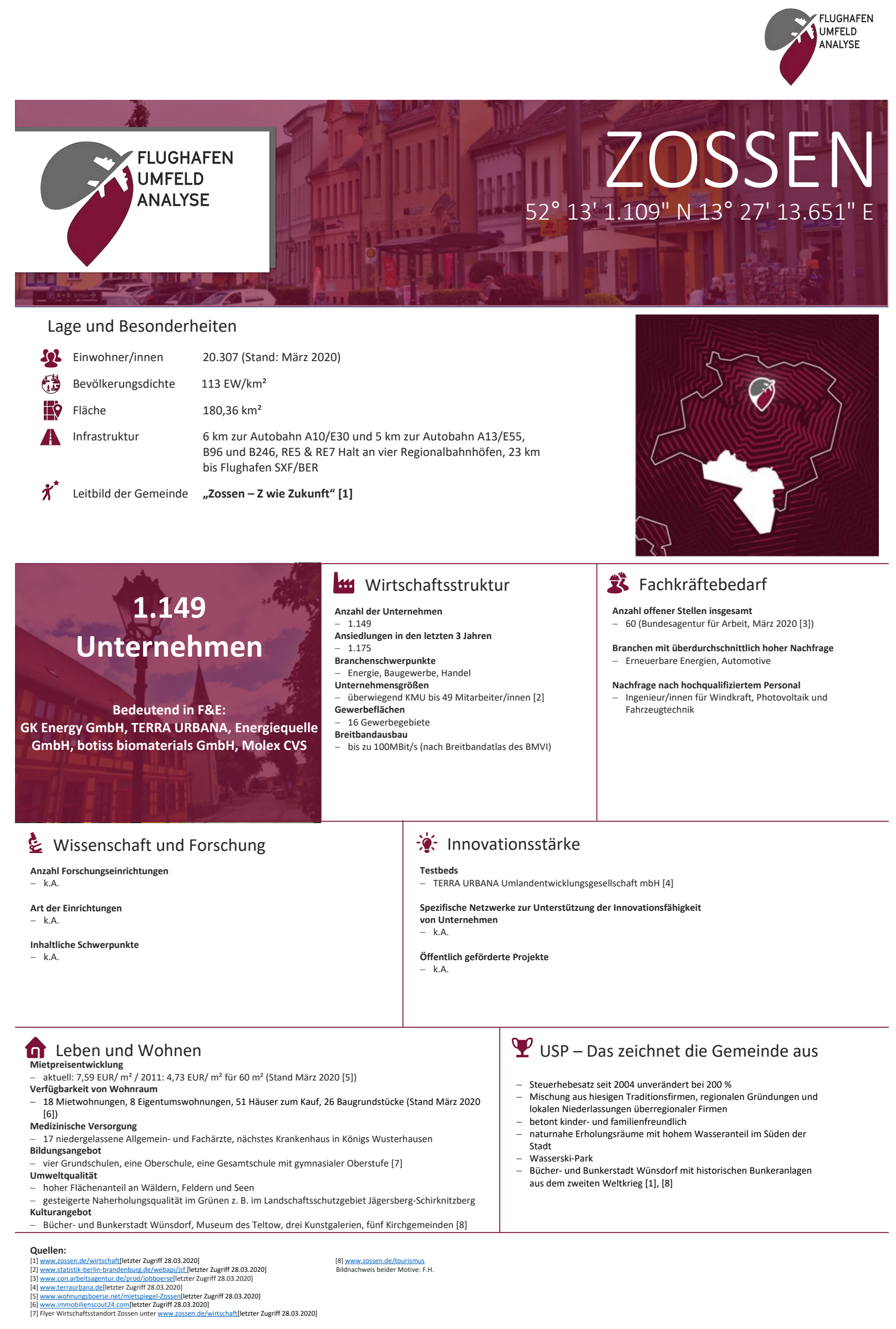




\section{Anlage 2 Good Practice Beispiele}

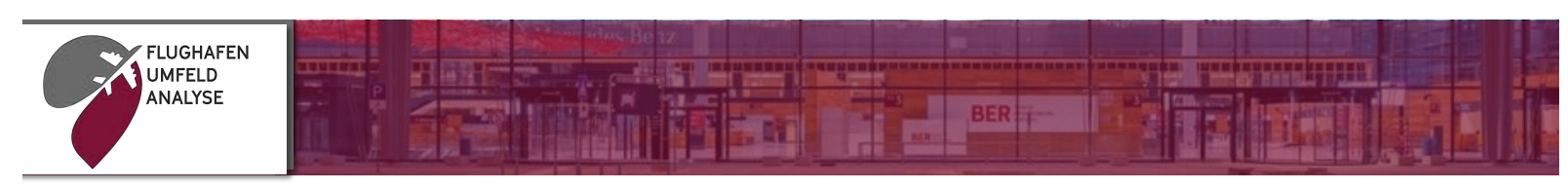

Good Practice Beispiele

Themenfelder und Flughäfen/Flughafenregionen

\begin{tabular}{|l|l|}
\hline Themenfeld & Flughafen/Flughafenregion \\
\hline 1. Governance & $\begin{array}{l}\text { Stockholm - Arlanda Airport } \\
\text { Atlanta - Hartsfield Jackson International Airport }\end{array}$ \\
\hline 2. Ansiedlung & $\begin{array}{l}\text { Atlanta - Hartsfield Jackson International Airport } \\
\text { München - Flughafen München }\end{array}$ \\
\hline 3. Mobilität & $\begin{array}{l}\text { Stockholm - Arlanda Airport } \\
\text { Hong Kong - International Airport }\end{array}$ \\
\hline 4. Fachkräfte & $\begin{array}{l}\text { Helsinki - Aviapolis } \\
\text { Singapore - Changi Airport }\end{array}$ \\
\hline 5. Marketing & Seoul - Incheon Airport \\
\hline
\end{tabular}

Hauptquelle: IAU île-de-France, 2018. Sustainable Airport Areas. Guidelines for Decision Makers. [Onlinel.

Verfügbar unter: https://www.metropolis.org/sites/defautl/files/resources/2018.03 Sustainable Airport Areas Guidelines for decision makers 0.pdf.
[letzter Zugriff: 27.112020$]$.

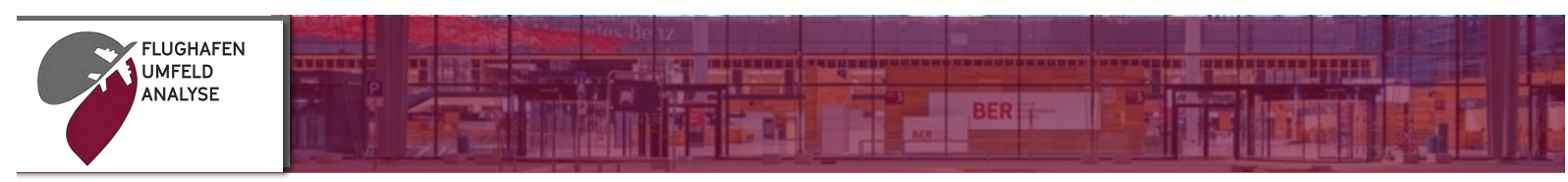

Good Practice Beispiel im Bereich Governance

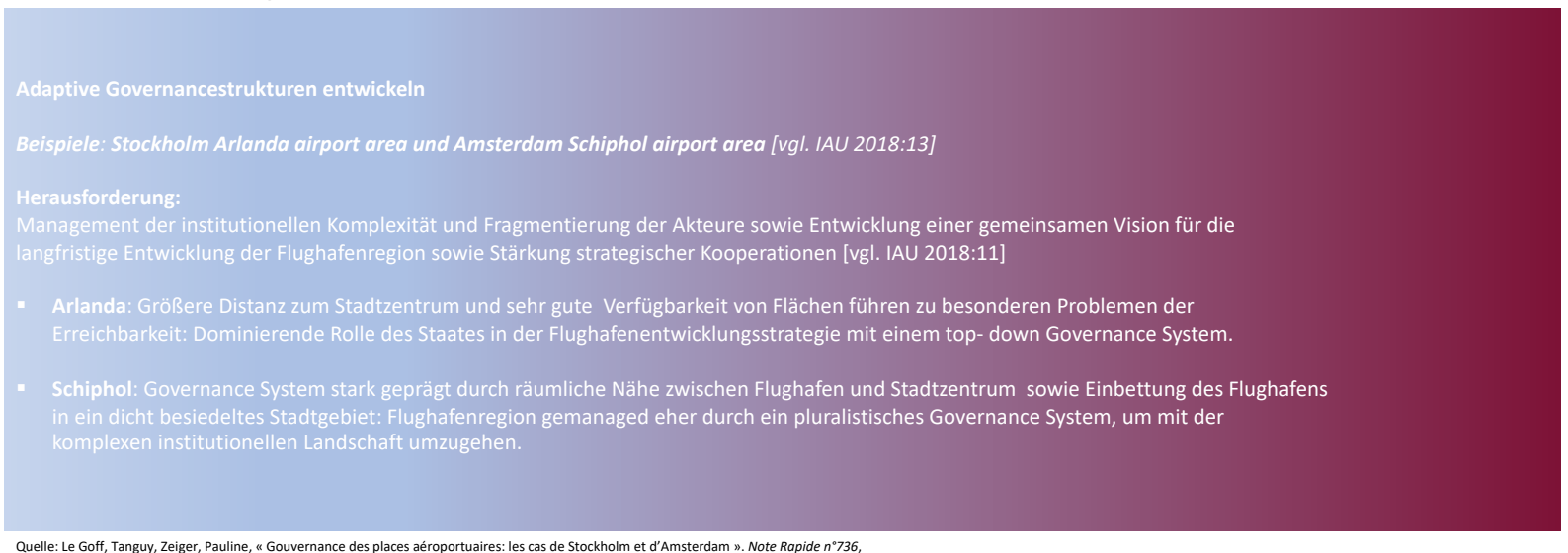

Quelle: Le Goff, Tanguy, Zeiger, Pauline, " Gouvernance des places aéroportuaires: les cas de Stockholm et d'Amsterdam ». Note Rapide n 736 ,

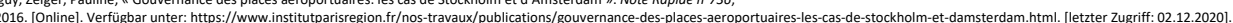




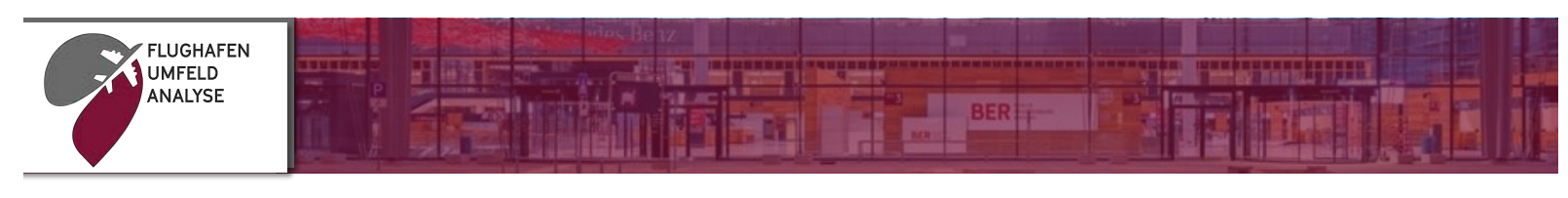

Good Practice Beispiel im Bereich Governance

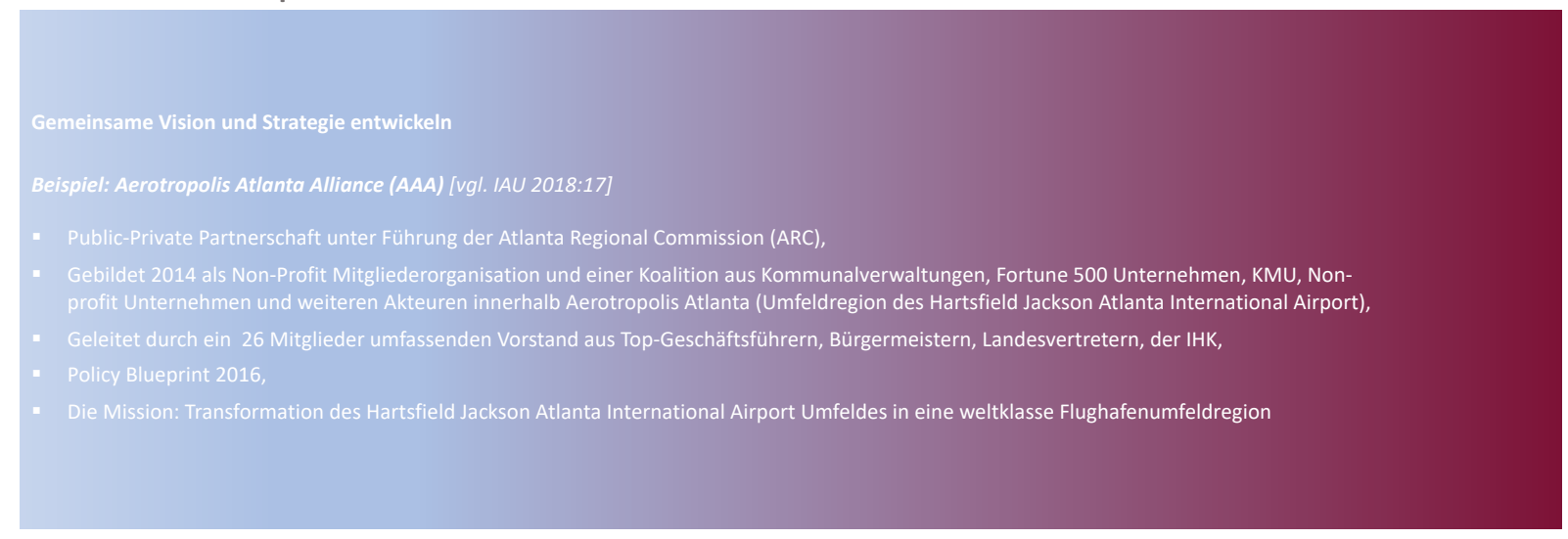

Quellen: [Online]. Verfügbar unter: hittps://aeroatl.org/. [letzter Zugriff: 07.12.2020] und https://aeroatl.org/wp-content/uploads/2017/06/aerotropolis-atlanta-blueprint-final.pdf. [letzter Zugriff. 07.12.2020].

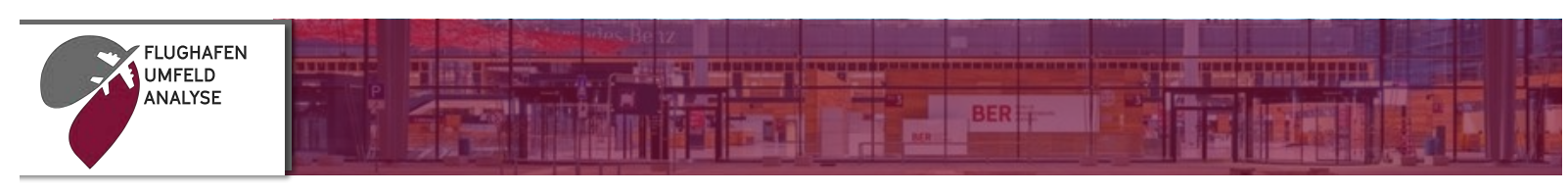

Good Practice Beispiel im Bereich Ansiedlung

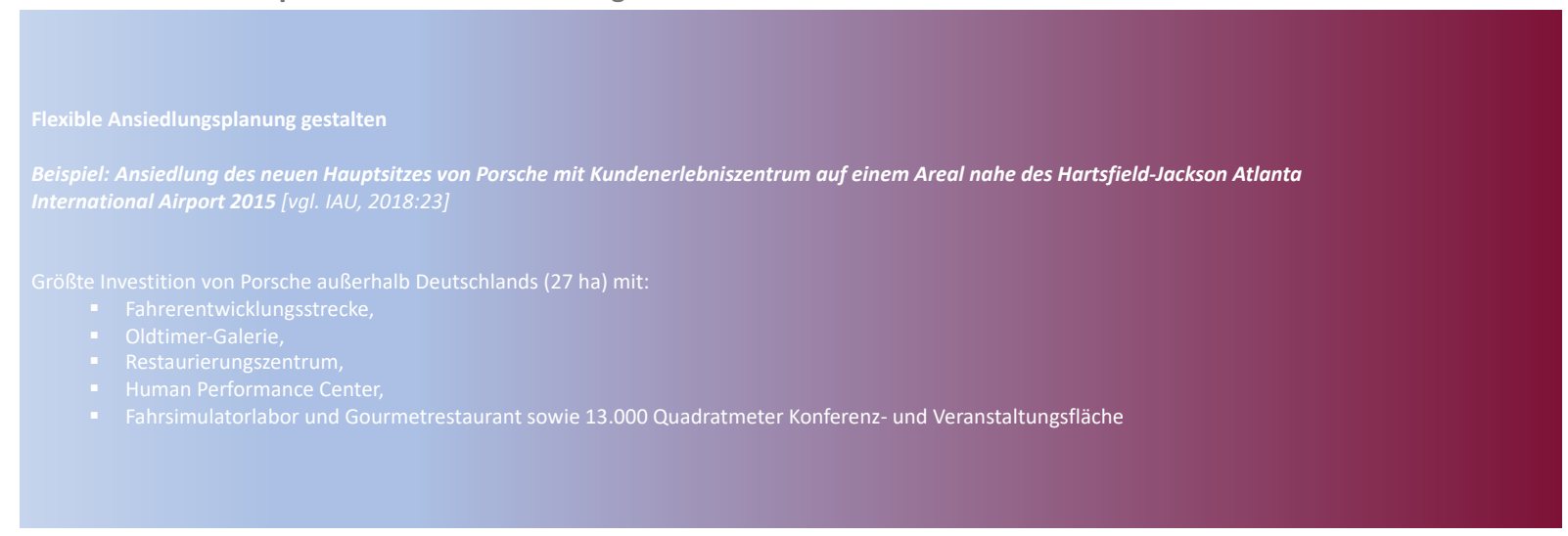

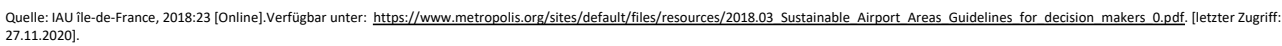




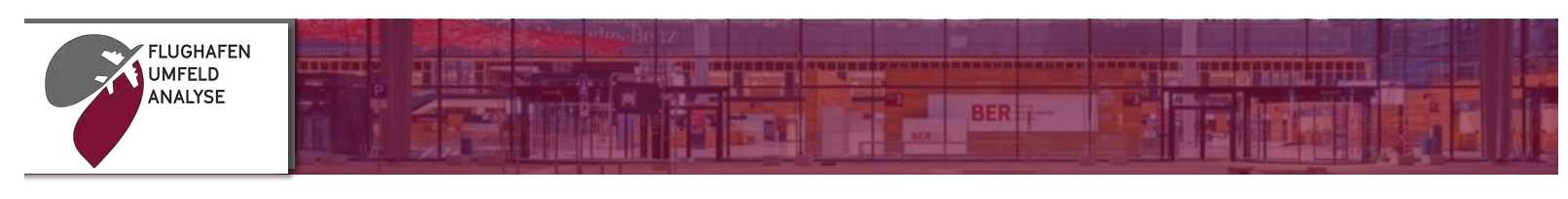

Good Practice Beispiel im Bereich Ansiedlung

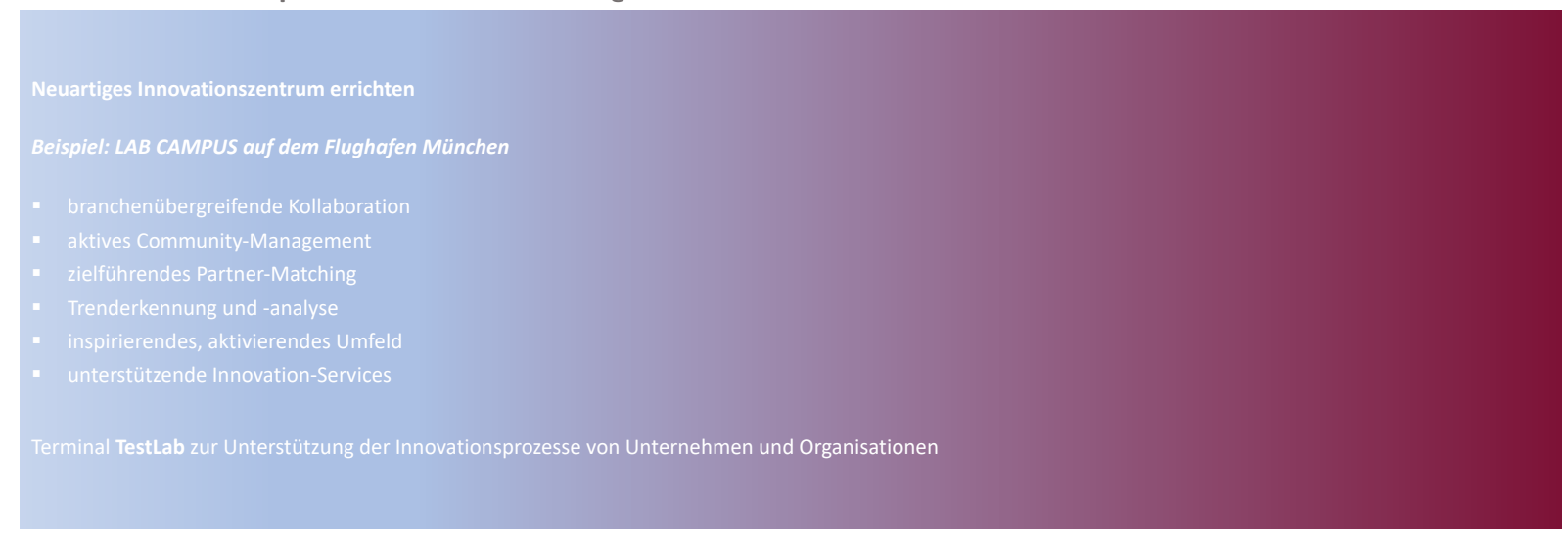

Quelle: [Online]. Verfübar unter: https://www.labcampus.de/de/innovation [Zugriff: 27.11.2020].

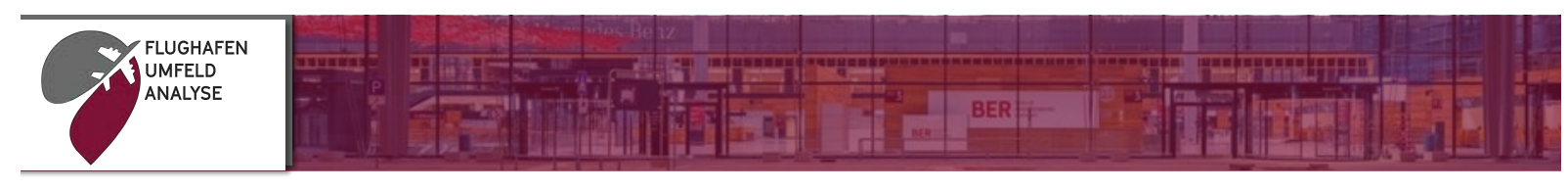

Good Practice Beispiel im Bereich Mobilität

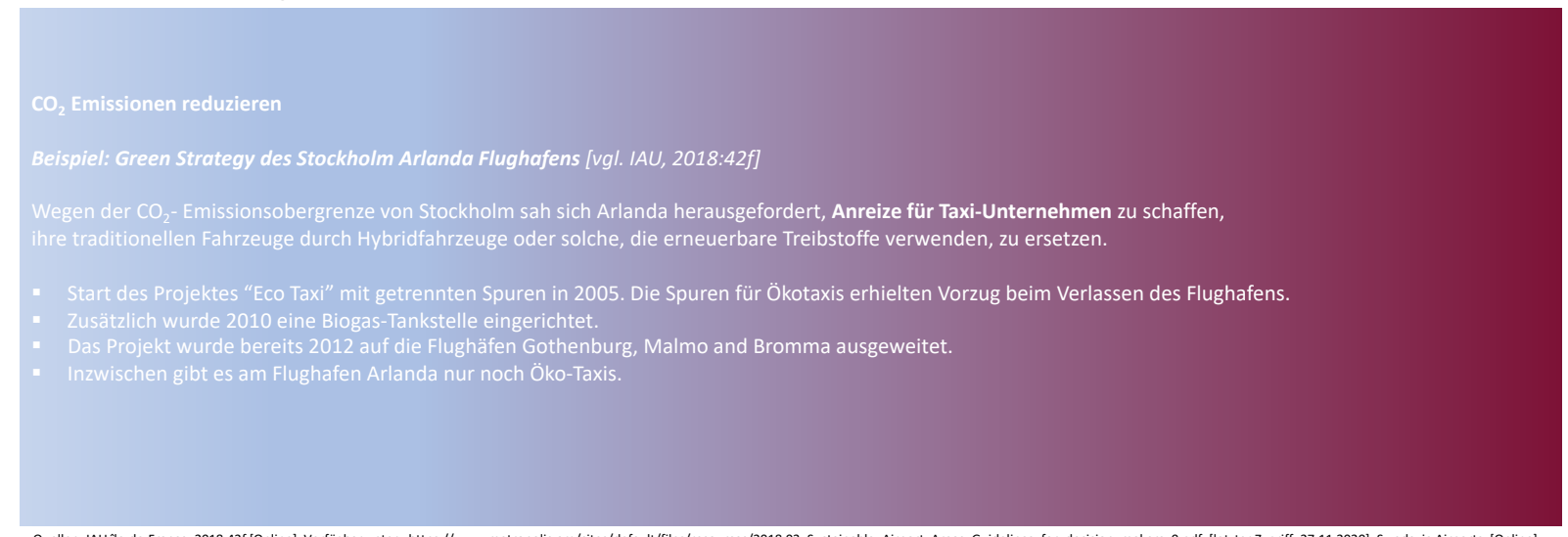

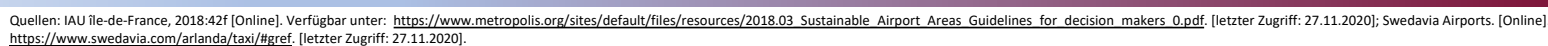




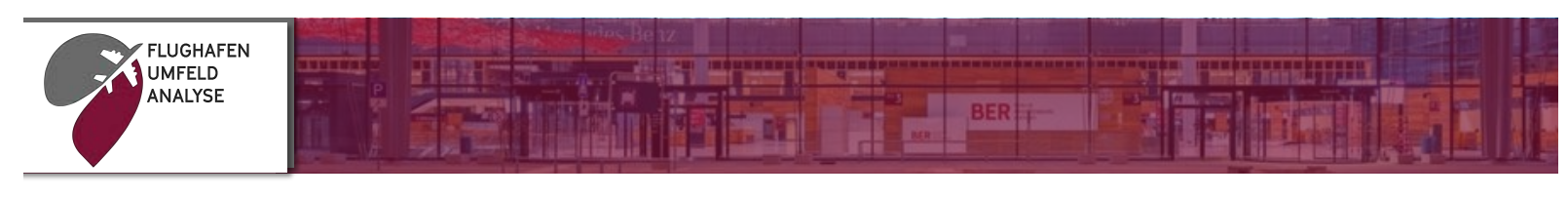

Good Practice Beispiel im Bereich Mobilität

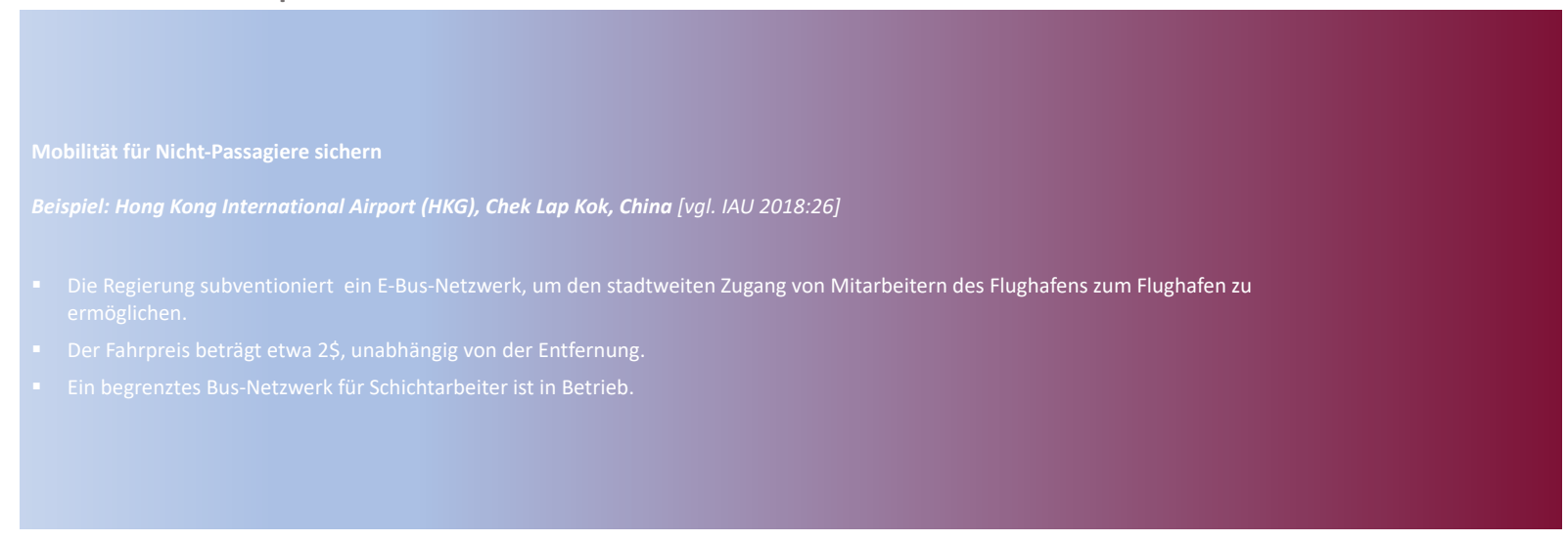

Quelle: [Online]. Verfügbar unter: https://www.legco.gov.hk/yr16-17/english/hc/sub_com/hs102/paper/hs10220170207cb4-491-2-e.pdf. [letzter Zugriff: 02.12.2020].

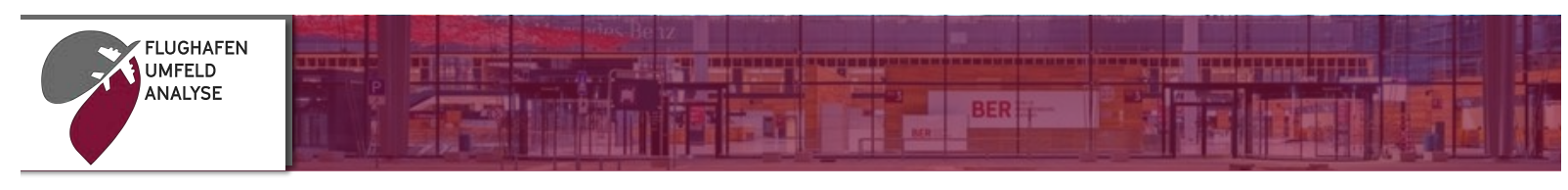

Good Practice Beispiel im Bereich Fachkräfte

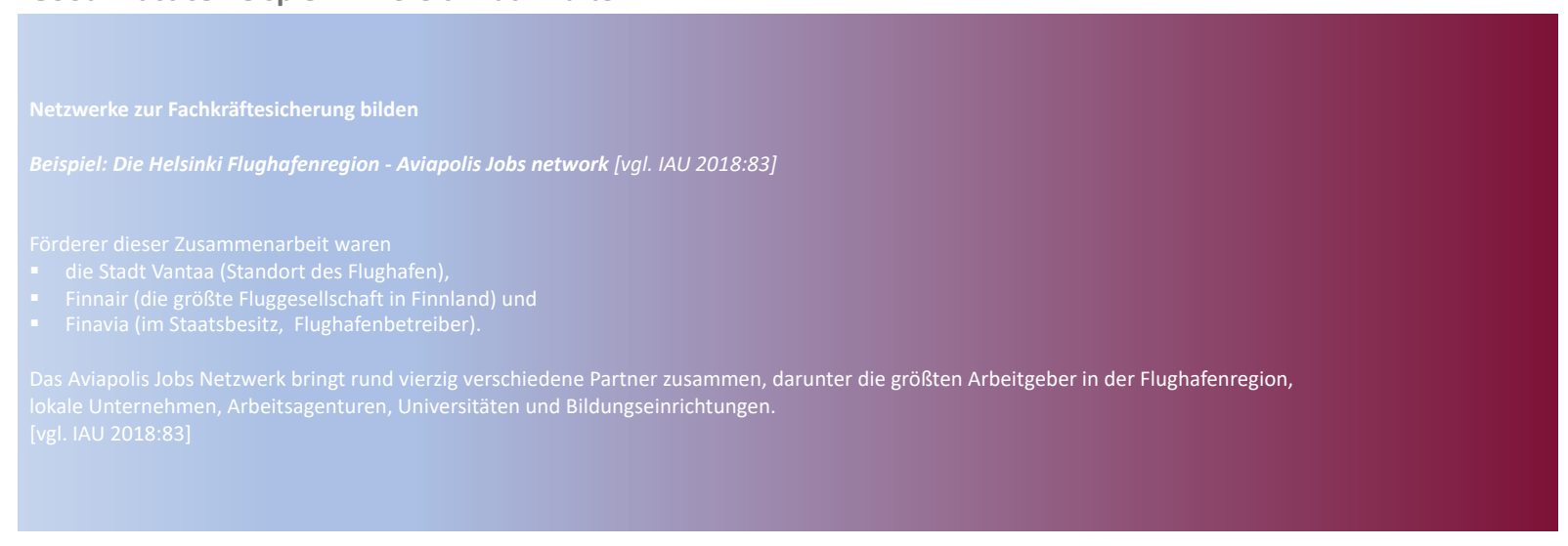

Quelle: [Online]. Verfügbar unter: https://translate. Boogle.com/translate?hl=de\&sl=auto\&tl=de\&u=http\%3A\%2F\%2Ftyopaikat.aviapolis.fi\%2F. [letzter Zugriff: 07.12.2020]. 


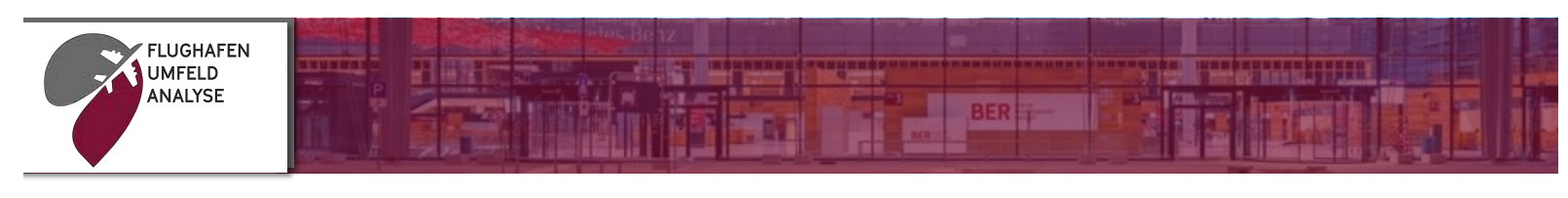

Good Practice Beispiel im Bereich Fachkräfte

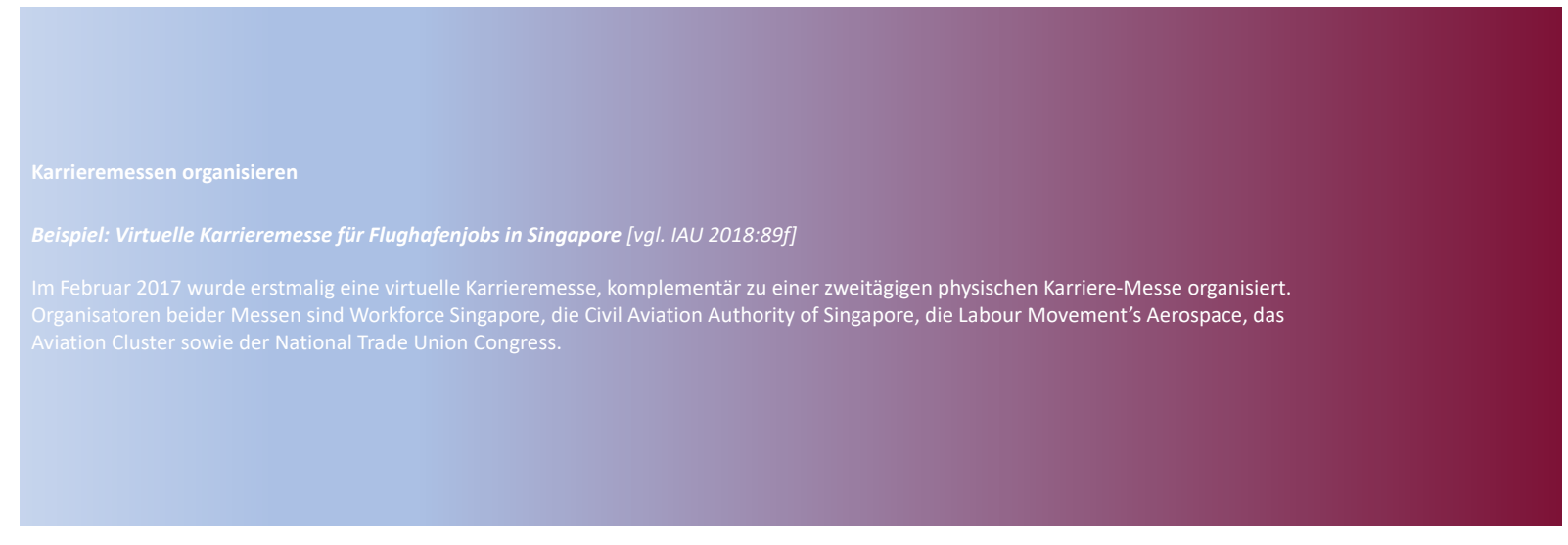

Quelle: [Online]. Verfügbar unter: https://vcf.mycareersfuture.sg/vcf. [letzter Zugriff:07.12.2020].

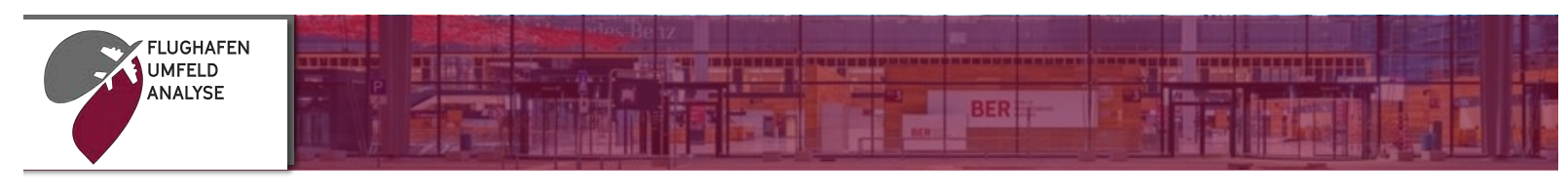

Good Practice Beispiel im Bereich Marketing

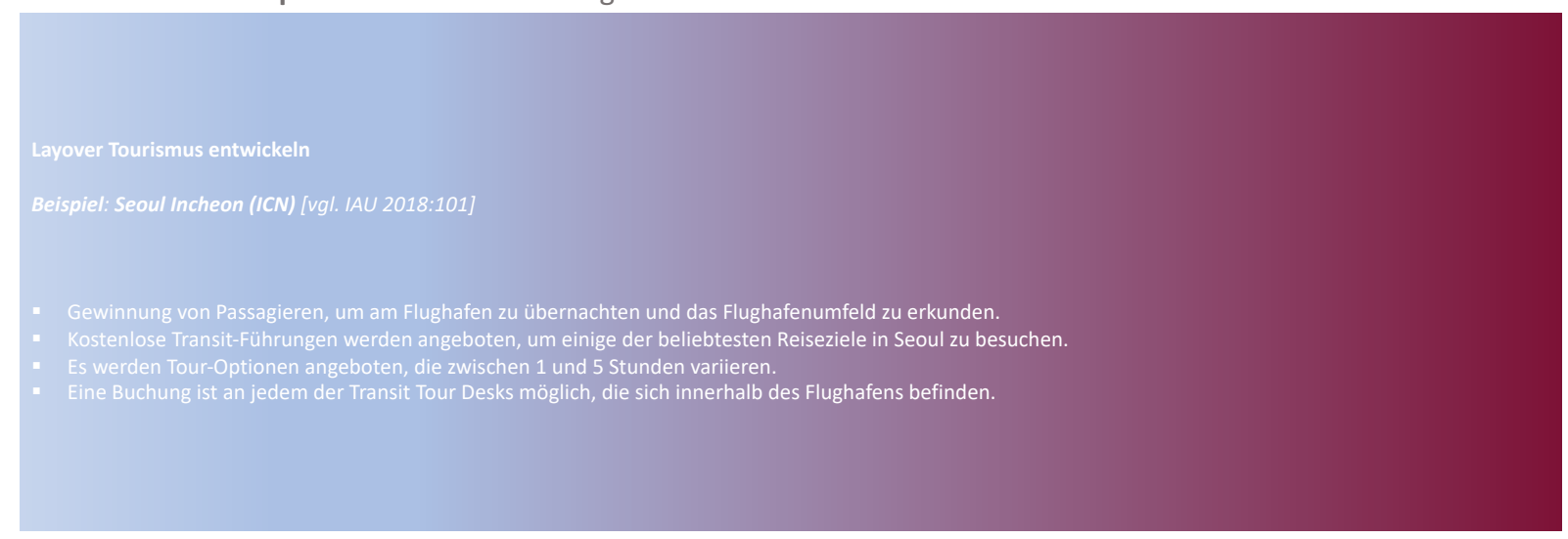

Quelle: [Online]. Verfügbar unter: https://www.airport.kr/ap cnt/en/trn/tour/trcose/trcose.do. [letzter Zugriff: 07.12.2020]. 


\section{Anlage 3 Programm Digitales Forum}

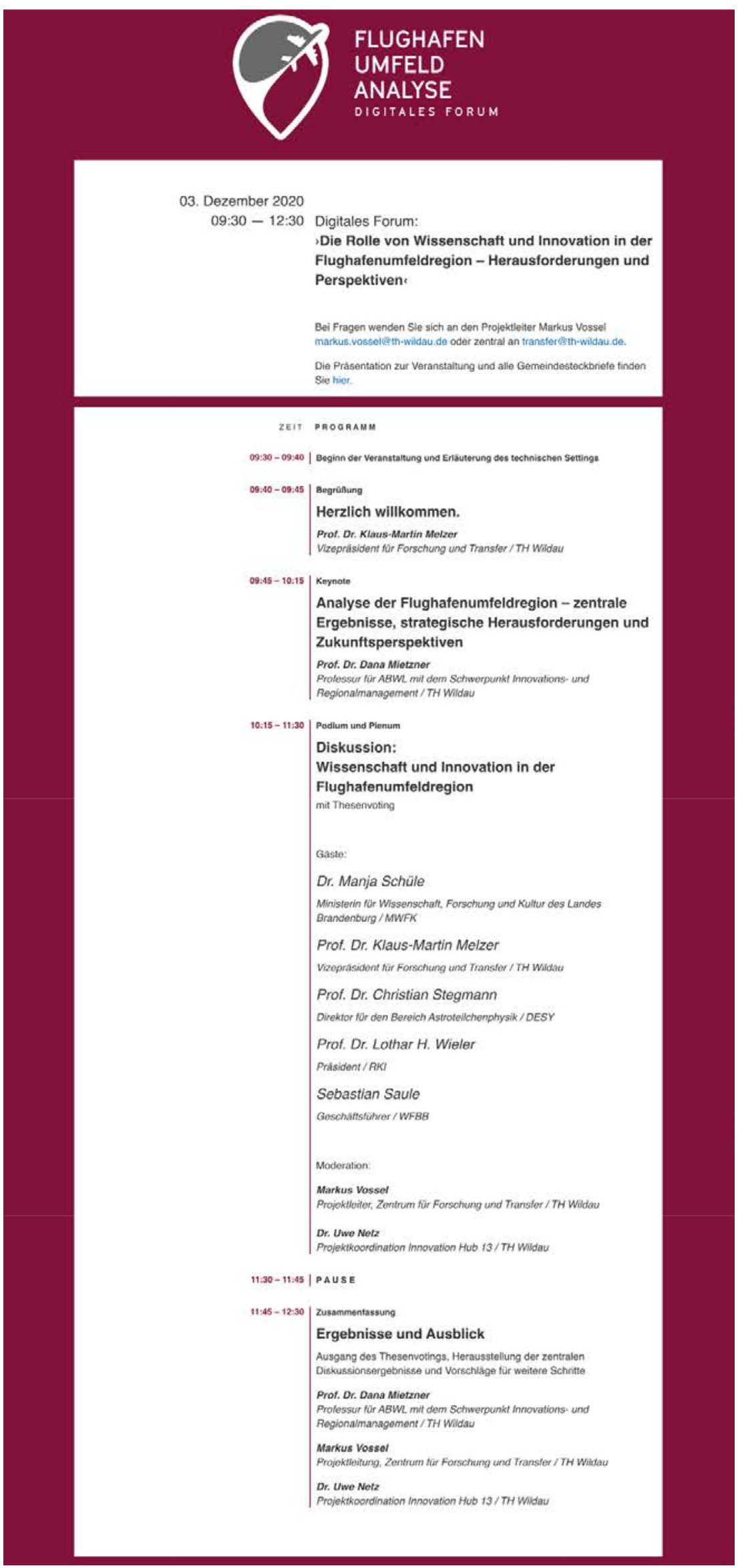

